Supporting information for:

\title{
Fall Creek Monitoring Station: using environmental covariates to predict micropollutant dynamics and peak events in surface water systems
}

Corey M. G. Carpenter ${ }^{1}$, Lok Yee J. Wong ${ }^{2}$, Danyeh L. Gutema ${ }^{1}$, and Damian E. Helbling ${ }^{\text {I* }}$

${ }^{1}$ School of Civil and Environmental Engineering, Cornell University, Ithaca, NY, USA

${ }^{2}$ Department of Biological and Environmental Engineering, Cornell University, Ithaca, NY, USA

*Corresponding author: damian.helbling @ cornell.edu, phone: +1 607255 5146, fax: +1 607255 9004

\# of pages: 51

\# of tables: 3

\# of figures: 65 


\section{Table of Contents}

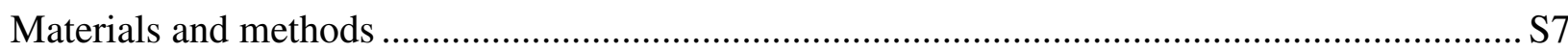

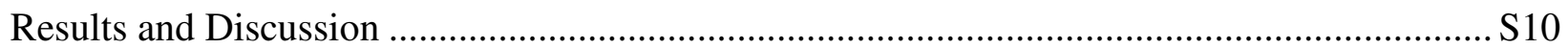

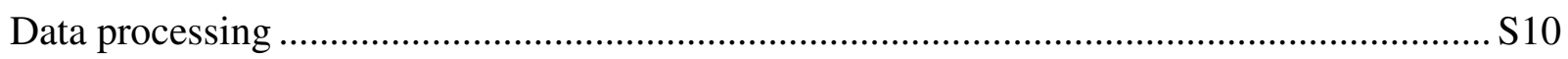

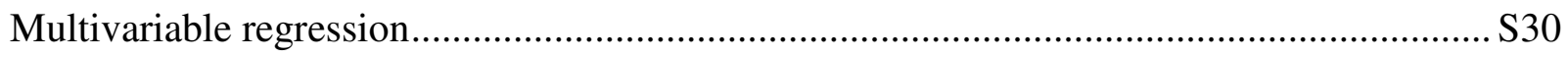

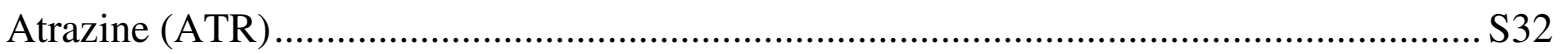

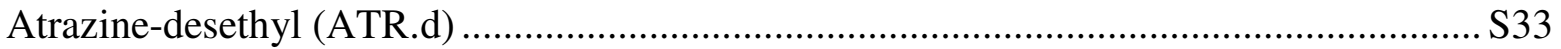

Atrazine-2-hydroxy (ATR.h) ....................................................................................... S34

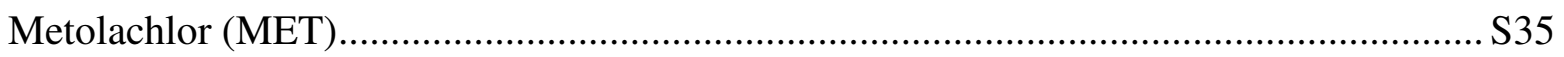

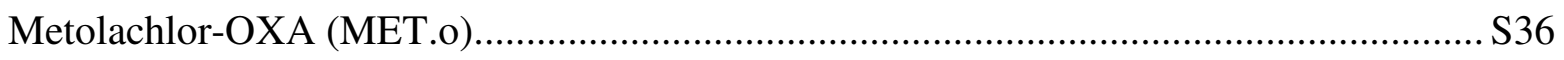

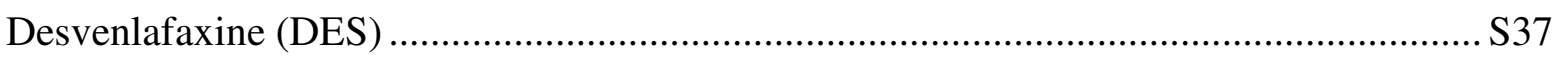

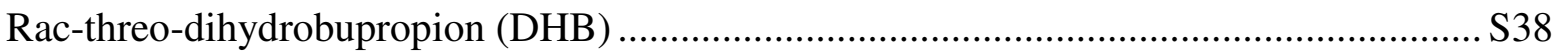

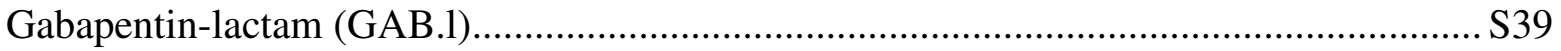

Cumulative normalized abundance (CUMA) ................................................................ S40

Cumulative estimated concentration (CUMC) …………................................................ S41

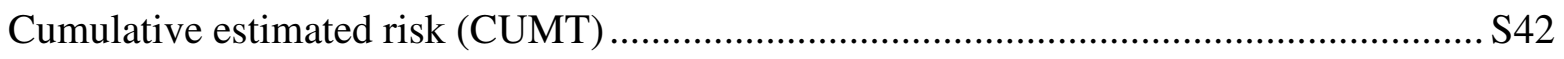

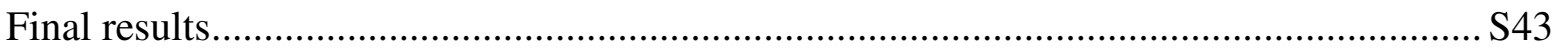

Recommendations for future micropollutant sampling strategies......................................... S46

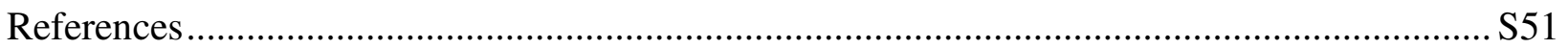




\section{List of Tables}

Table S1: Regression results using the final averaged coefficients for micropollutant dynamics and peak events of representative micropollutant profiles. Significant covariates are listed for each micropollutant profile with the sign of the relationship (+/-). Final model diagnostics $\left(\mathrm{R}^{2}\right.$ and area under curve, AUC) represent each imputed dataset (average \pm s.d.). S43

Table S2: Regression results using the final averaged coefficients for peak events of cumulative profiles. Significant covariates are listed for each micropollutant profile with the sign of the relationship (+/-). Final model diagnostics (area under curve, AUC) represent each imputed dataset (average \pm s.d.).

Table S3: Percent of environmental risk captured using uninformed and informed micropollutant sampling strategies. Informed strategies included the final peak event model and important bivariate models. The percent of environmental risk captured by each sampling strategy was determined by comparing the areas under the resulting profiles with the areas under the actual representation of risk profiles. S46

\section{List of Figures}

Figure S1: Daily stream flowrate $\left(\mathrm{m}^{3} \cdot \mathrm{s}^{-1}\right)$ duration curves for $2000-2018$ (black lines) and the study period (red line).

Figure S2: Elevations of the Fall Creek watershed (delineated by the black boundary) and surrounding area along with locations of the 92 NOAA weather stations (blue circles) and 6 NRCC weather stations (red triangles). S9

Figure S3: Missing values (red) throughout the study period. .......................................... S10

Figure S4: Temporal profile of desvenlafaxine (DES) concentration in $\mathrm{ng} \cdot \mathrm{L}^{-1} \ldots \ldots \ldots \ldots \ldots \ldots \ldots . \mathrm{S} 11$ Figure S5: Temporal profile of rac-threo-dihydrobupropion (DHB) concentration in $\mathrm{ng} \cdot \mathrm{L}^{-1}$. . S11 Figure S6: Temporal profile of gabapentin-lactam (GAB.1) concentration in $\mathrm{ng} \cdot \mathrm{L}^{-1}$............. S12

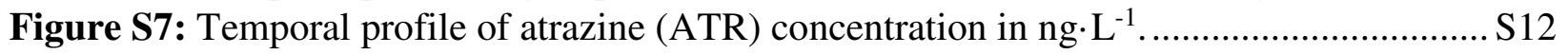
Figure S8: Temporal profile of atrazine-desethyl (ATR.d) concentration in $\mathrm{ng} \cdot \mathrm{L}^{-1}$............... S13 Figure S9: Temporal profile of atrazine-2-hydroxy (ATR.h) concentration in $\mathrm{ng} \cdot \mathrm{L}^{-1} \ldots \ldots \ldots \ldots . \mathrm{S} 13$ Figure S10: Temporal profile of metolachlor (MET) concentration in $\mathrm{ng} \cdot \mathrm{L}^{-1} \ldots \ldots \ldots \ldots \ldots \ldots \ldots . \mathrm{S} 14$ Figure S11: Temporal profile of metolachlor-OXA (MET.o) concentration in $n g \cdot \mathrm{L}^{-1}$........... S14 Figure S12: Temporal profile of cumulative normalized intensity (CUMA), which represents the sum of 90 previously reported micropollutants (i.e., temporal abundance profiles of all 90 detected micropollutants were normalized to the maximum abundance in each profile and then summed together). S15

Figure S13: Temporal profile of cumulative estimated concentration (CUMC) in $n g \cdot \mathrm{L}^{-1}$, which represents 42 micropollutants that we previously identified with level 1 confidence. S15 Figure S14: Temporal profile of cumulative estimated concentration (CUMC2) in $\mathrm{ng} \cdot \mathrm{L}^{-1}$, which represents the 32 micropollutants that were consistently measured at levels below $1 \mu \mathrm{g} \cdot \mathrm{L}^{-1}$ over the duration of the study period. S16 
Figure S15: Temporal profile of cumulative estimated risk (CUMT) as exposure-activity ratio (EAR), which represents the 17 micropollutants that were identified with level 1 confidence and for which toxicity data was available............................................................................. S16

Figure S16: Temporal profile of total daily precipitation ( $\mathrm{prcp}$ ) in $\mathrm{mm}$. ............................. S17 Figure S17: Temporal profile of daily precipitation index ( rcp01 - binary value describing days

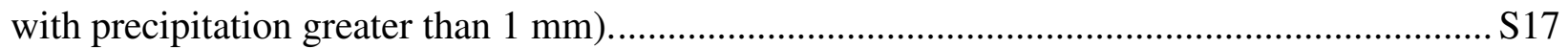

Figure S18: Temporal profile of daily antecedent dry period $(a d p)$ in days. ......................... S18

Figure S19: Temporal profile of average daily air temperature $(\operatorname{tavg})$ in ${ }^{\circ} \mathrm{C}$. ......................... S18

Figure S20: Temporal profile of average maximum daily air temperature $(\operatorname{tmax})$ in ${ }^{\circ} \mathrm{C}$......... S19

Figure S21: Temporal profile of average minimum daily air temperature $(t m i n)$ in ${ }^{\circ} \mathrm{C}$............ S19

Figure S22: Temporal profile of average daily snowfall (snow) in mm............................. S20

Figure S23: Temporal profile of average daily snow depth $(s n w d)$ in $\mathrm{mm}$............................ S20

Figure S24: Temporal profile of average daily snow melt (snmt) in mm............................ S21

Figure S25: Temporal profile of average daily wind speed (awnd) in $\mathrm{m} \cdot \mathrm{s}^{-1} \ldots \ldots \ldots \ldots \ldots \ldots \ldots \ldots . \mathrm{S} 21$

Figure S26: Temporal profile of total daily leaf wetness (lwet) in min............................... S22

Figure S27: Temporal profile of total daily solar radiation ( $\mathrm{srad}$ ) in langleys....................... S22

Figure S28: Temporal profile of average daily relative humidity (rhum) in \% ......................S23

Figure S29: Temporal profile of average daily streamflow rate (flow; solid line) and baseflow rate

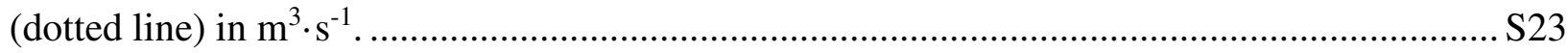

Figure S30: Temporal profile of average daily baseflow index $(b f i)$................................. S24 Figure S31: Temporal profile of average daily sewage treatment plant discharge $(s t p)$ in $\mathrm{m}^{3} \cdot \mathrm{s}^{-1}$.

Figure S32: Temporal profile of average daily sewage proportion ( $p s t p)$ in \% ................... S25

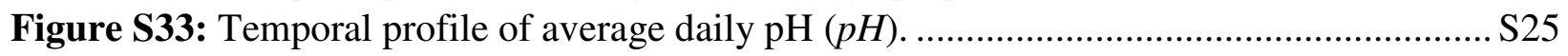

Figure S34: Temporal profile of average daily turbidity $(t u r b)$ in NTU.............................. S26

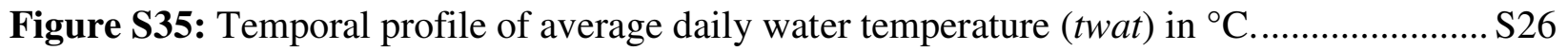

Figure S36: Temporal profile of average daily $\mathrm{UV}_{254}$ absorbance $(u v)$.............................. S27

Figure S37: Temporal profile of daily alkalinity measurements (alk) in $\mathrm{mg} \cdot \mathrm{L}^{-1}$ as $\mathrm{CaCO}_{3} \ldots . . . \mathrm{S} 27$

Figure S38: Temporal profile of average daily water sample age (age) in days....................S28

Figure S39: Optimal growing seasons for atrazine as determined by correlation with bfi. Optimal start dates are marked with a dashed line.

Figure S40: Environmental covariates (average of the imputed datasets) correlation matrix.

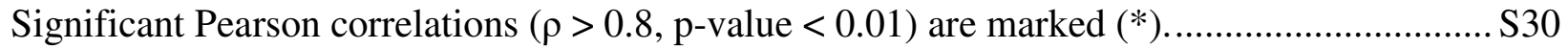
Figure S41: Cross-validation time series; blue lines represent training sets and red lines represent

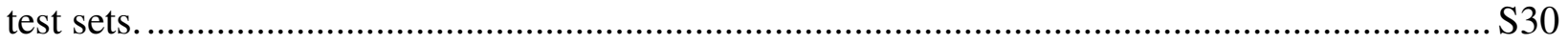

Figure S42: Bivariate GLS regression plots for ATR (left) and significant final standardized coefficients (right).

Figure S43: Bivariate logit regression plots for ATR (left) and significant final standardized coefficients (right) during the growing season.

Figure S44: Bivariate GLS regression plots for ATR.d (left) and significant final standardized coefficients (right).

Figure S45: Bivariate logit regression plots for ATR.d (left) and significant final standardized coefficients (right) during the growing season. S33 
Figure S46: Bivariate GLS regression plots for ATR.h (left) and significant final standardized coefficients (right).

Figure S47: Bivariate logit regression plots for ATR.h (left) and significant final standardized coefficients (right).

Figure S48: Bivariate GLS regression plots for MET (left) and significant final standardized coefficients (right).

Figure S49: Bivariate logit regression plots for MET (left) and significant final standardized coefficients (right) during the growing season.

Figure S50: Bivariate GLS regression plots for MET.o (left) and significant final standardized coefficients (right).

Figure S51: Bivariate logit regression plots for MET.o (left) and significant final standardized coefficients (right).

Figure S52: Bivariate GLS regression plots for DES (left) and significant final standardized coefficients (right).

Figure S53: Bivariate logit regression plots for DES (left) and significant final standardized coefficients (right).

Figure S54: Bivariate GLS regression plots for DHB (left) and significant final standardized coefficients (right).

Figure S55: Bivariate logit regression plots for DHB (left) and significant final standardized coefficients (right).

Figure S56: Bivariate GLS regression plots for GAB.1 (left) and significant final standardized coefficients (right).

Figure S57: Bivariate logit regression plots for GAB.l (left) and significant final standardized coefficients (right).

Figure S58: Bivariate logit regression plots for CUMA (left) and significant final standardized coefficients (right).

Figure S59: Bivariate logit regression plots for CUMC (left) and significant final standardized coefficients (right).

Figure S60: Bivariate logit regression plots for CUMC2 (left) and significant final standardized coefficients (right).

Figure S61: Bivariate logit regression plots for CUMT (left) and significant final standardized coefficients (right).

Figure S62: Informed vs uninformed sampling strategies for desvenlafaxine (DES) concentration in $\mathrm{ng} \cdot \mathrm{L}^{-1}$; solid black line $=$ average of the imputed datasets; green points = triggered sampling events $(\geq 2)$; dashed black line $=$ actual representation of risk; blue area $=$ informed sampling, red area $=$ uninformed sampling.

Figure S63: Informed vs uninformed sampling strategies for cumulative estimated concentration $(\mathrm{CUMC})$ in $\mathrm{ng} \cdot \mathrm{L}^{-1}$; solid black line = average of the imputed datasets; green points = triggered sampling events $(\geq 2)$; dashed black line = actual representation of risk; blue area = informed sampling, red area $=$ uninformed sampling..... S48

Figure S64: Informed vs uninformed sampling strategies for cumulative estimated concentration $\left(\right.$ CUMC2) in ng. $\mathrm{L}^{-1}$; solid black line = average of the imputed datasets; green points = triggered 
sampling events $(\geq 2)$; dashed black line = actual representation of risk; blue area $=$ informed sampling, red area $=$ uninformed sampling

Figure S65: Informed vs uninformed sampling strategies for cumulative estimated risk (CUMT) expressed as exposure-activity ratio (EAR); solid black line = average of the imputed datasets; green points $=$ triggered sampling events $(\geq 2)$; dashed black line $=$ actual representation of risk; blue area $=$ informed sampling, red area $=$ uninformed sampling. S50 


\section{Materials and methods}

Data collection - micropollutant profiles.

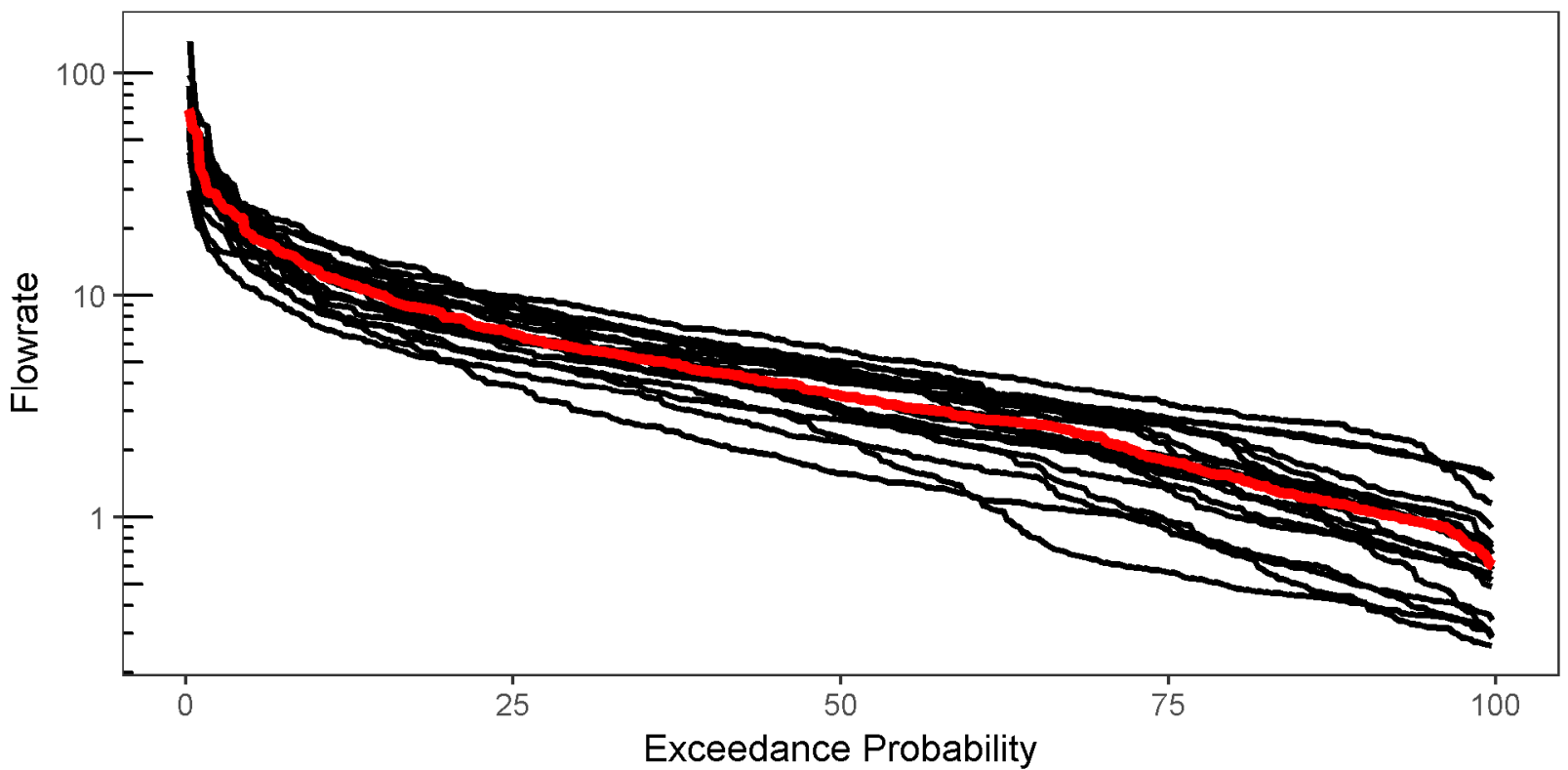

Figure S1: Daily stream flowrate $\left(\mathrm{m}^{3} \cdot \mathrm{s}^{-1}\right)$ duration curves for $2000-2018$ (black lines) and the study period (red line). 
Data collection - environmental covariates. Daily weather data were obtained from the National Oceanic and Atmospheric Administration (NOAA) ${ }^{1}$ using the R package $r n o a a^{2}$ and from the Northeast Regional Climate Center (NRCC). ${ }^{3}$ A list of 92 NOAA and 6 NRCC weather stations were identified within a distance of approximately $66 \mathrm{~km}$ (4x the watershed radius) from the watershed centroid. A map of the weather stations is provided in Figure S2. Spatial interpolations for daily precipitation ( $\operatorname{srcp}$ ) data were conducted by first filling missing data from individual weather stations using inverse distance weighting and then applying temporally pooled kriging using the $\mathrm{R}$ package sstat $^{4}{ }^{4}$ The precipitation index (prcp01) is a binary factor to reflect days with precipitation; prcp01 specifically represents days with greater than $1 \mathrm{~mm}$ of precipitation as a value of 1 , and 0 otherwise. The antecedent dry period $(a d p)$ for each day was determined by the number of previous days since an average rainfall event greater than $1 \mathrm{~mm}$. All other weather data (minimum temperature, tmin; average temperature, tavg; maximum temperature, tmax; snowfall, snow; snow depth, snwd; wind speed, awnd; leaf wetness, lwet; solar radiation, srad; and relative humidity, rhum) were averaged across the watershed from all available weather stations for each day using Thiessen polygons and the R package spatstat. ${ }^{5}$ Daily snow melt (snmt) was determined by the difference between snwd on the preceding day. Streamflow rates (flow) were obtained from a U.S. Geological Survey (USGS) stream gage located less than $2 \mathrm{~km}$ downstream of the Fall Creek Monitoring Station (FCMS). ${ }^{6}$ Baseflow rates were calculated using the R package $l f s t a t^{7}$ to determine the average baseflow index (bfi) for each day. Average daily sewage treatment plant (STP) discharge rates (stp) were obtained from one of the two STPs that discharge directly into Fall Creek and normalized by flow to determine the contribution of stp to flow, or the sewage proportion ( $p s t p)$. Data from the other STP could not be obtained. Water quality parameters that are regularly collected at the Cornell Water Filtration Plant through continuous in-line 
measurements ( $p H$; turbidity, turb; and water temperature, twat) and daily grab samples ( $\mathrm{UV}_{254}$ absorbance, $u v$; and alkalinity, alk) of the raw water were also included as environmental covariates. Lastly, the age of the water sample (age) determined as the number of days between sample collection and preparation, was also included to account for potential micropollutant degradation during storage.

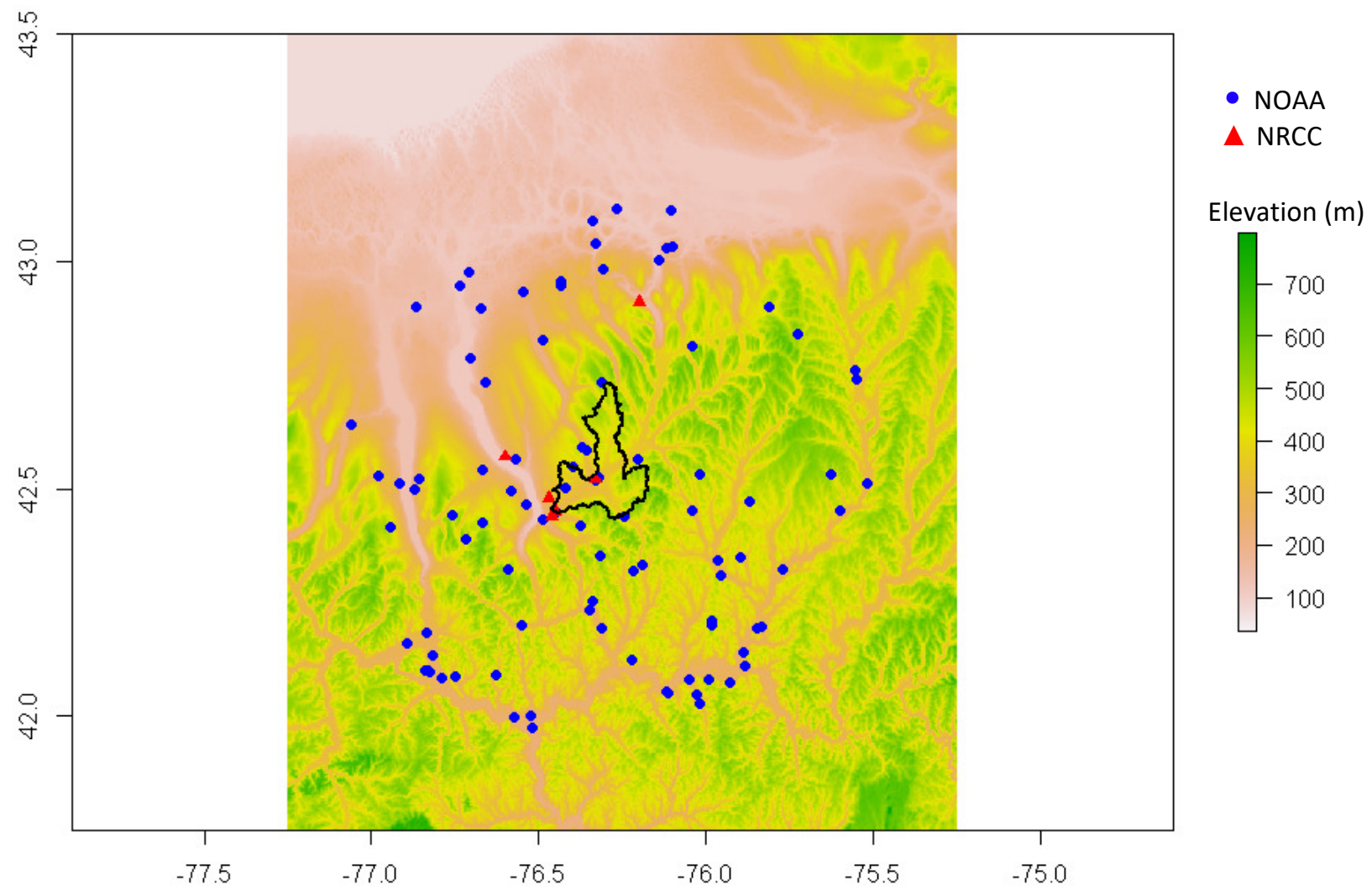

Figure S2: Elevations of the Fall Creek watershed (delineated by the black boundary) and surrounding area along with locations of the 92 NOAA weather stations (blue circles) and 6 NRCC weather stations (red triangles). 


\section{Results and Discussion}

\section{Data processing}

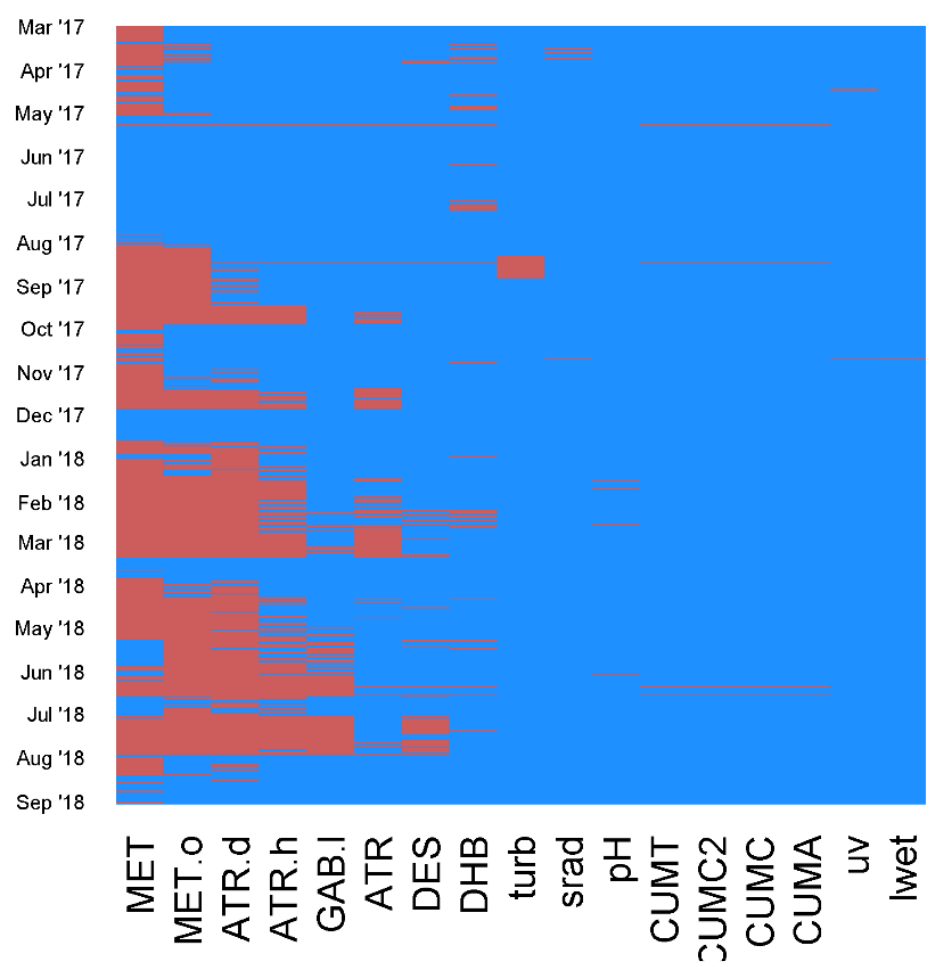

Figure S3: Missing values (red) throughout the study period.

$(\mathrm{MET}=63.2 \%$ missing; MET.o $=47.5 \%$; ATR.d $=43.3 \% ;$ ATR.h $=28.4 \% ;$ GAB.l $=12.1 \%$; ATR $=11.8 \% ; \mathrm{DES}=8.0 \% ; \mathrm{DHB}=6.2 \% ;$ turb $=2.9 \% ; \mathrm{srad}=0.7 \% ; \mathrm{pH}=0.7 \% ; \mathrm{CUMC}=0.7 \%$; $\mathrm{CUMA}=0.7 \% ; \mathrm{CUMT}=0.7 \%$; $\mathrm{uv}=0.4 \%$; and lwet $=0.2 \%$ ).

Complete temporal profiles for the representative individual micropollutant concentrations (Figures S4 - S11), cumulative metrics of overall micropollutant contamination (Figures S12 S15), and environmental covariates (Figures S16 - S38 are provided below. The black line represents the measured data. Red segments represent the standard deviation of the five imputed results. For the temporal profiles of micropollutant concentrations and cumulative metrics of overall micropollutant contamination, the blue line represents the peak event limit (highest quartile of values) and the green shaded areas represent the apparent growing seasons. 


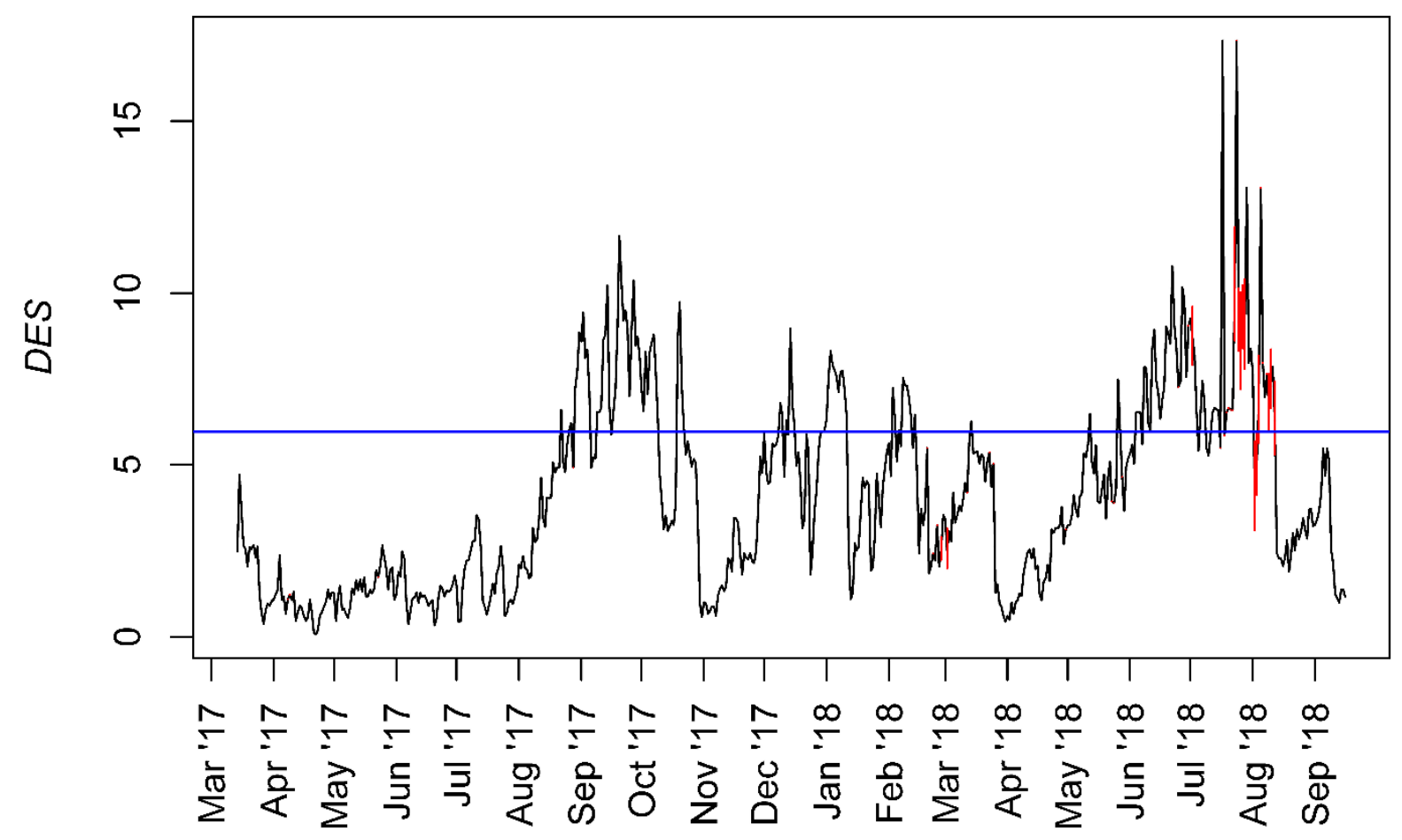

Figure S4: Temporal profile of desvenlafaxine (DES) concentration in $n g \cdot \mathrm{L}^{-1}$.

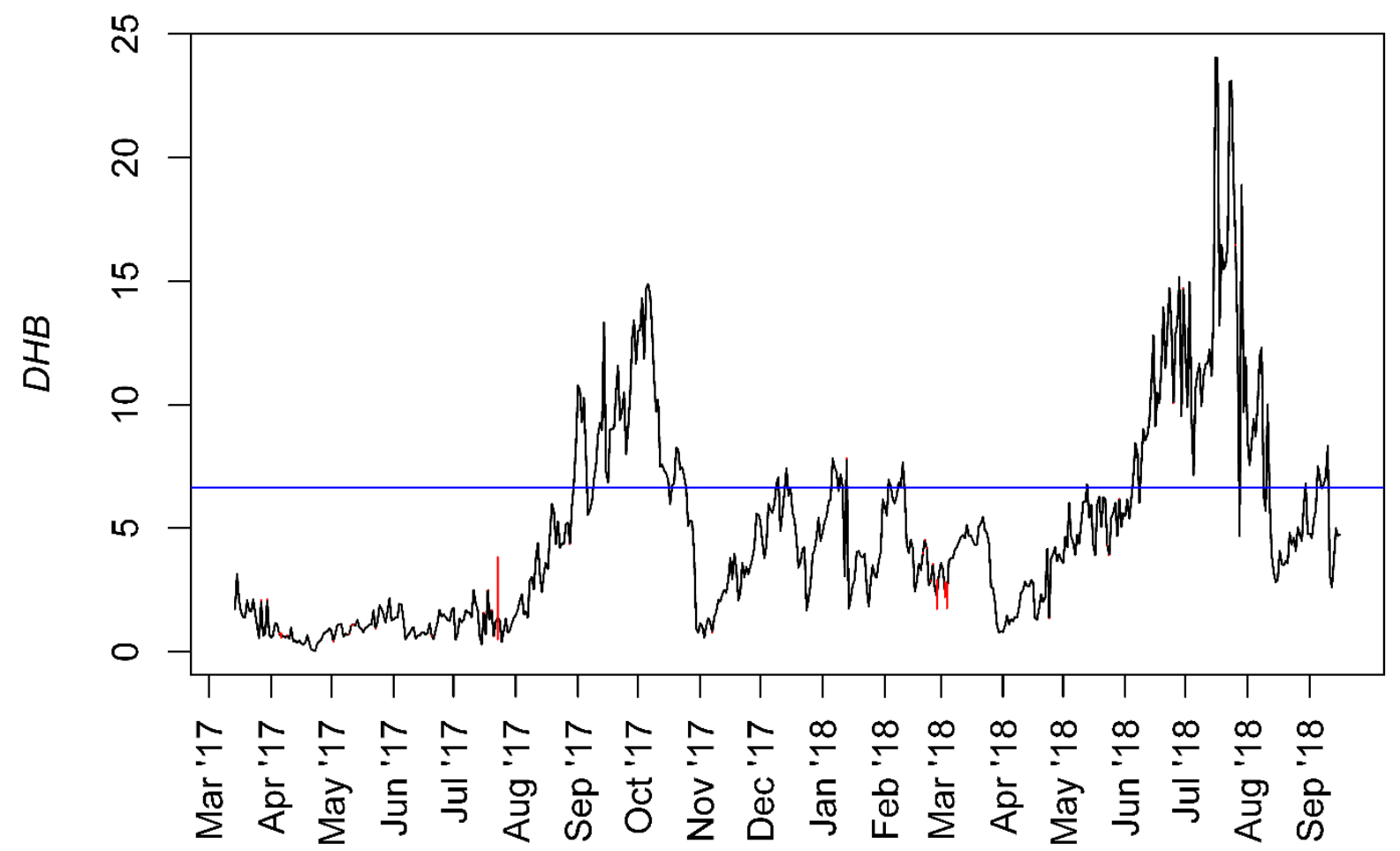

Figure S5: Temporal profile of rac-threo-dihydrobupropion (DHB) concentration in $\mathrm{ng} \cdot \mathrm{L}^{-1}$. 


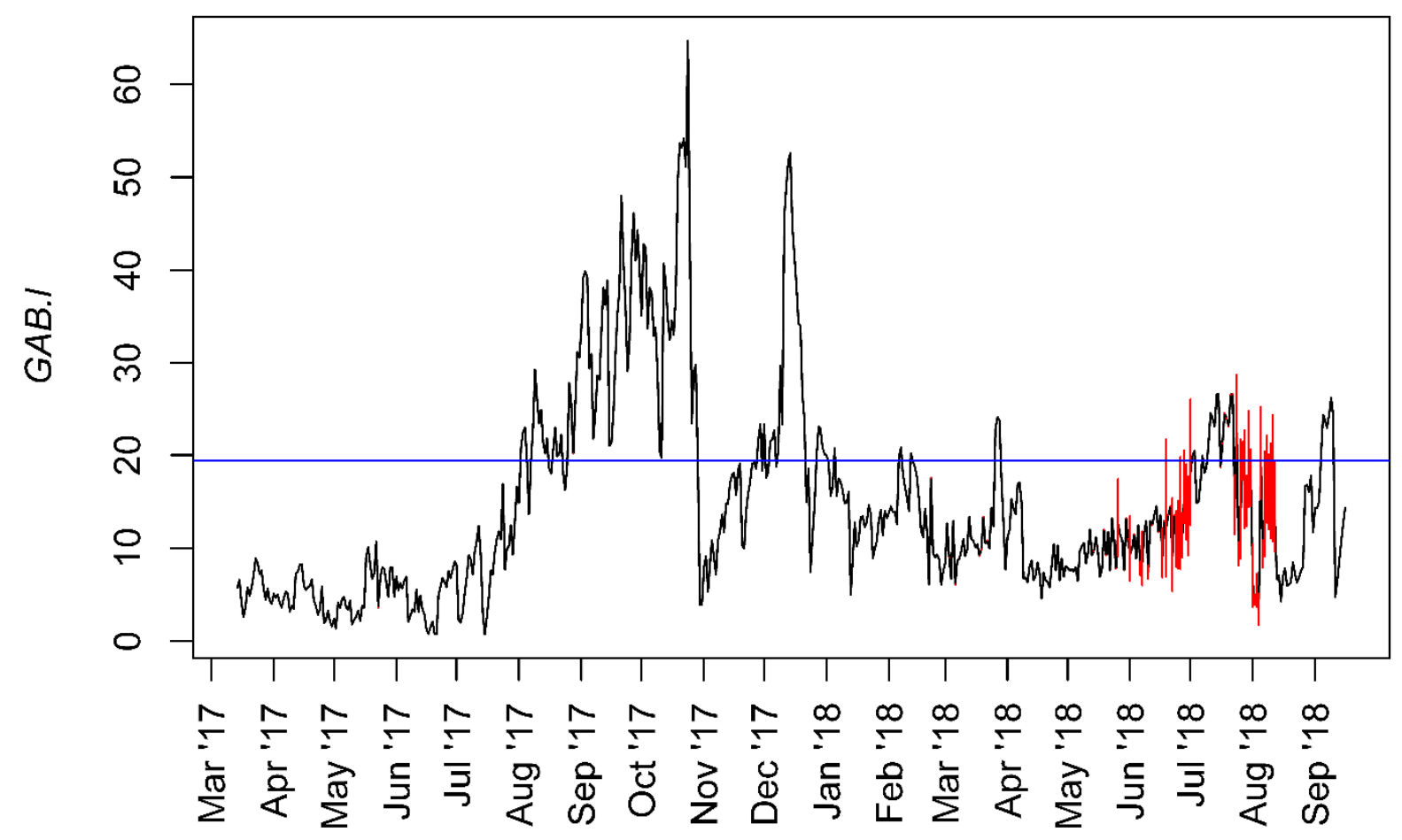

Figure S6: Temporal profile of gabapentin-lactam (GAB.l) concentration in $\mathrm{ng} \cdot \mathrm{L}^{-1}$.

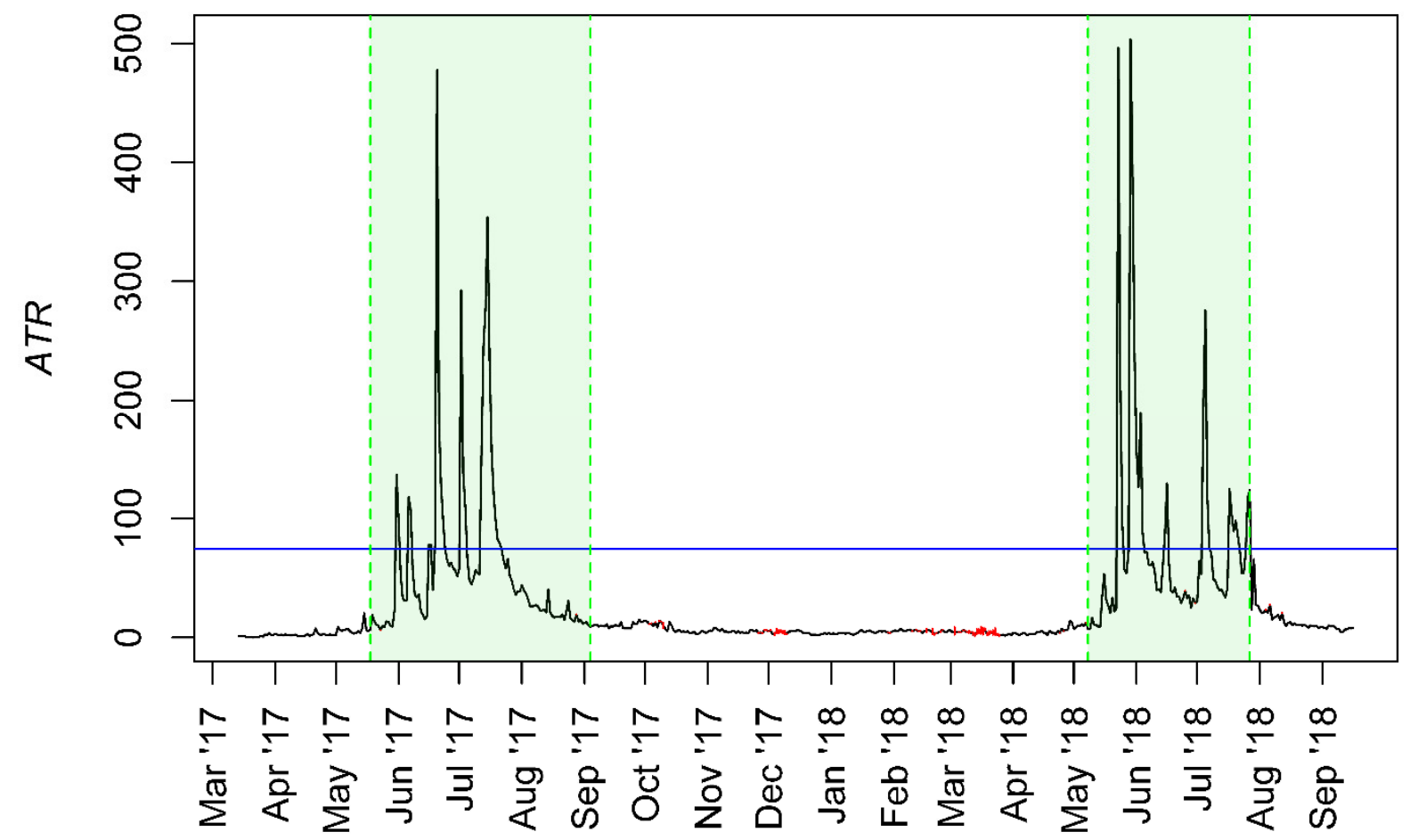

Figure S7: Temporal profile of atrazine (ATR) concentration in $\mathrm{ng} \cdot \mathrm{L}^{-1}$. 


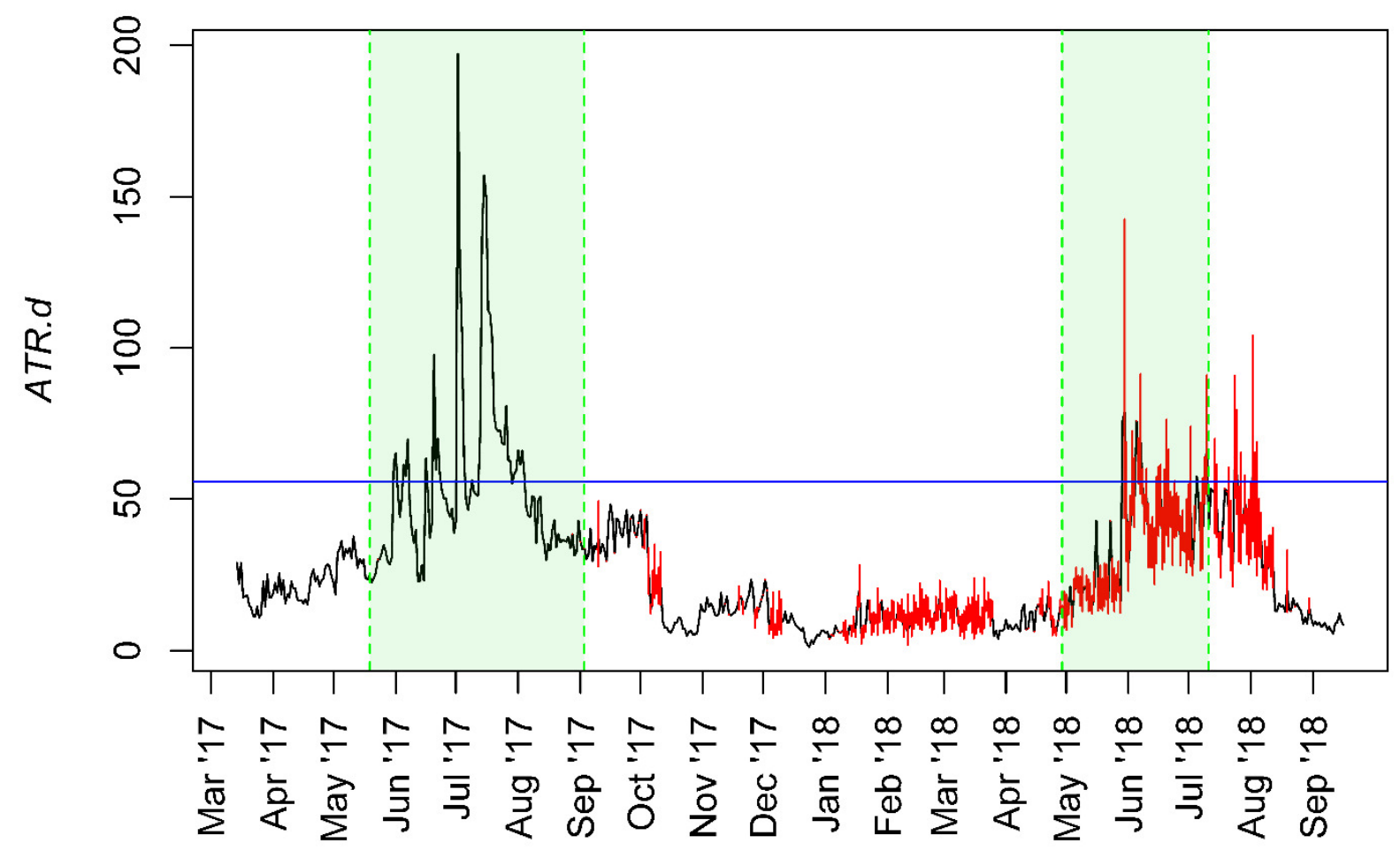

Figure S8: Temporal profile of atrazine-desethyl (ATR.d) concentration in $n g \cdot \mathrm{L}^{-1}$.

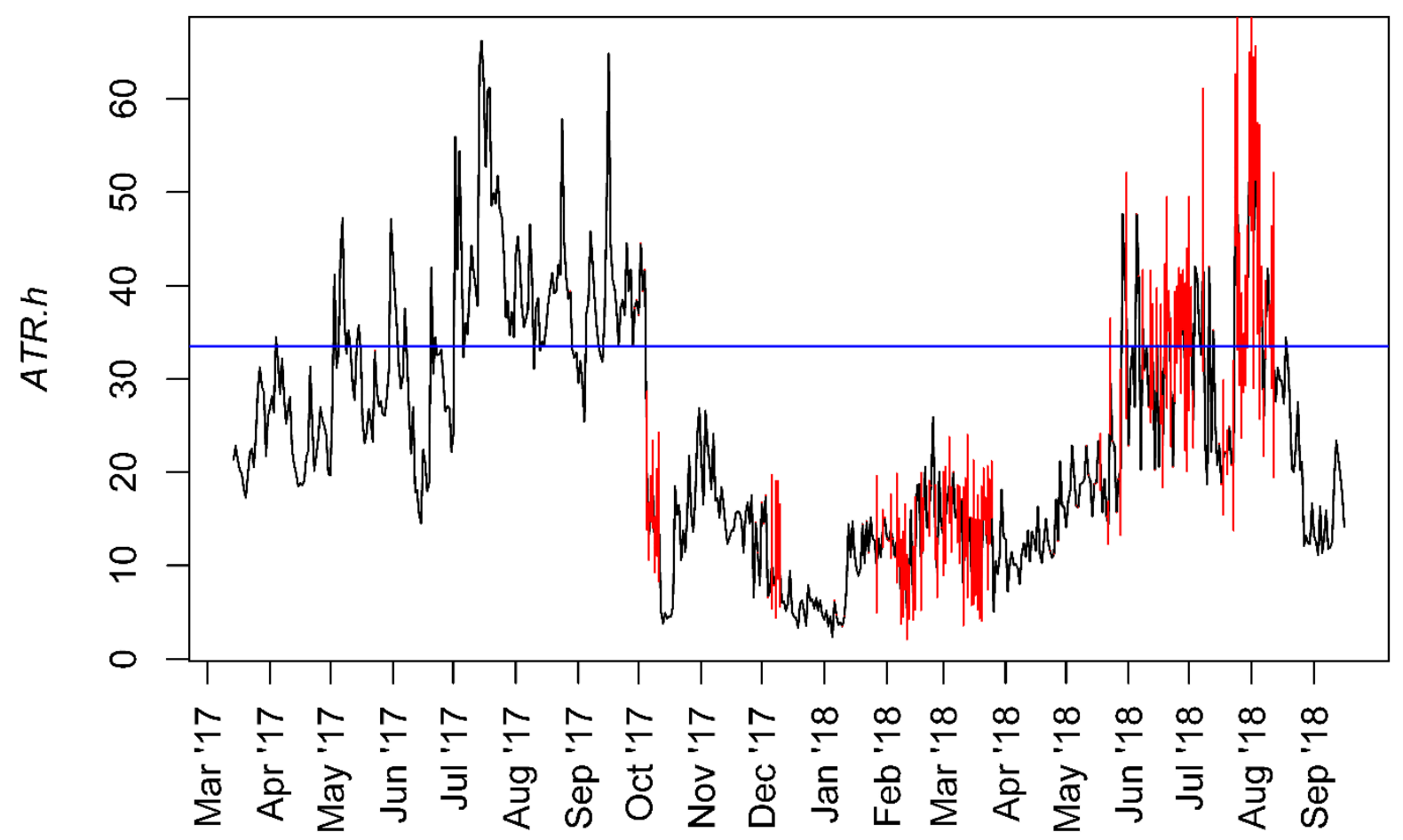

Figure S9: Temporal profile of atrazine-2-hydroxy (ATR.h) concentration in ng. $\mathrm{L}^{-1}$. 


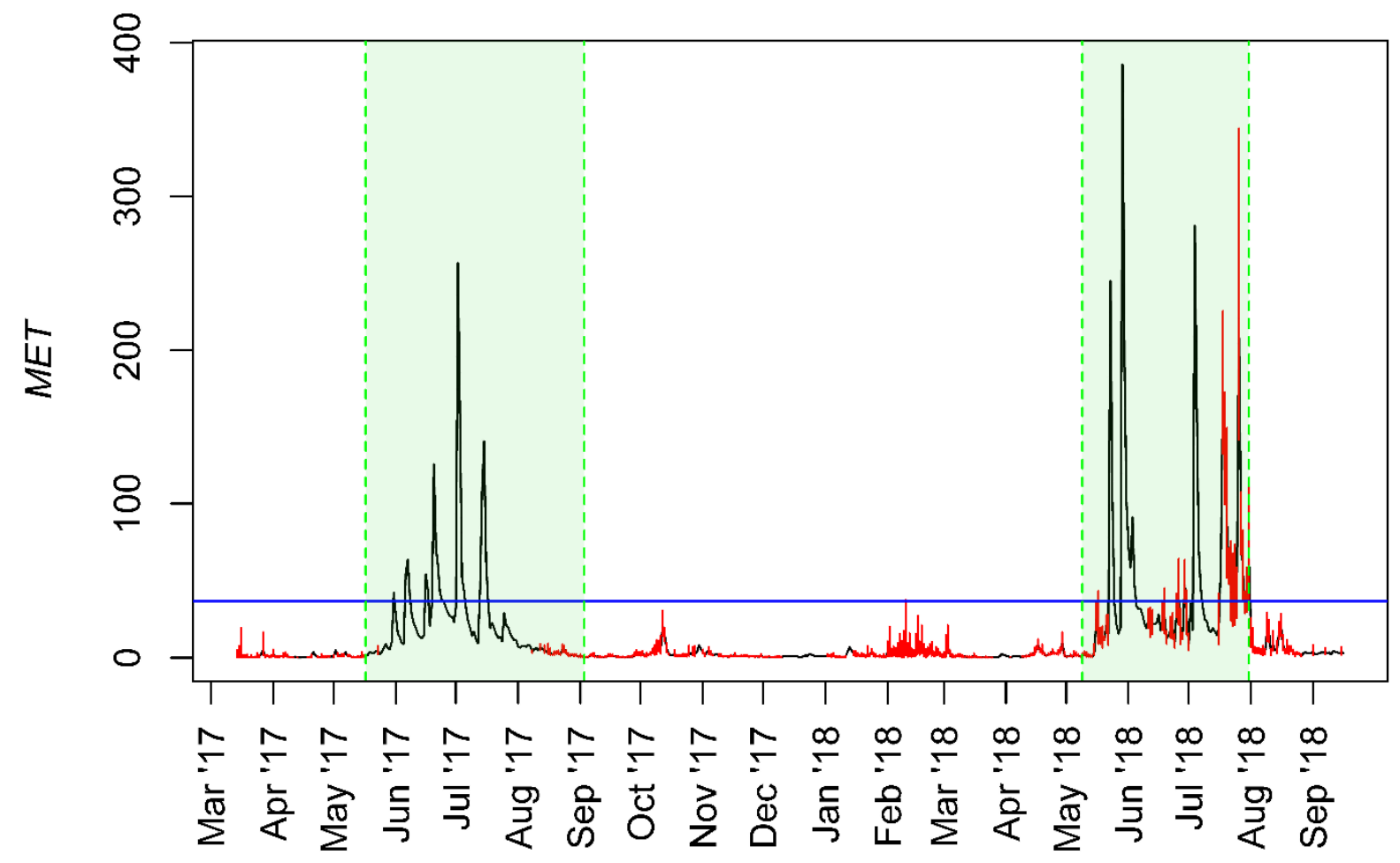

Figure S10: Temporal profile of metolachlor (MET) concentration in $n g \cdot \mathrm{L}^{-1}$.

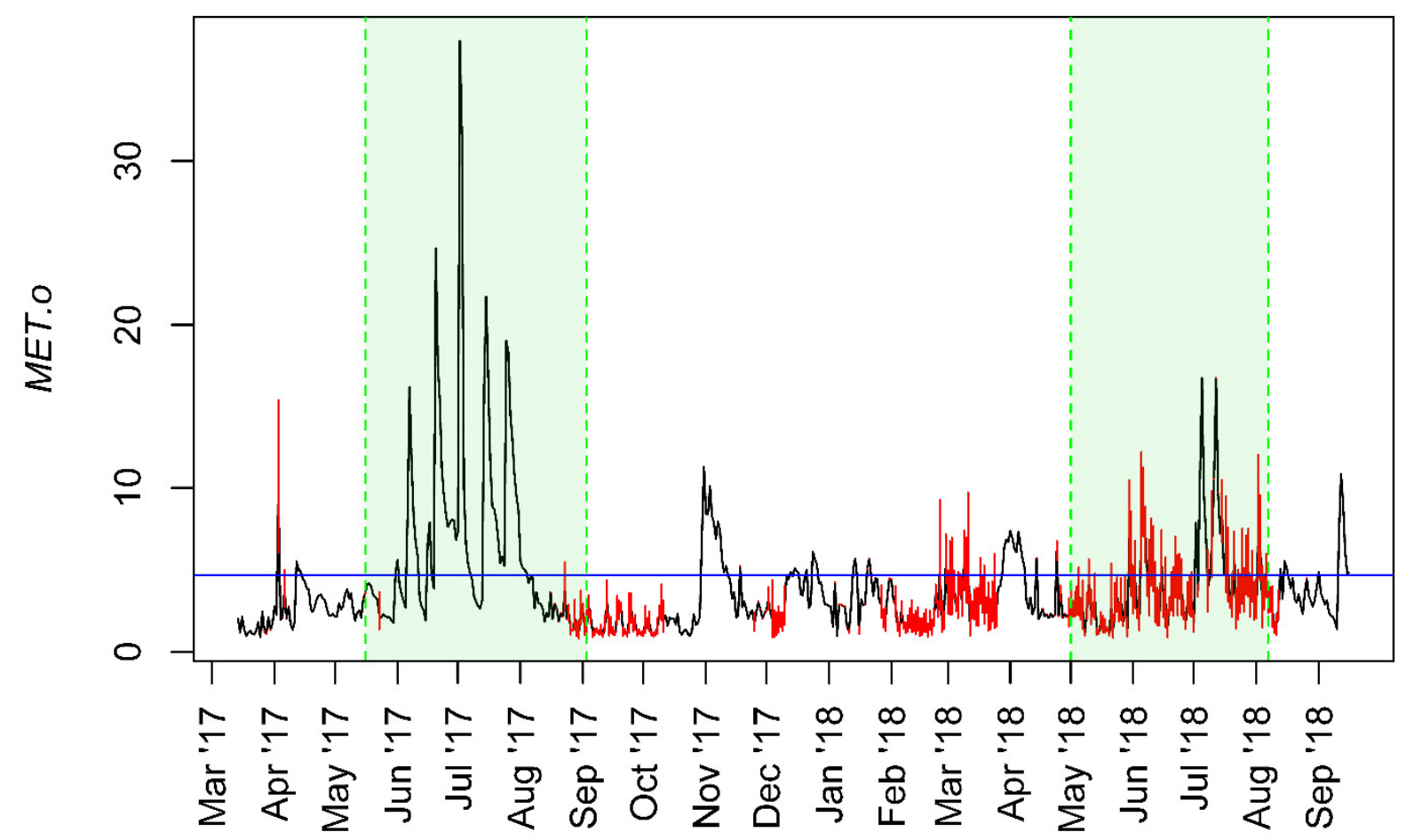

Figure S11: Temporal profile of metolachlor-OXA (MET.o) concentration in ng. $\mathrm{L}^{-1}$. 


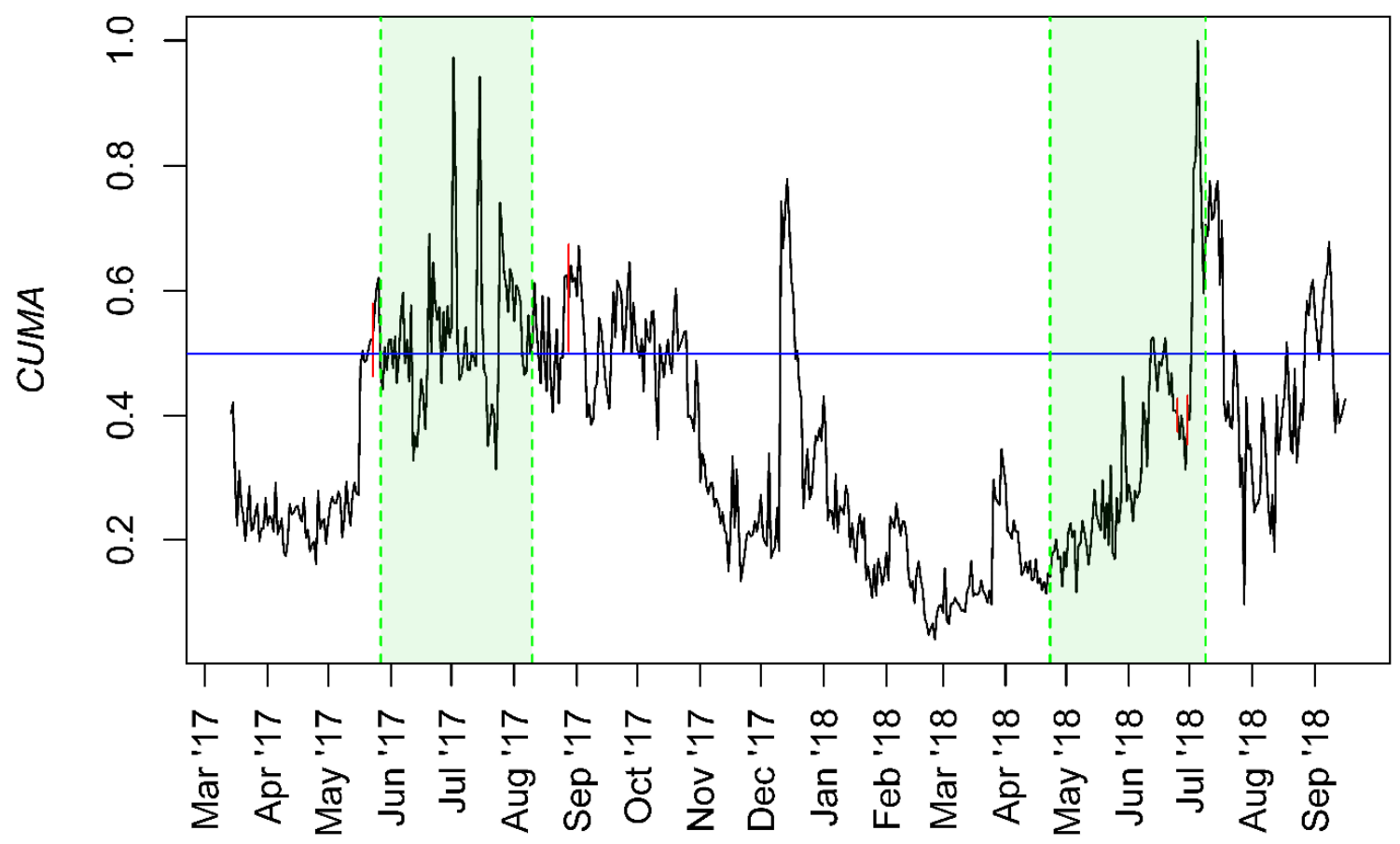

Figure S12: Temporal profile of cumulative normalized intensity (CUMA), which represents the sum of 90 previously reported micropollutants (i.e., temporal abundance profiles of all 90 detected micropollutants were normalized to the maximum abundance in each profile and then summed together).

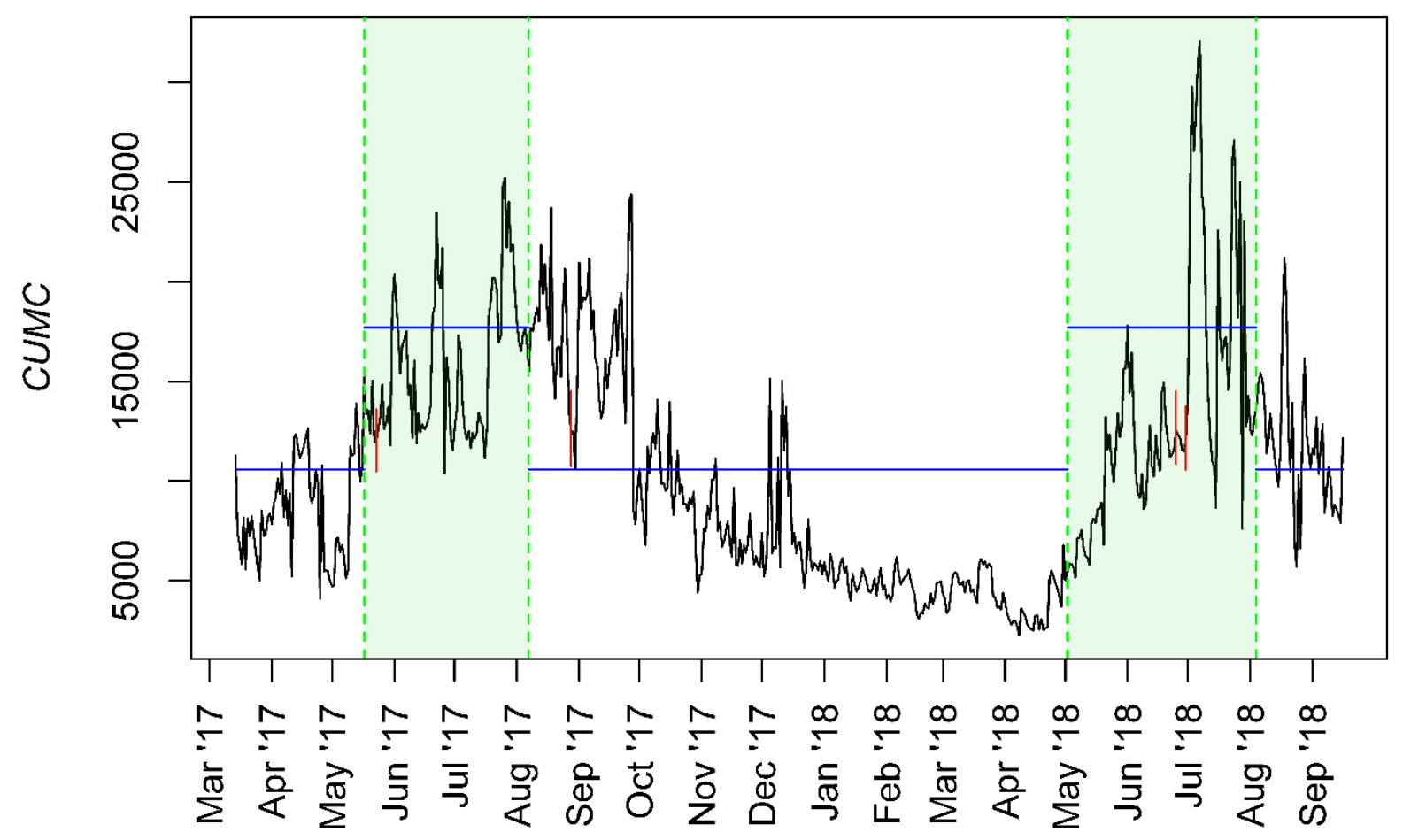

Figure S13: Temporal profile of cumulative estimated concentration (CUMC) in $\mathrm{ng} \cdot \mathrm{L}^{-1}$, which represents 42 micropollutants that we previously identified with level 1 confidence. 


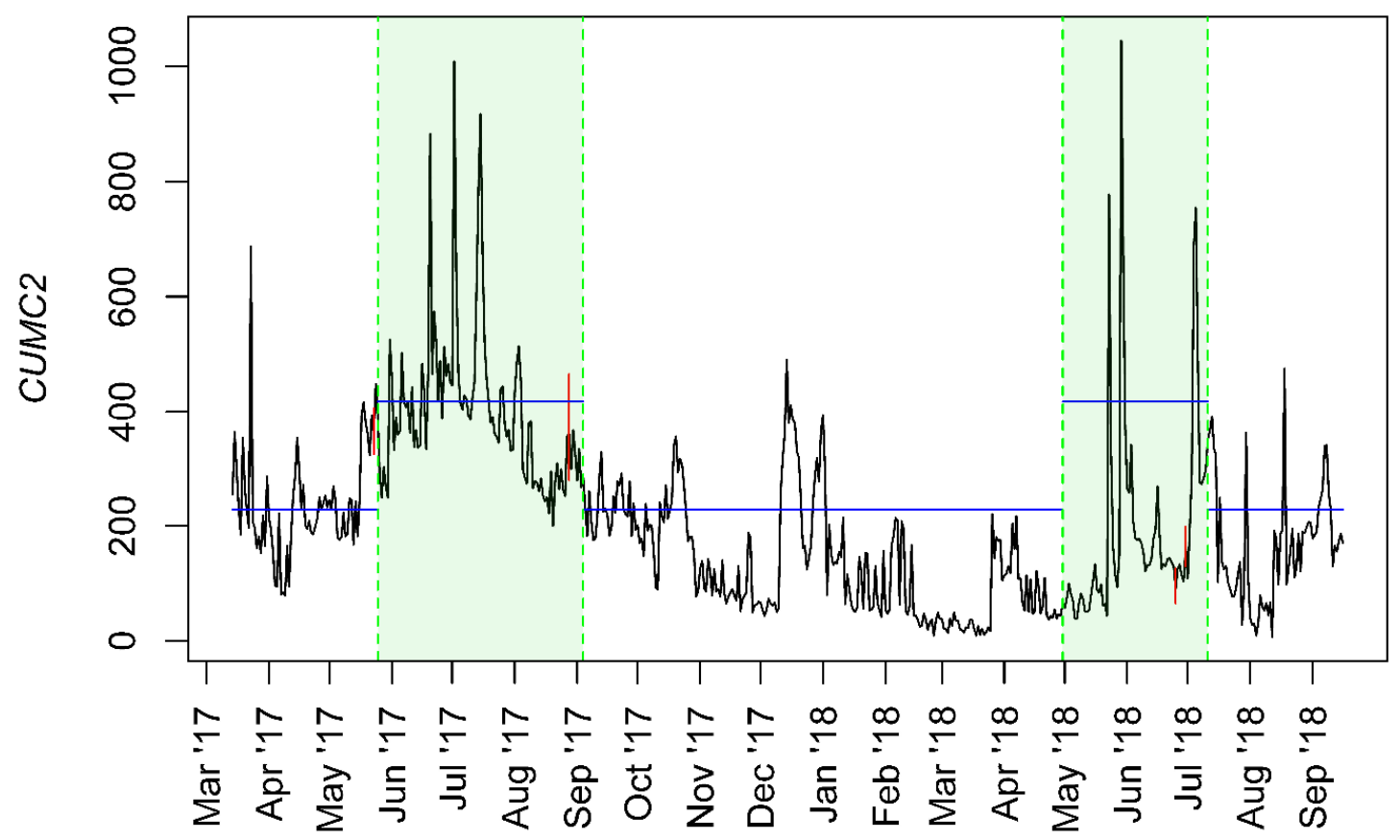

Figure S14: Temporal profile of cumulative estimated concentration (CUMC2) in $\mathrm{ng} \cdot \mathrm{L}^{-1}$, which represents the 32 micropollutants that were consistently measured at levels below $1 \mu \mathrm{g} \cdot \mathrm{L}^{-1}$ over the duration of the study period.

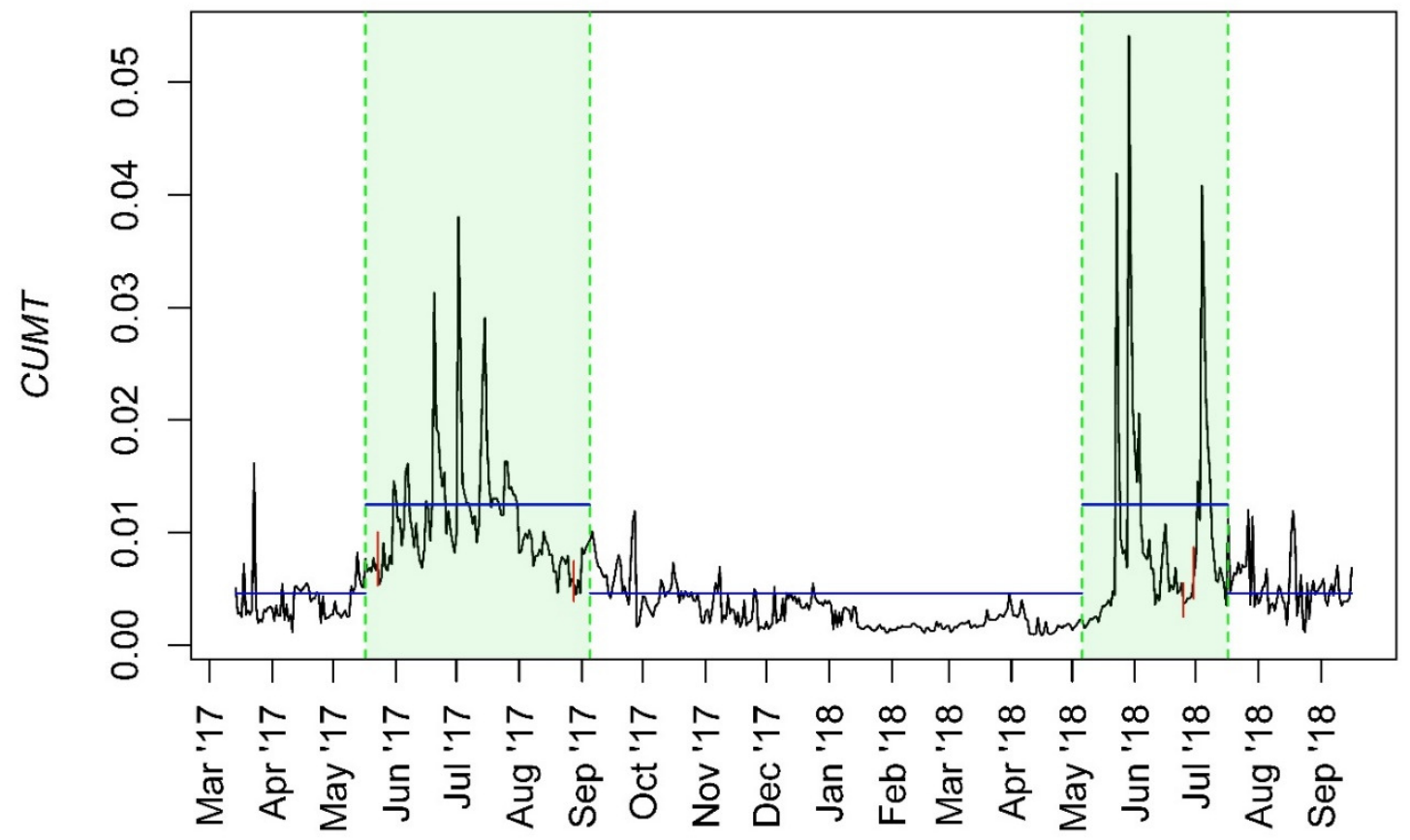

Figure S15: Temporal profile of cumulative estimated risk (CUMT) as exposure-activity ratio (EAR), which represents the 17 micropollutants that were identified with level 1 confidence and for which toxicity data was available. 


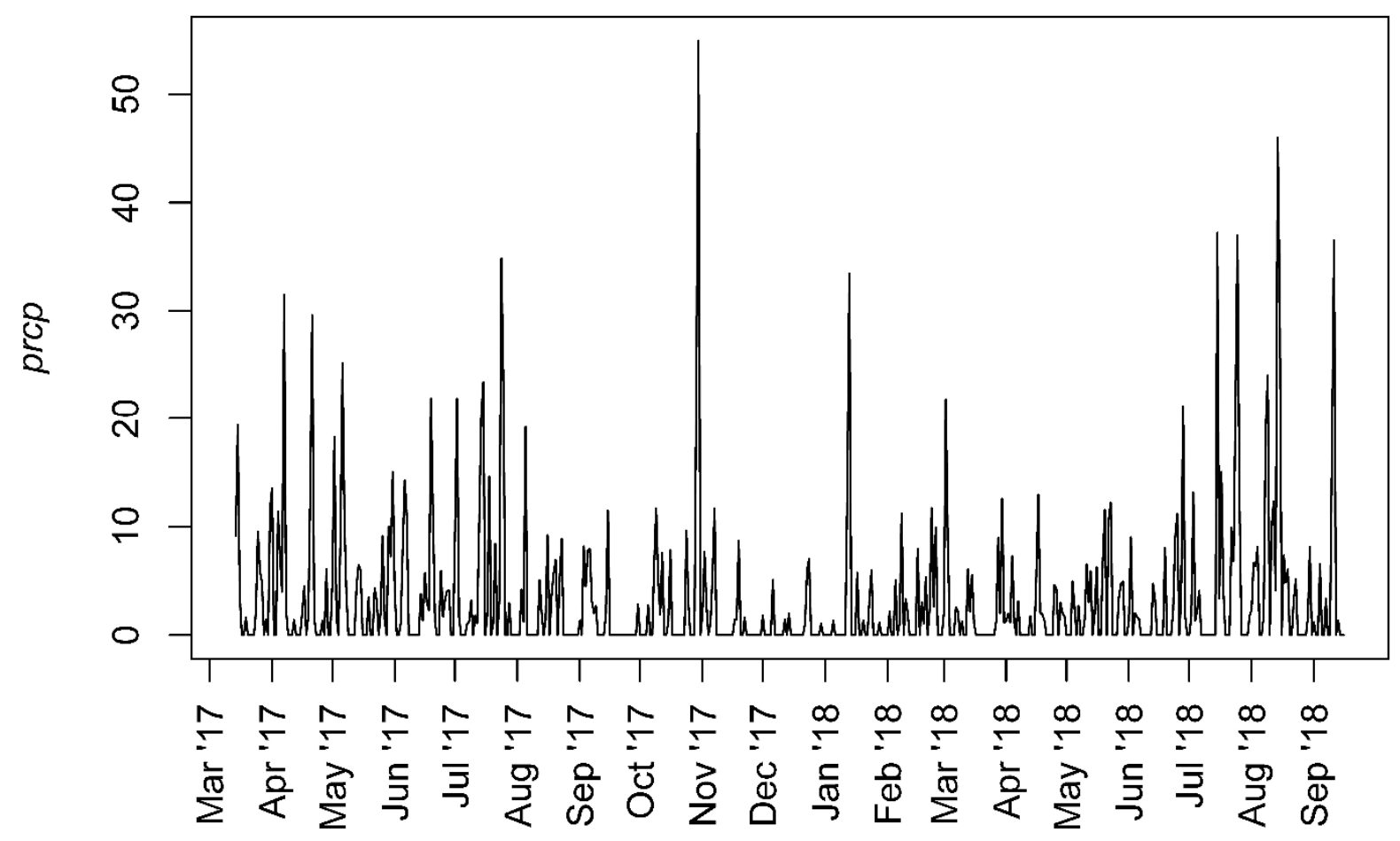

Figure S16: Temporal profile of total daily precipitation ( $p r c p)$ in $\mathrm{mm}$.

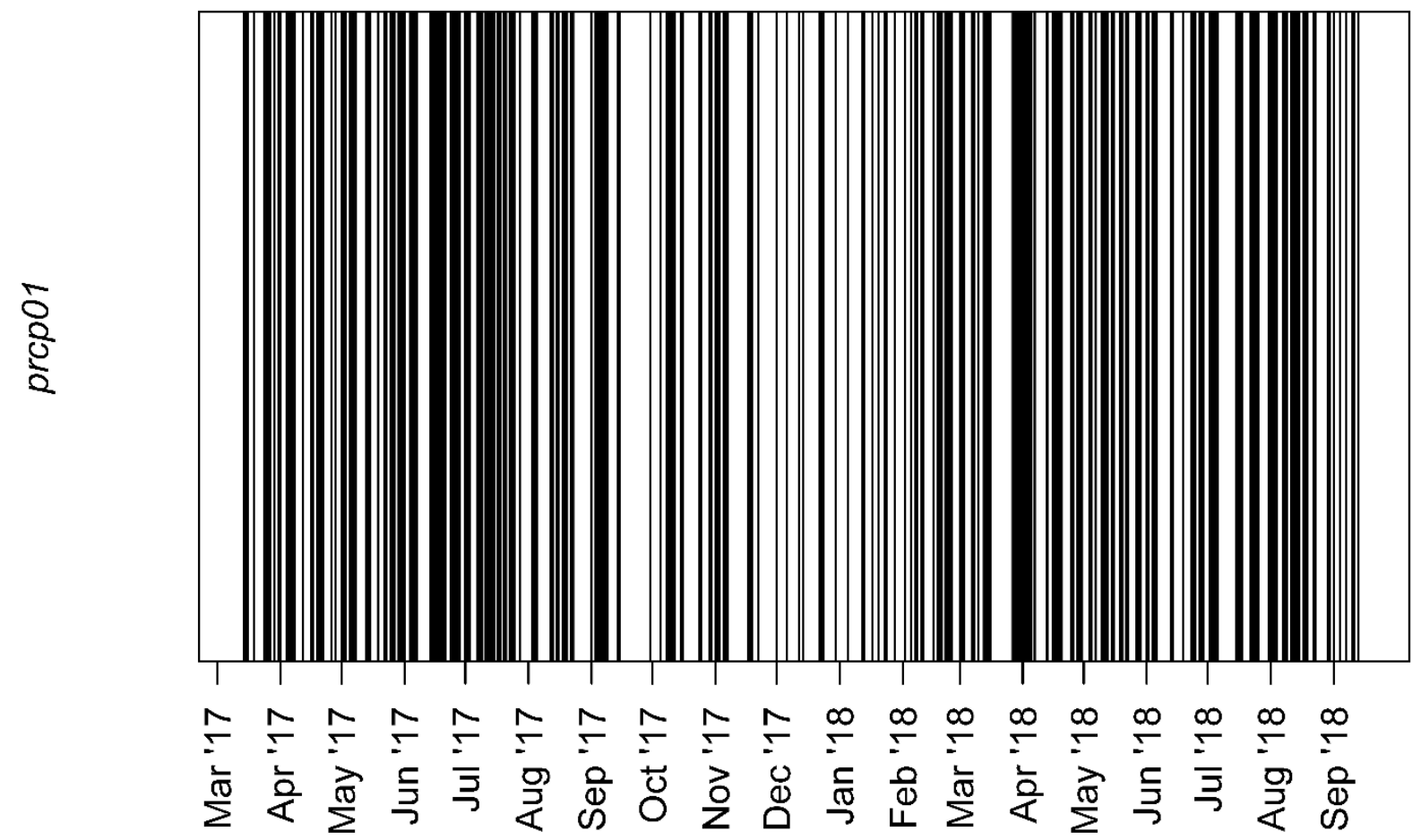

Figure S17: Temporal profile of daily precipitation index (prcp01 - binary value describing days with precipitation greater than $1 \mathrm{~mm}$ ). 


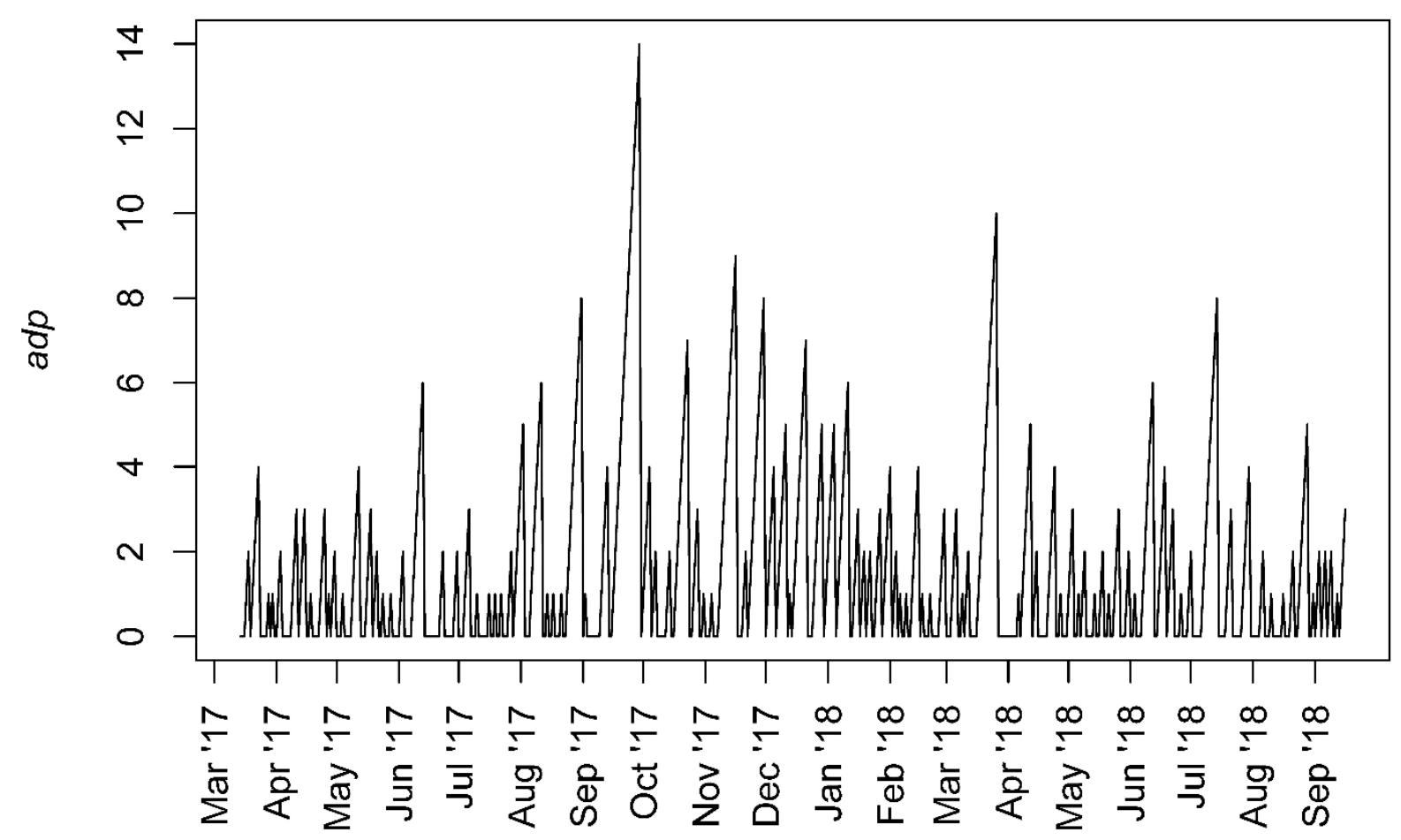

Figure S18: Temporal profile of daily antecedent dry period ( $a d p)$ in days.

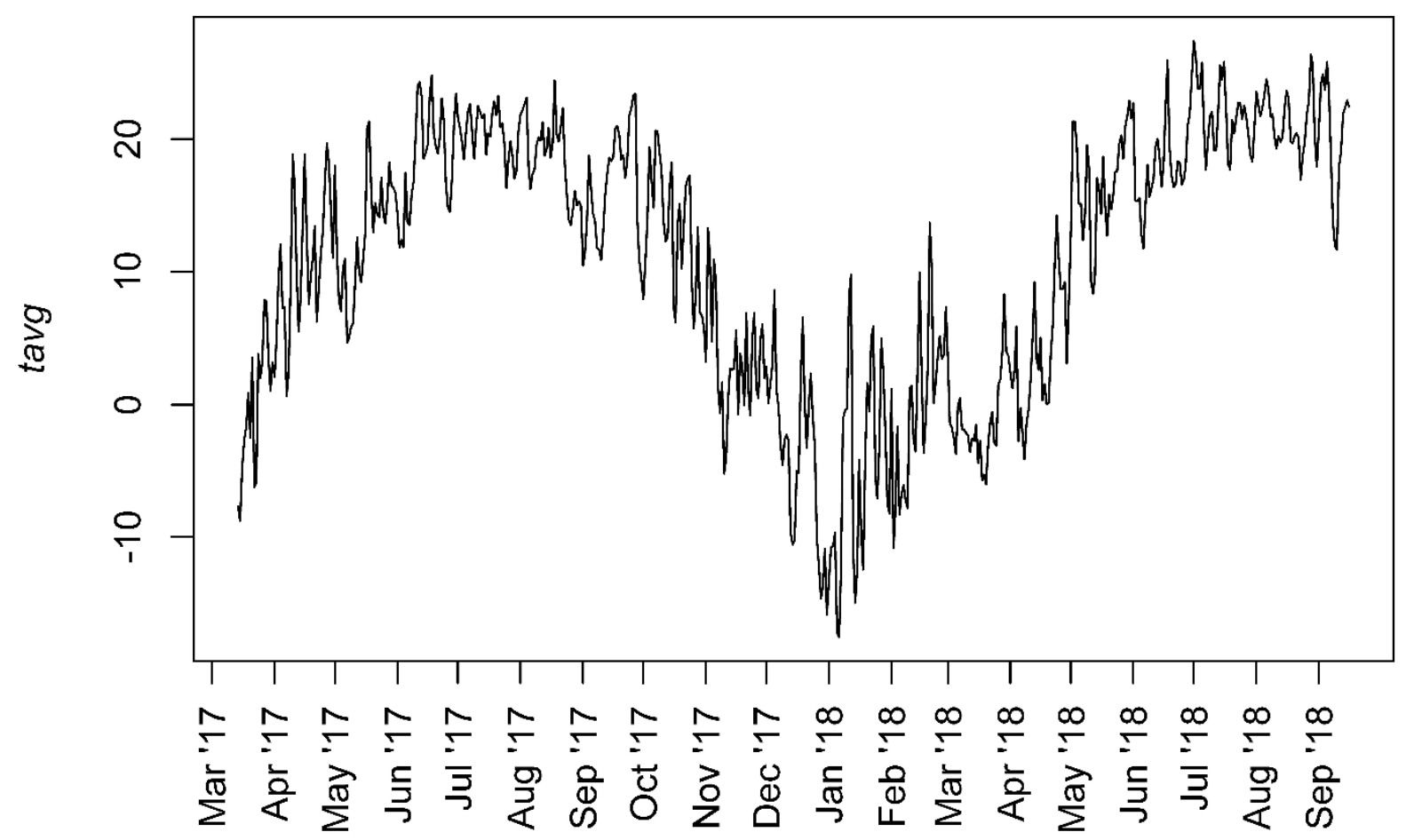

Figure S19: Temporal profile of average daily air temperature $(\operatorname{tavg})$ in ${ }^{\circ} \mathrm{C}$. 


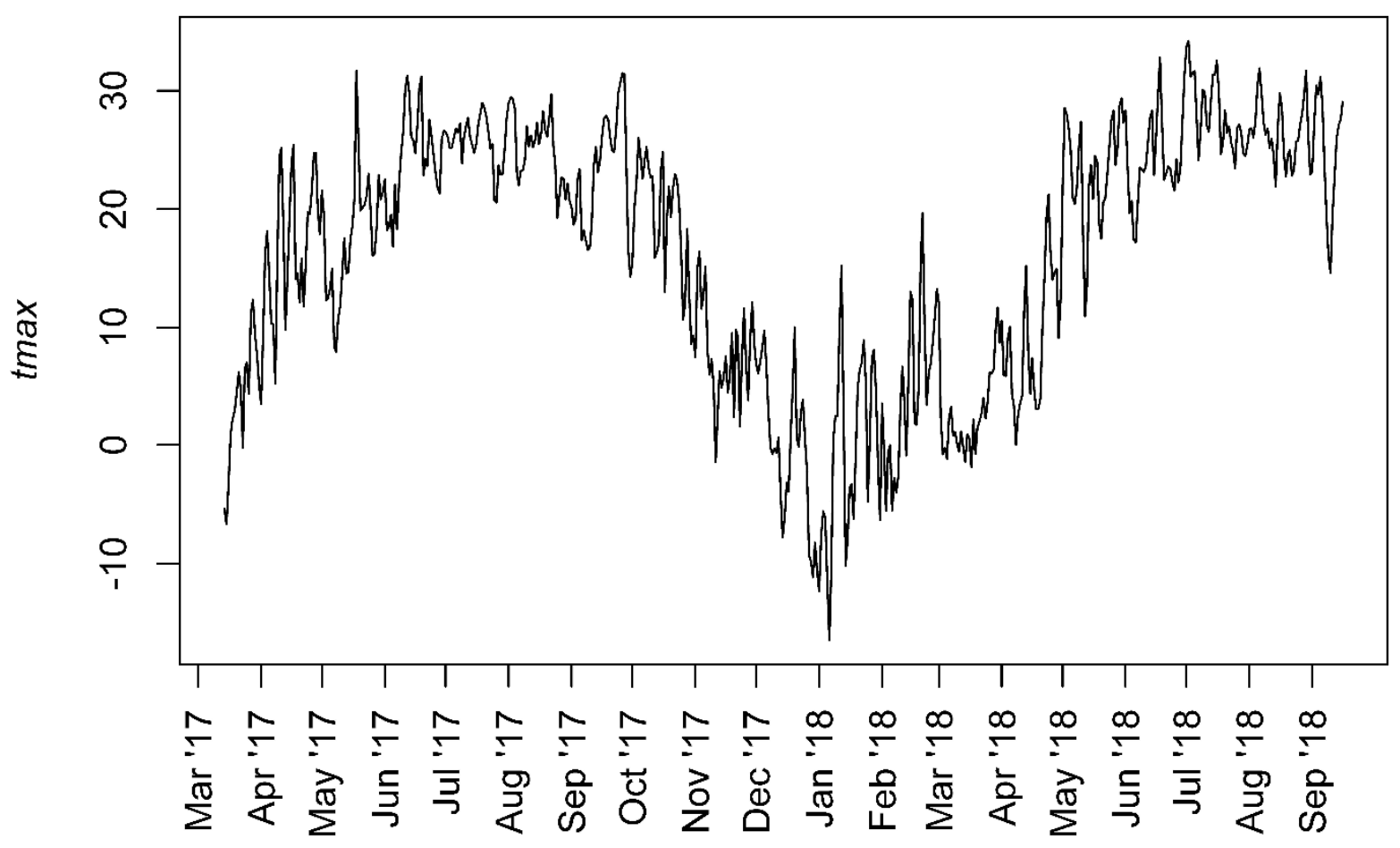

Figure S20: Temporal profile of average maximum daily air temperature $(\operatorname{tmax})$ in ${ }^{\circ} \mathrm{C}$.

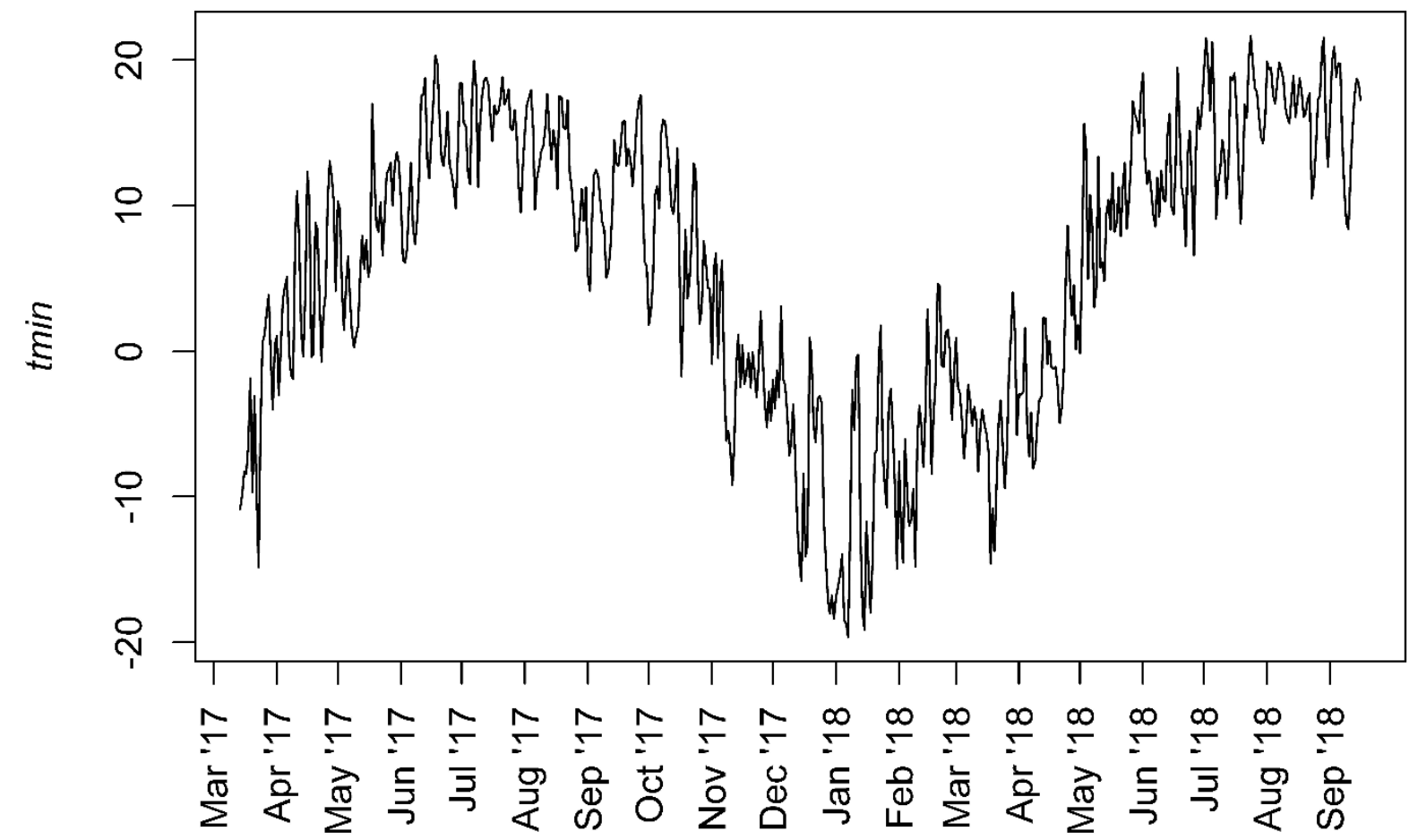

Figure S21: Temporal profile of average minimum daily air temperature $($ tmin $)$ in ${ }^{\circ} \mathrm{C}$. 


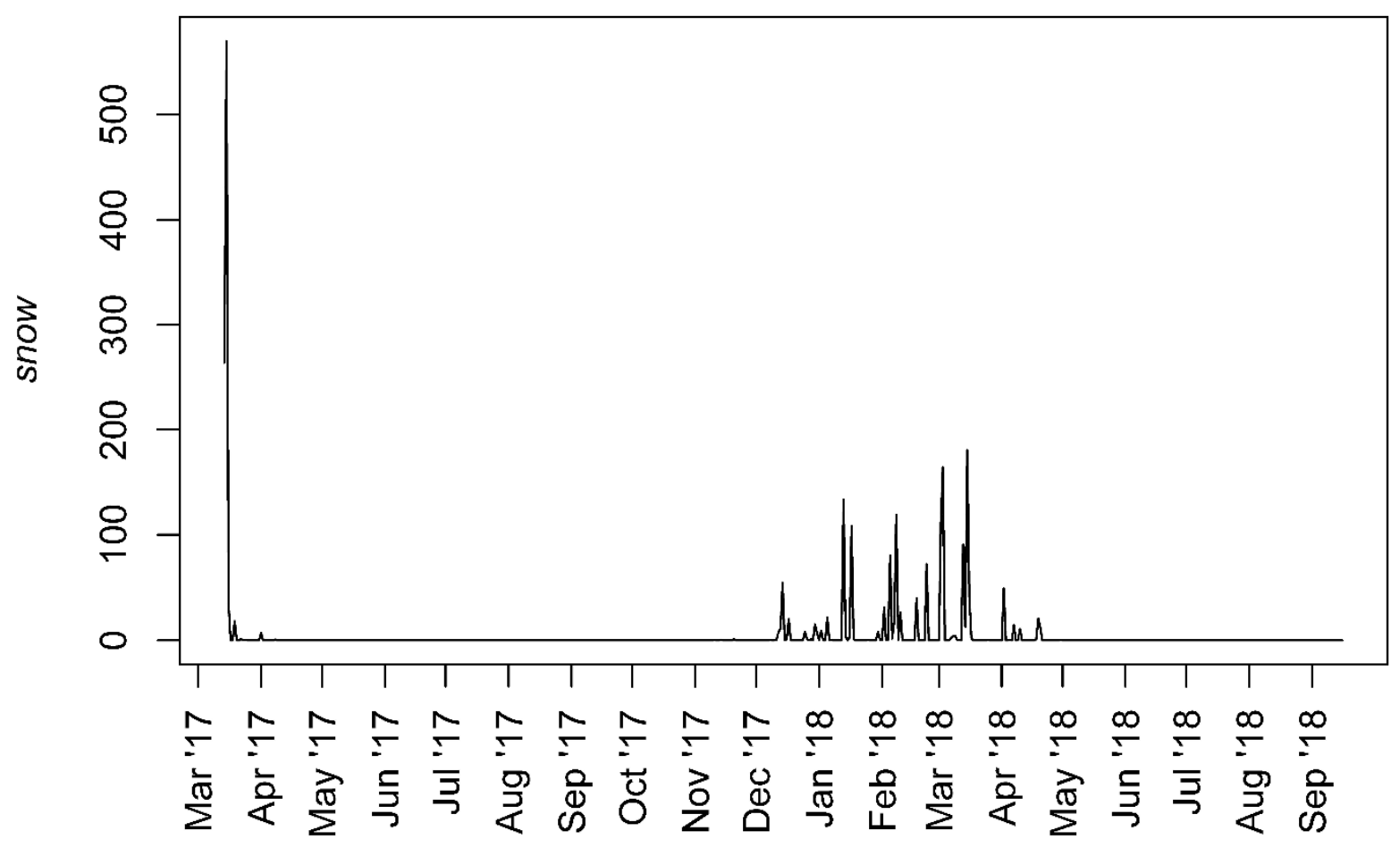

Figure S22: Temporal profile of average daily snowfall (snow) in mm.

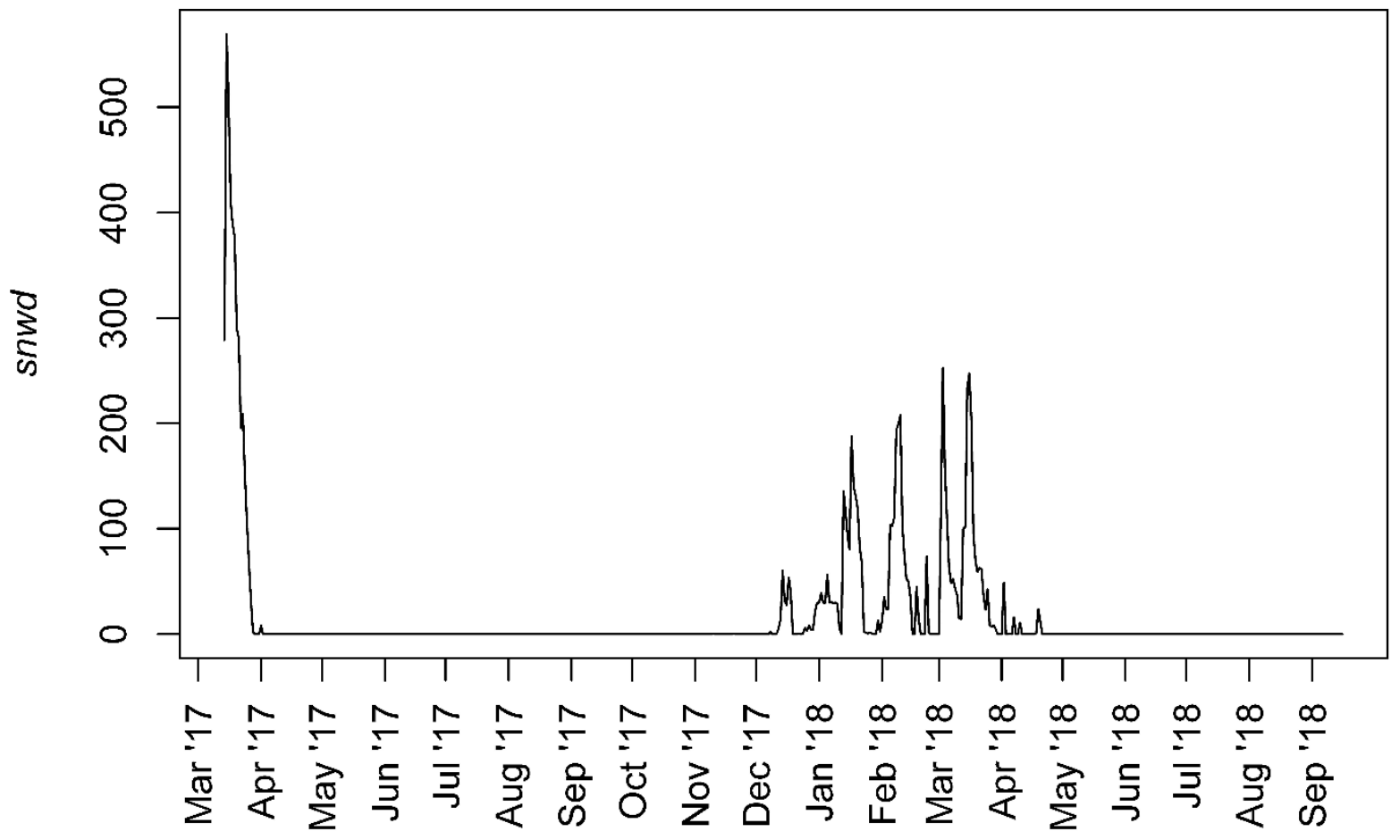

Figure S23: Temporal profile of average daily snow depth (snwd) in $\mathrm{mm}$. 


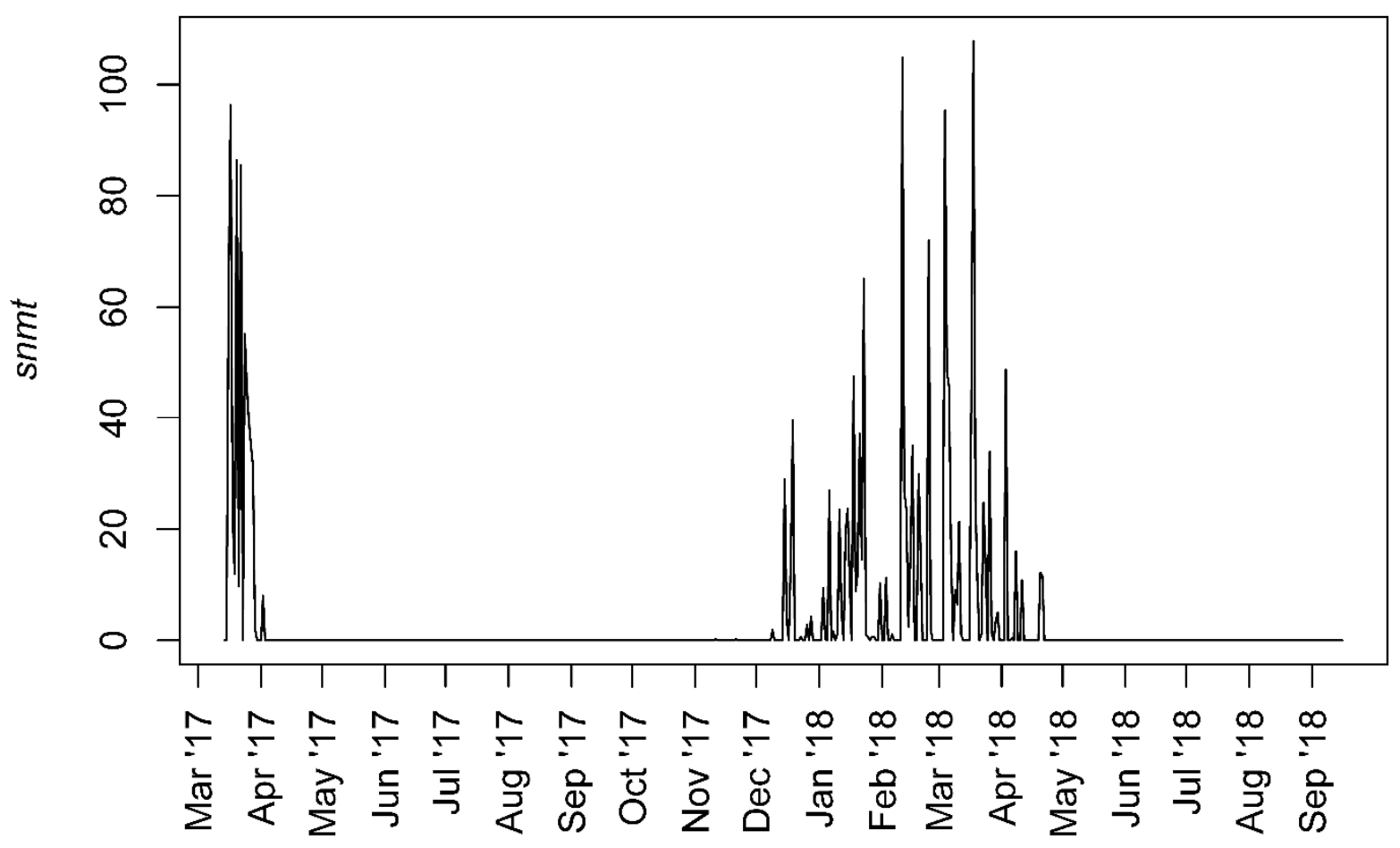

Figure S24: Temporal profile of average daily snow melt (snmt) in mm.

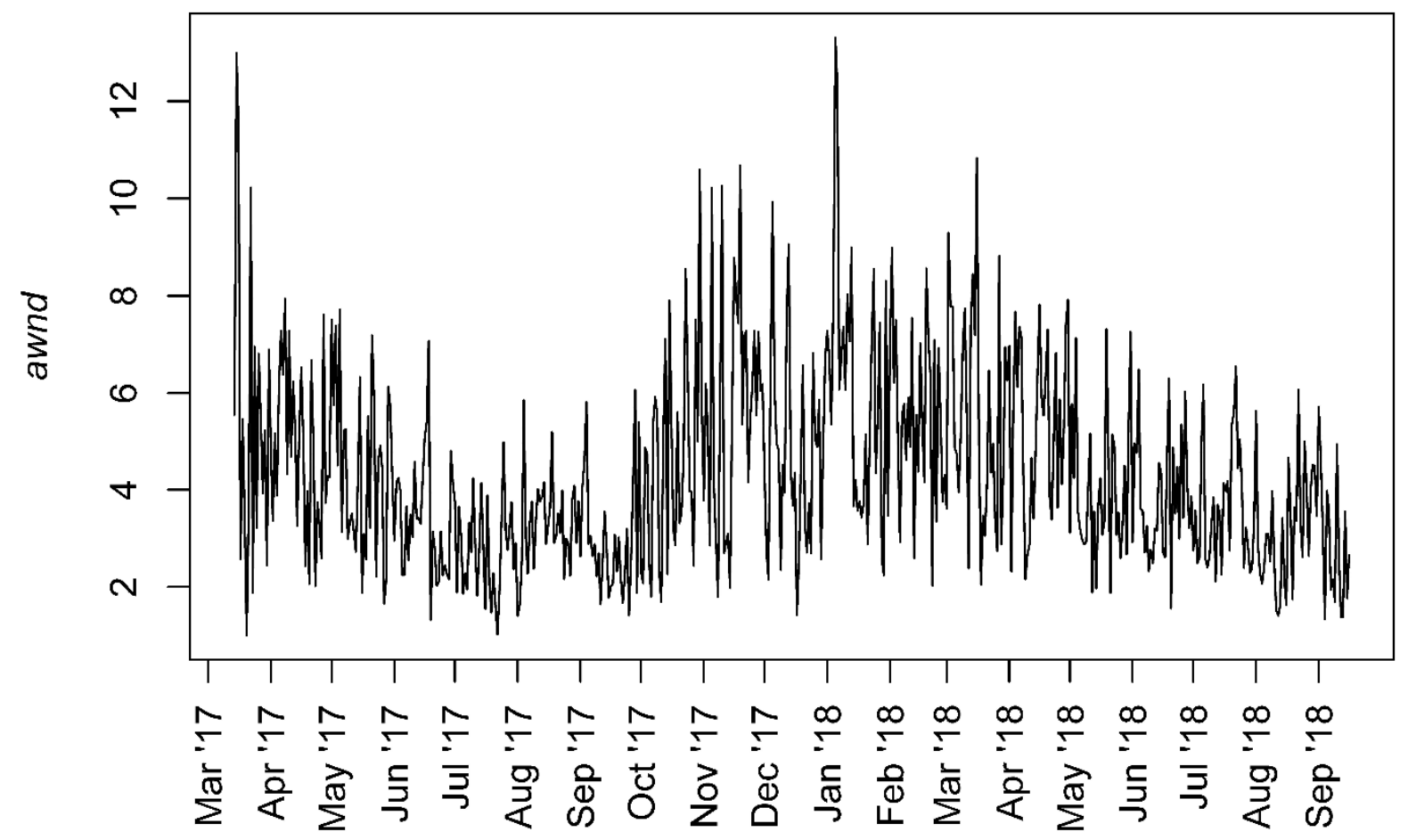

Figure S25: Temporal profile of average daily wind speed (awnd) in $\mathrm{m} \cdot \mathrm{s}^{-1}$. 


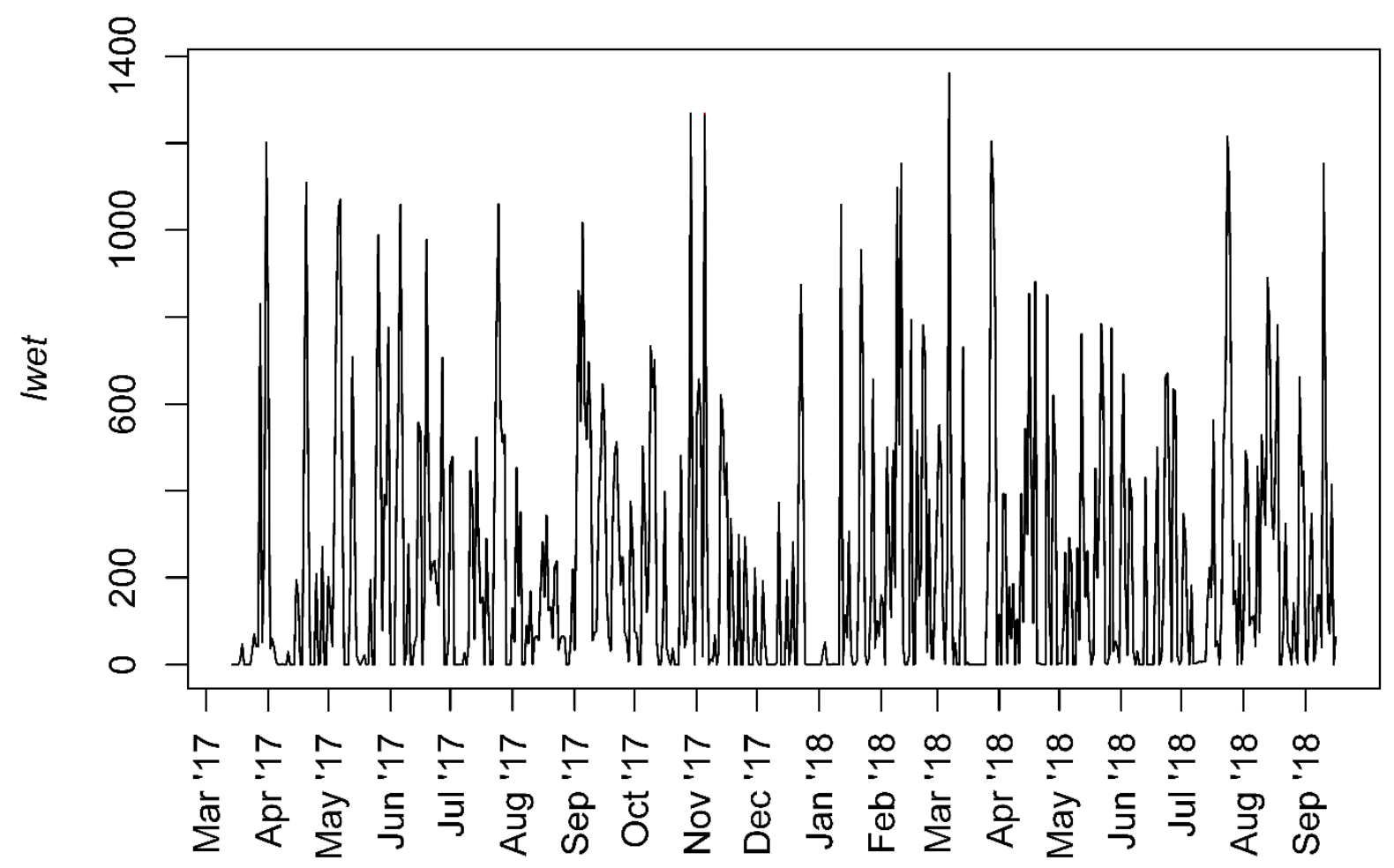

Figure S26: Temporal profile of total daily leaf wetness (lwet) in min.

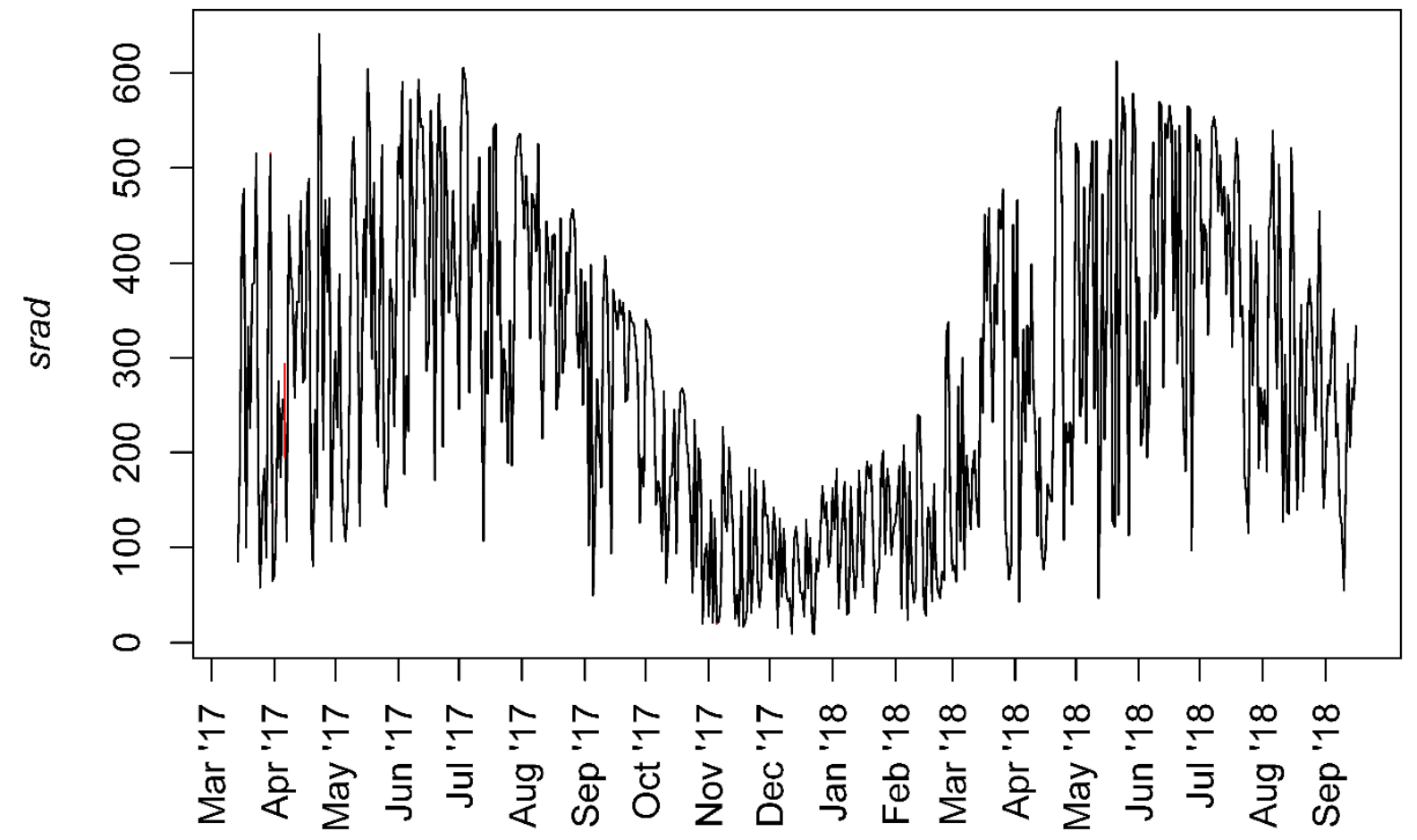

Figure S27: Temporal profile of total daily solar radiation ( $\mathrm{srad}$ ) in langleys. 


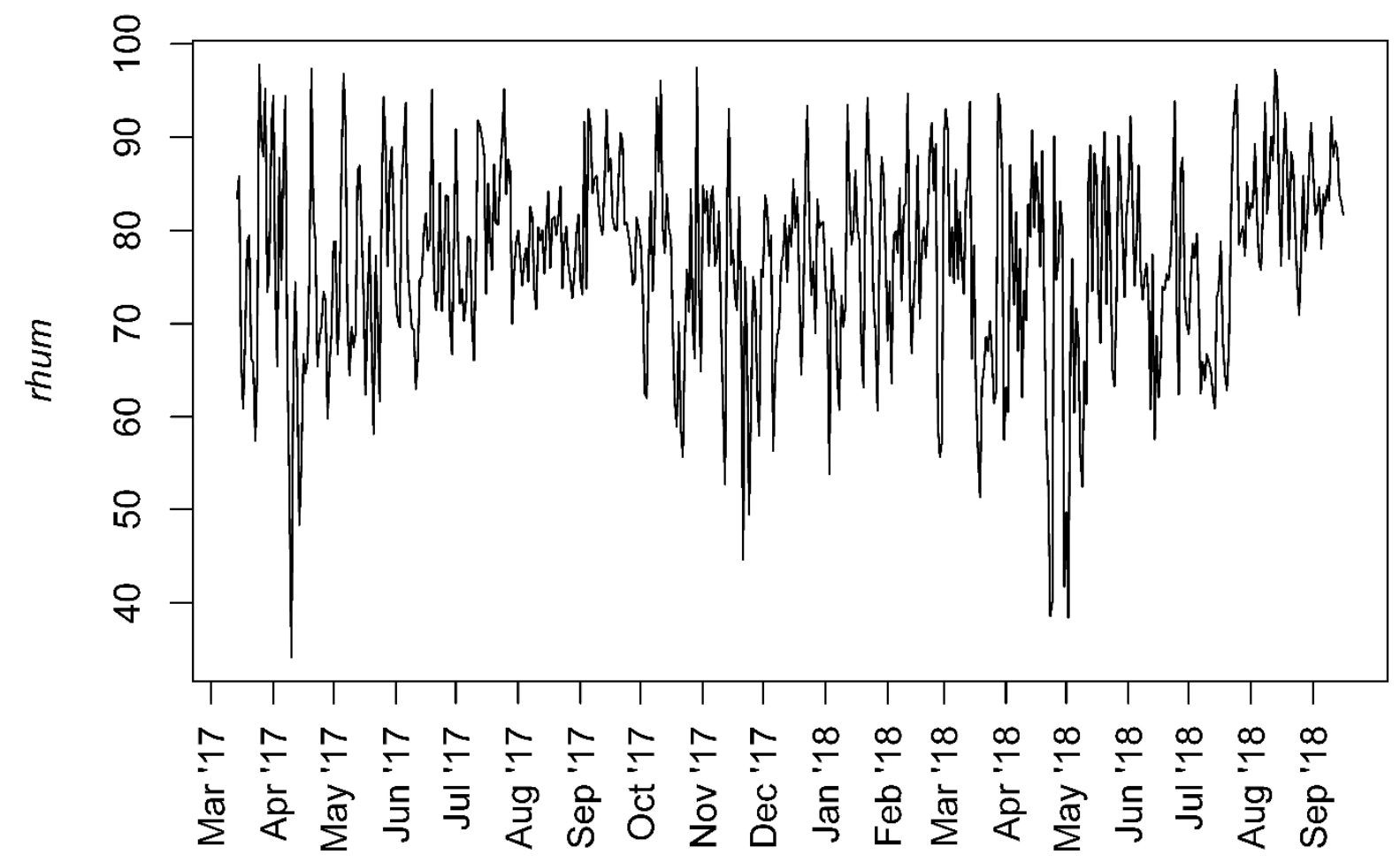

Figure S28: Temporal profile of average daily relative humidity (rhum) in \%.

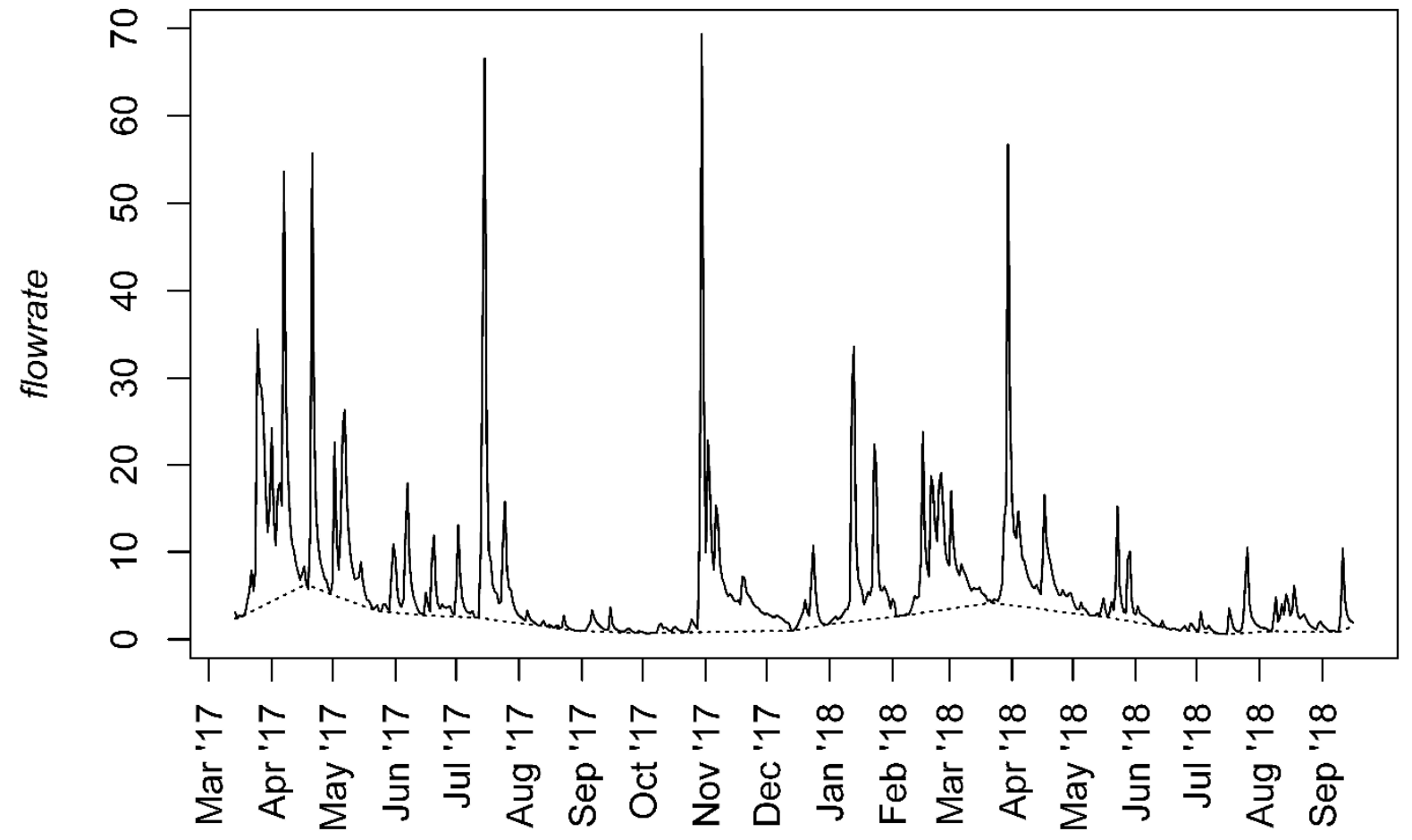

Figure S29: Temporal profile of average daily streamflow rate (flow; solid line) and baseflow rate (dotted line) in $\mathrm{m}^{3} \cdot \mathrm{s}^{-1}$. 


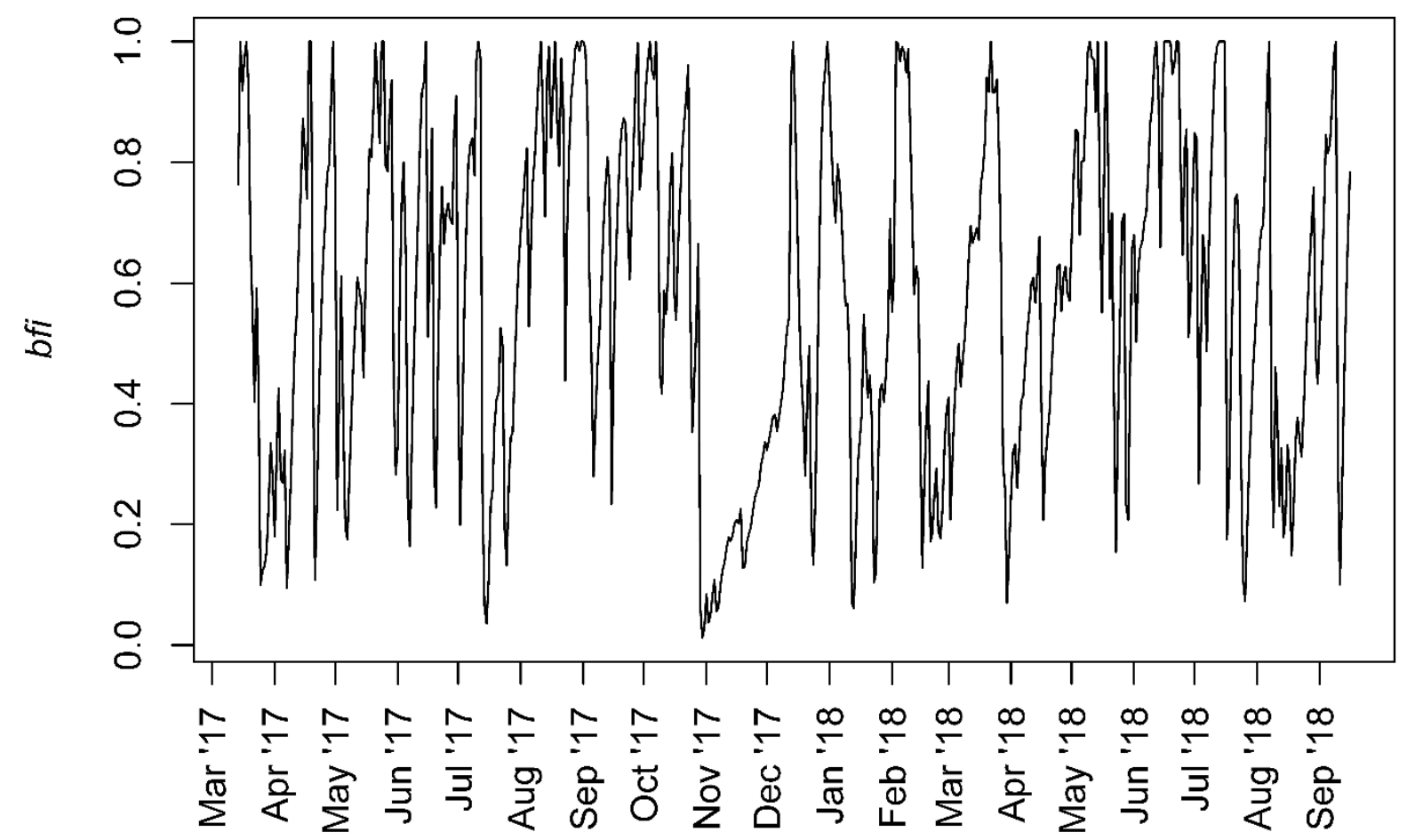

Figure S30: Temporal profile of average daily baseflow index (bfi).

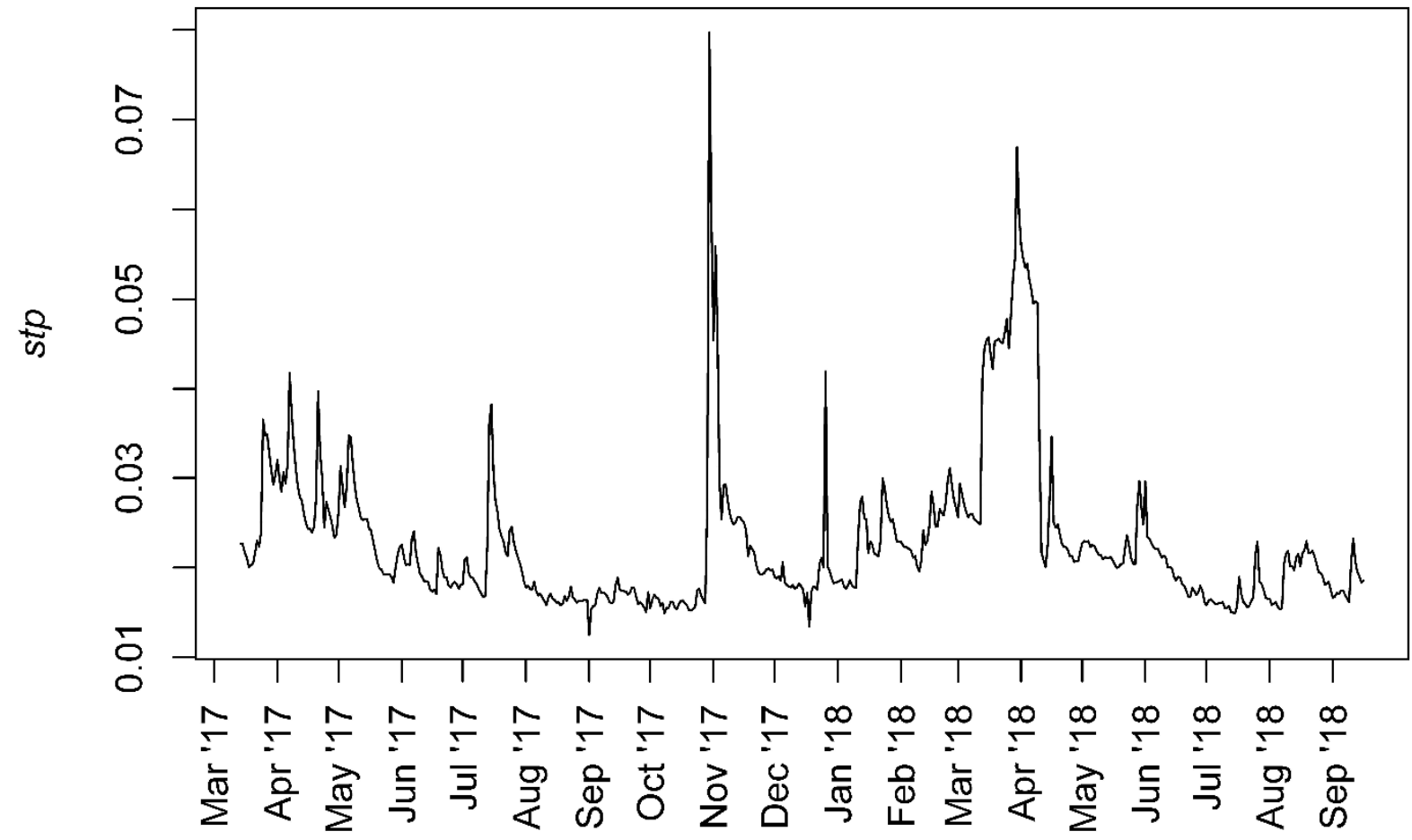

Figure S31: Temporal profile of average daily sewage treatment plant discharge $(s t p)$ in $\mathrm{m}^{3} \cdot \mathrm{s}^{-1}$. 


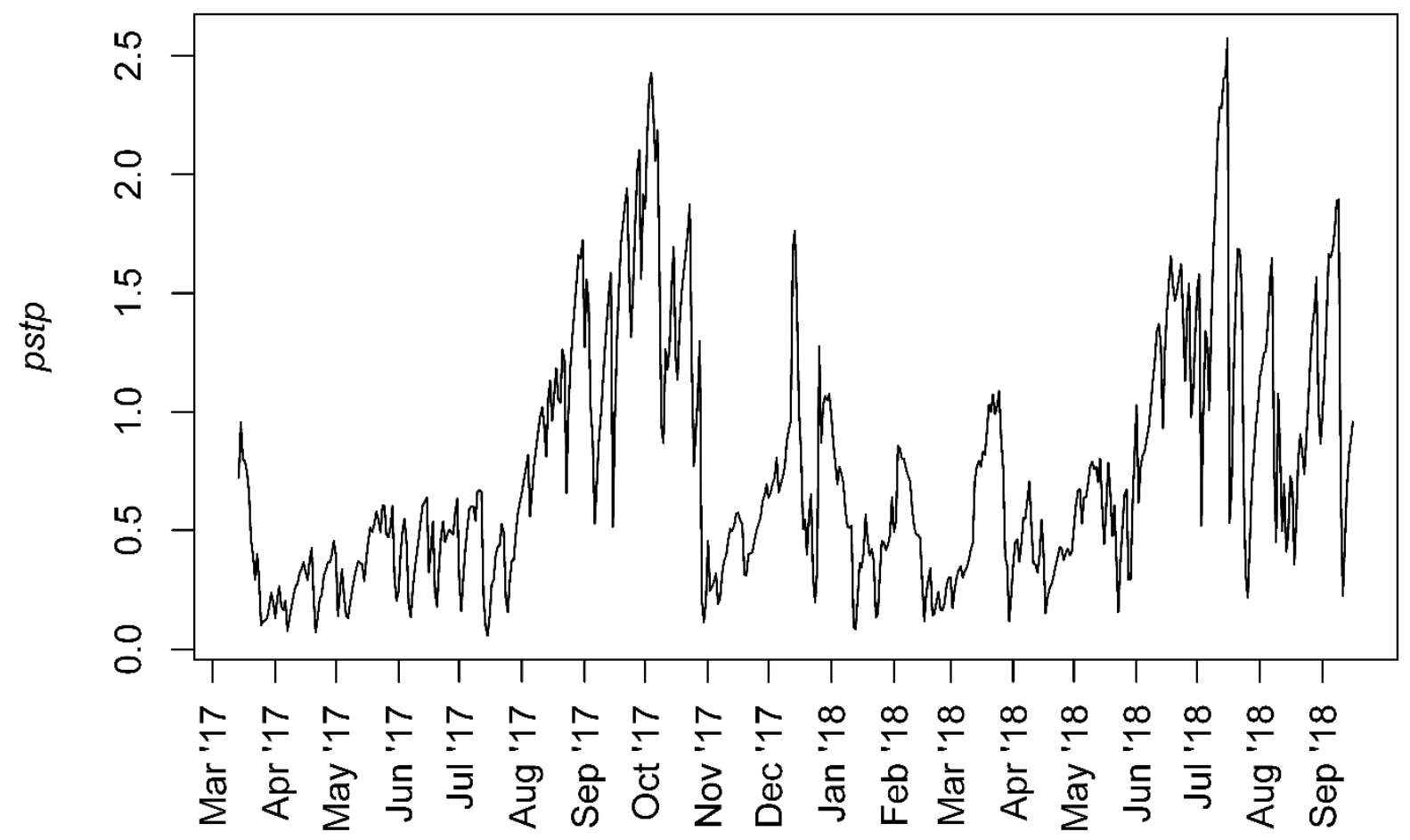

Figure S32: Temporal profile of average daily sewage proportion (pstp) in \%.

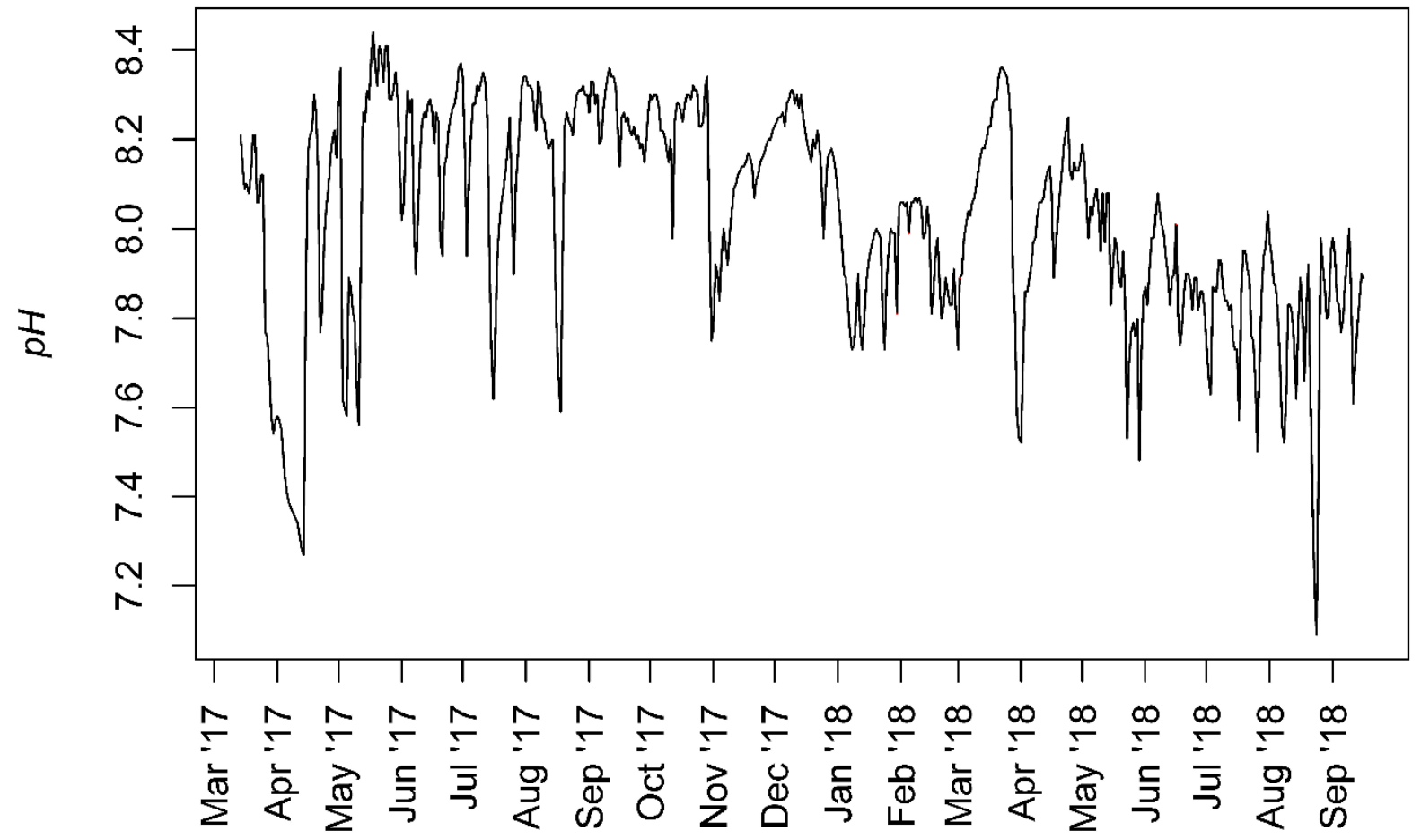

Figure S33: Temporal profile of average daily $\mathrm{pH}(p H)$. 


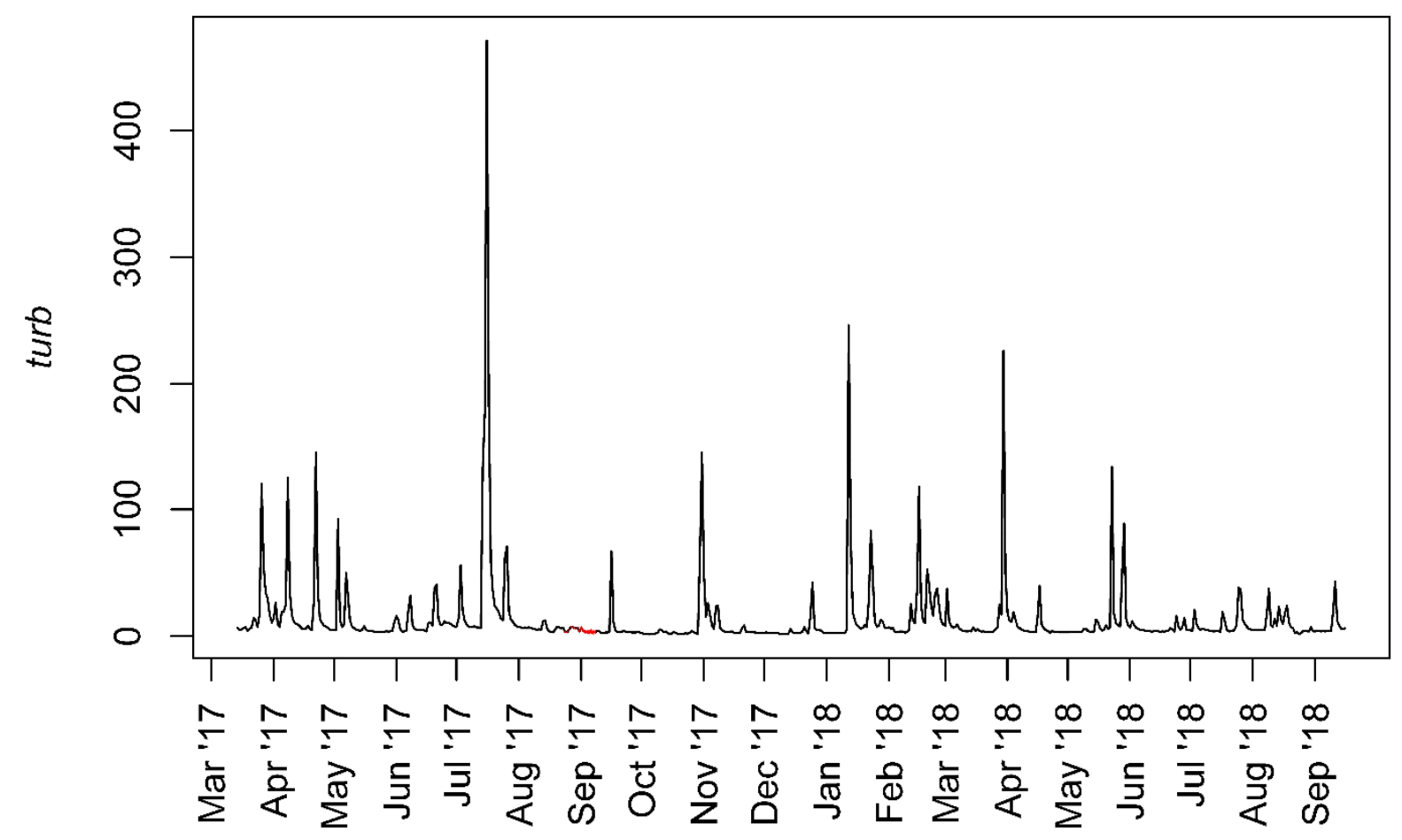

Figure S34: Temporal profile of average daily turbidity (turb) in NTU.

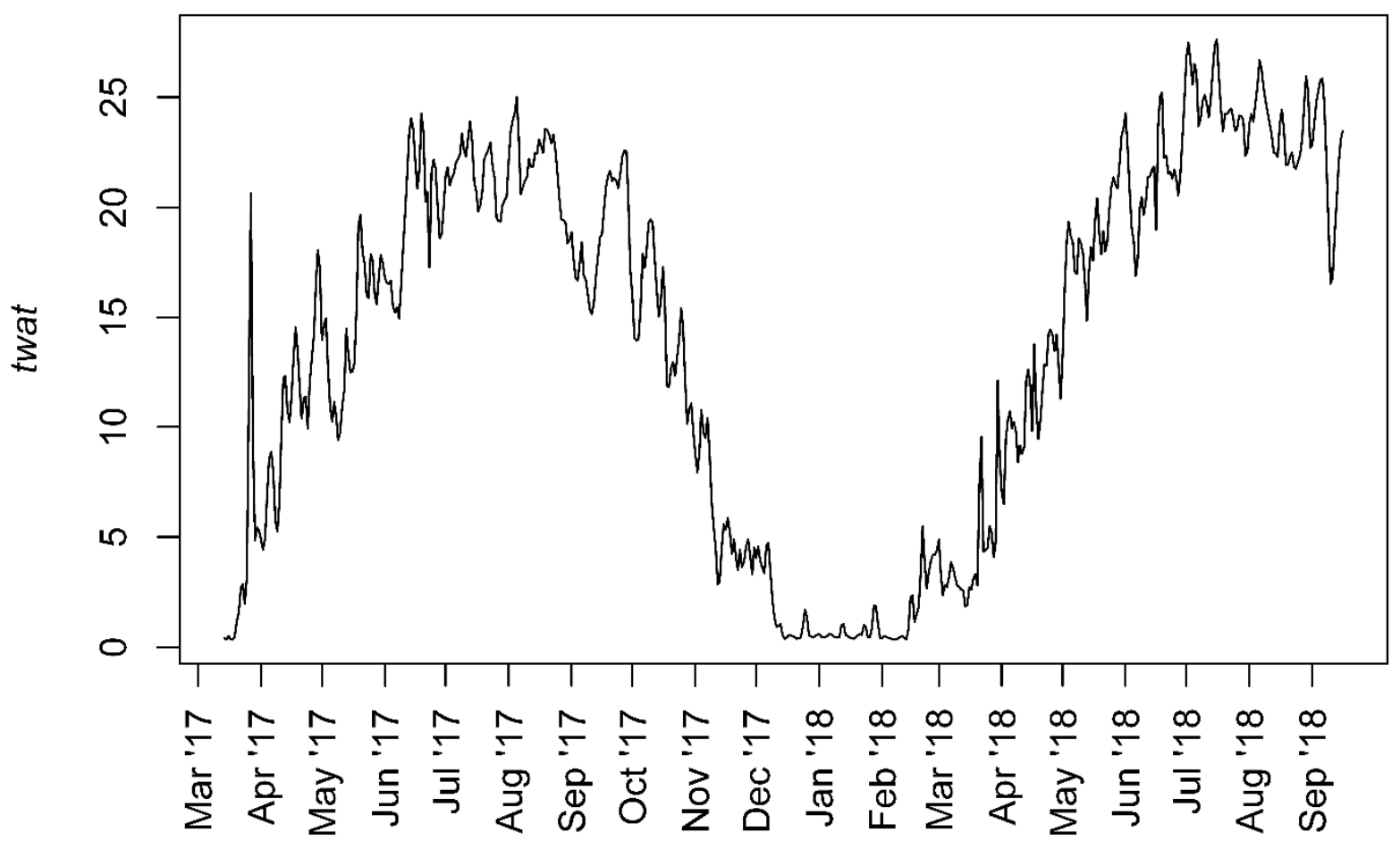

Figure S35: Temporal profile of average daily water temperature (twat) in ${ }^{\circ} \mathrm{C}$. 


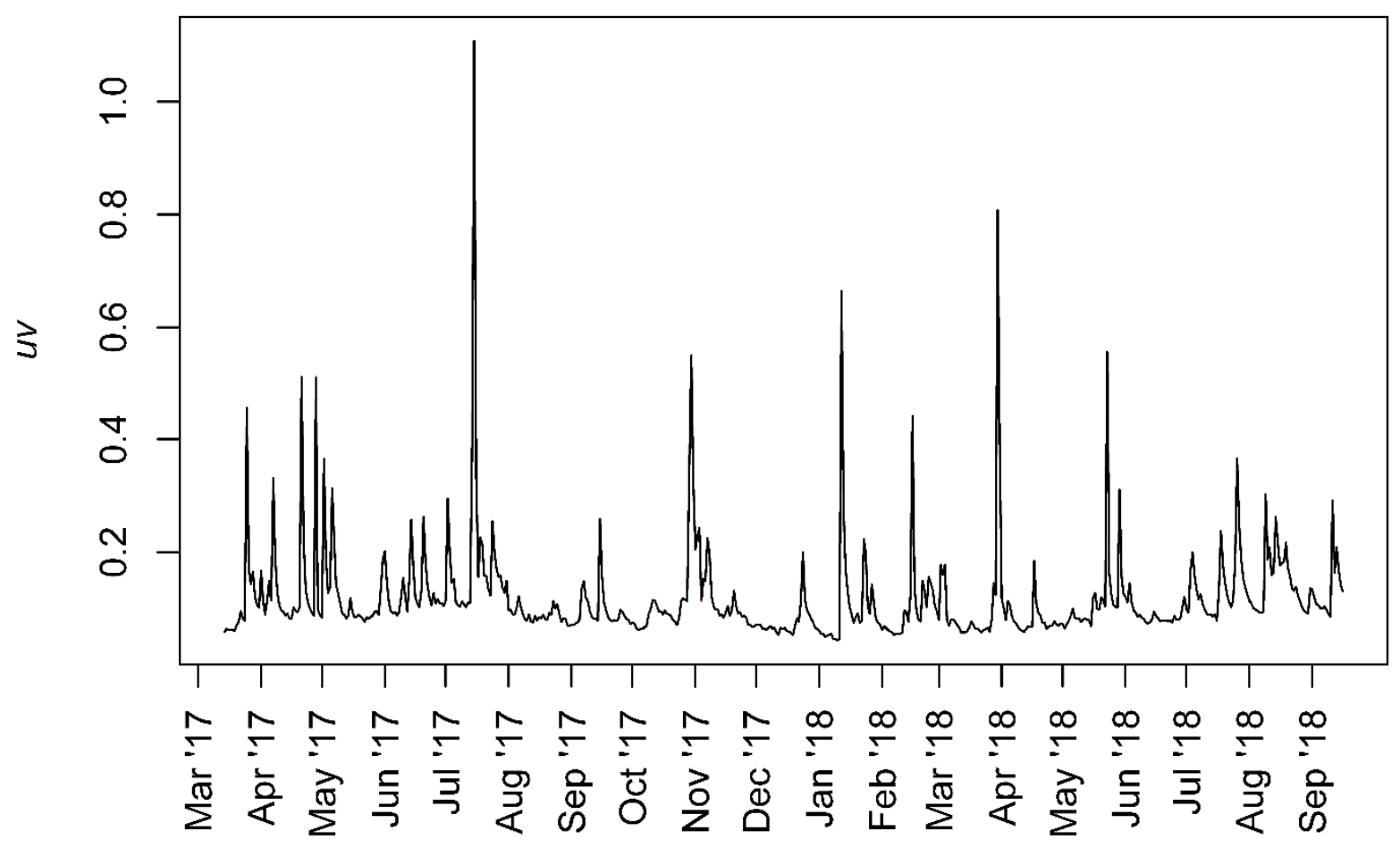

Figure S36: Temporal profile of average daily $\mathrm{UV}_{254}$ absorbance $(u v)$.

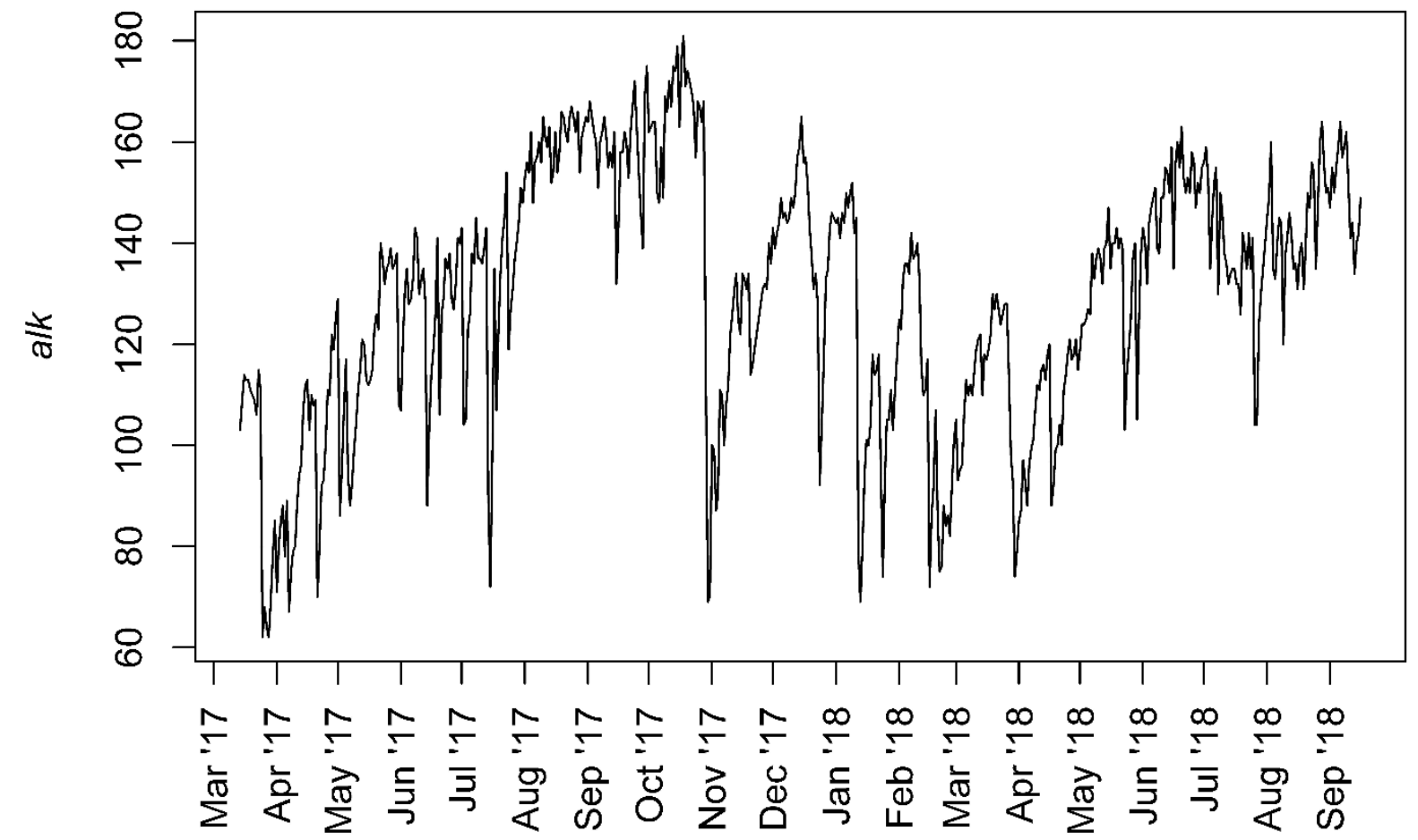

Figure S37: Temporal profile of daily alkalinity measurements (alk) in $\mathrm{mg} \cdot \mathrm{L}^{-1}$ as $\mathrm{CaCO}_{3}$. 


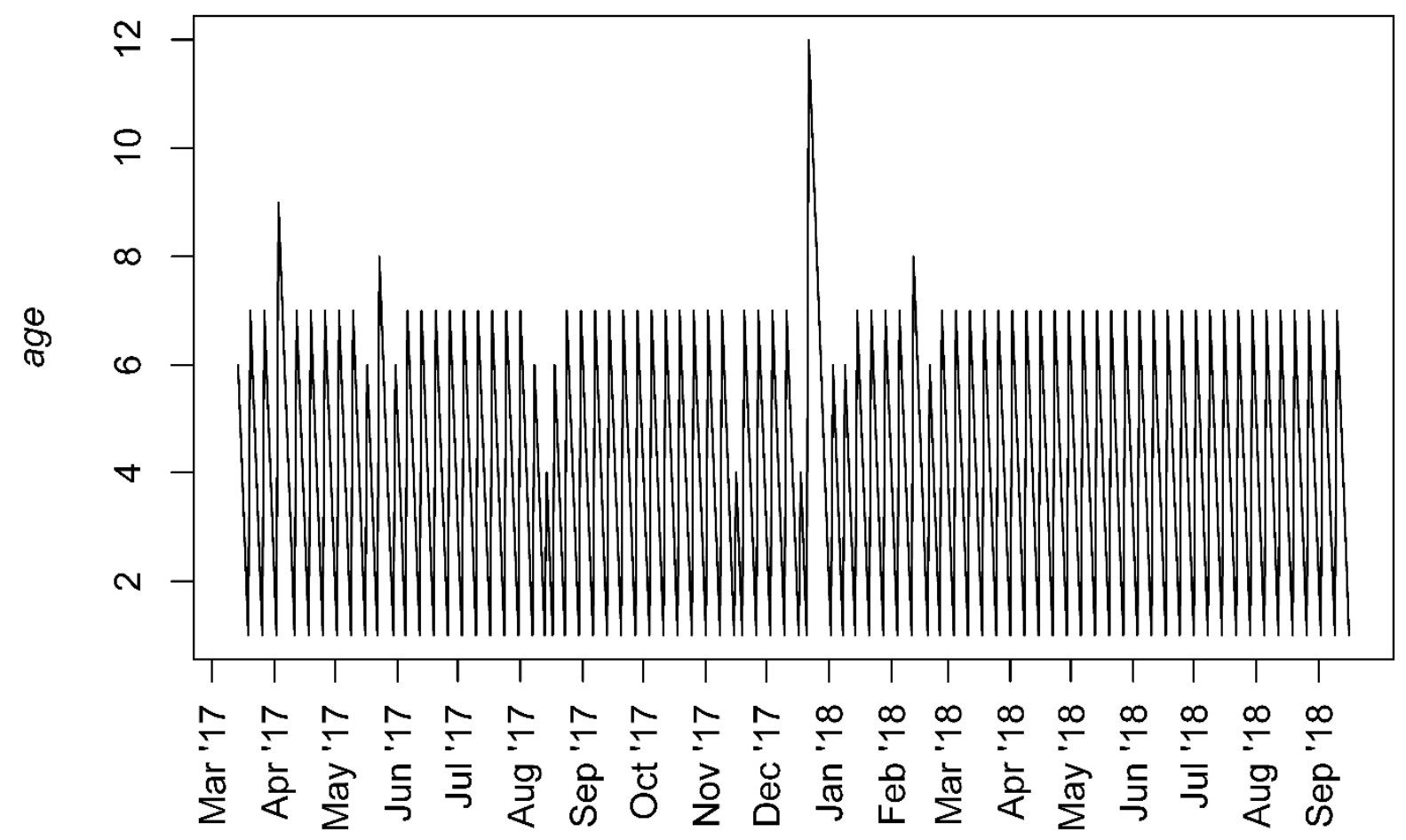

Figure S38: Temporal profile of average daily water sample age (age) in days. 
Figure S39 describes the optimization of the apparent growing seasons for ATR. For each growing season (2017 and 2018), the cross-correlation coefficients between ATR concentrations and $b f i$ were examined along assumed growing seasons that varied by start date and duration. Each point on the figure describes the correlation coefficient for an assumed growing season. Optimal apparent growing seasons were selected by maximizing both the correlation coefficients and the duration of the apparent growing season. Based on our results, $b f i$ is only well-correlated with the agriculture-derived micropollutants during their respective apparent growing seasons.

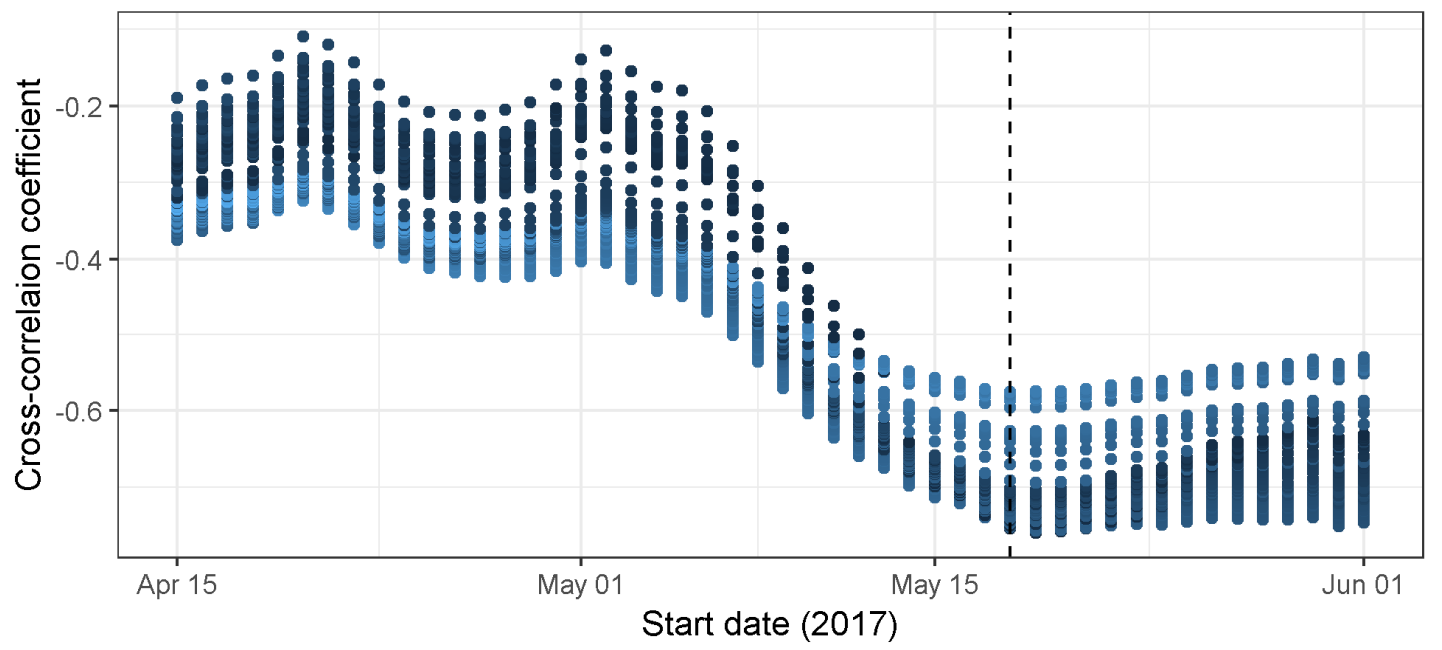

\section{Duration} (days)
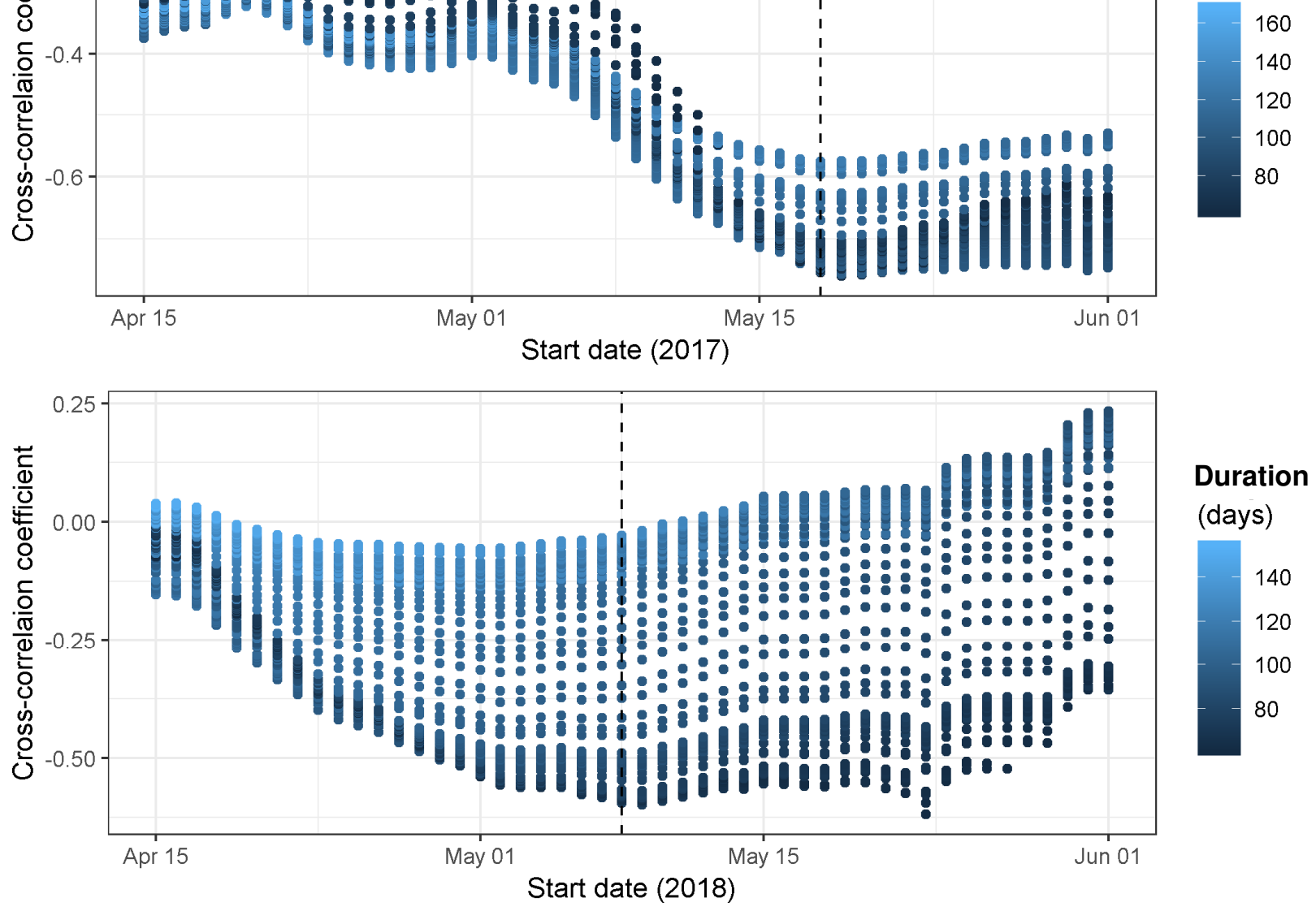

Figure S39: Optimal growing seasons for atrazine as determined by correlation with $b f i$. Optimal start dates are marked with a dashed line. 


\section{Multivariable regression}

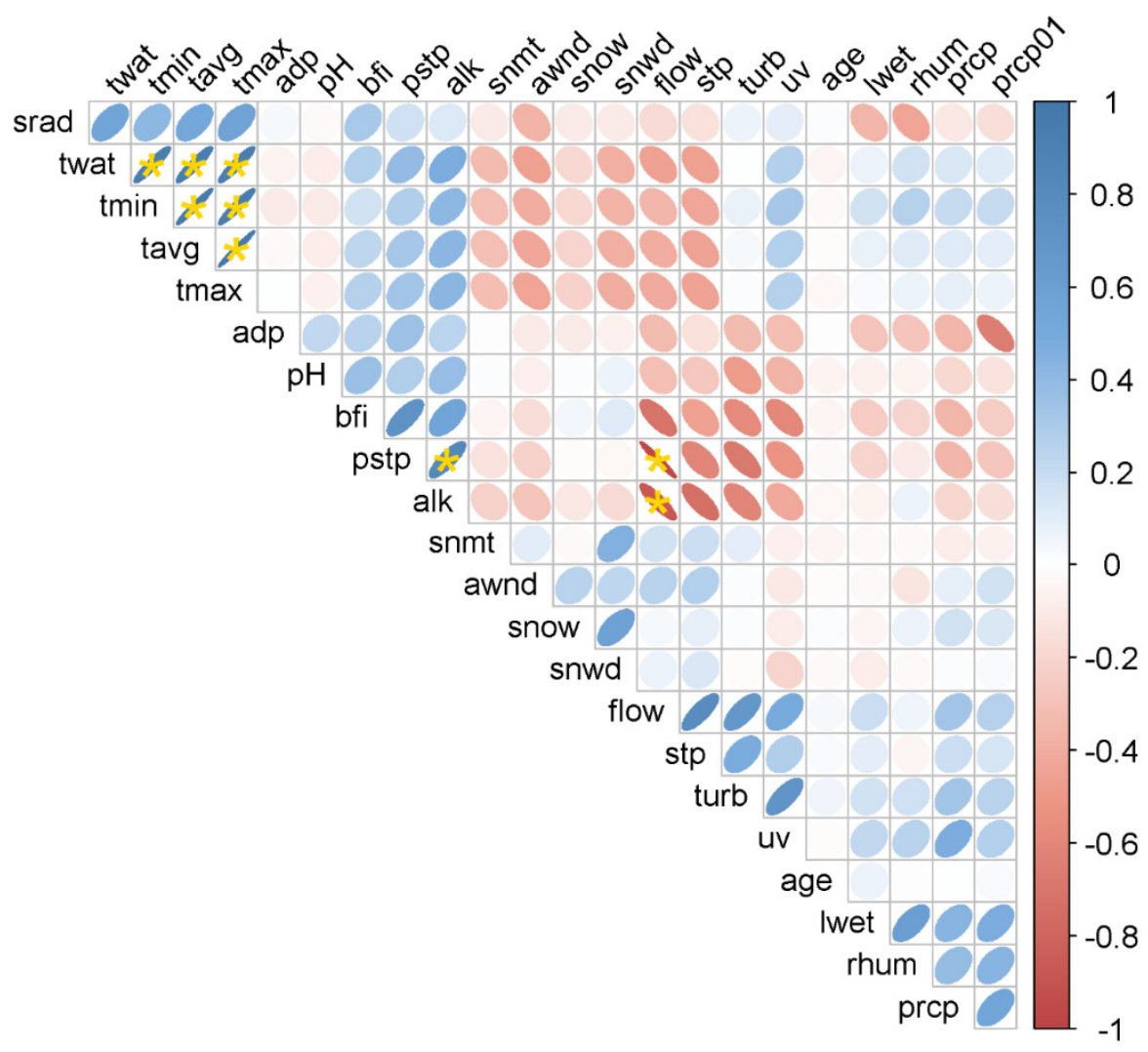

Figure S40: Environmental covariates (average of the imputed datasets) correlation matrix. Significant Pearson correlations $(\rho>0.8$, p-value $<0.01)$ are marked $(*)$.

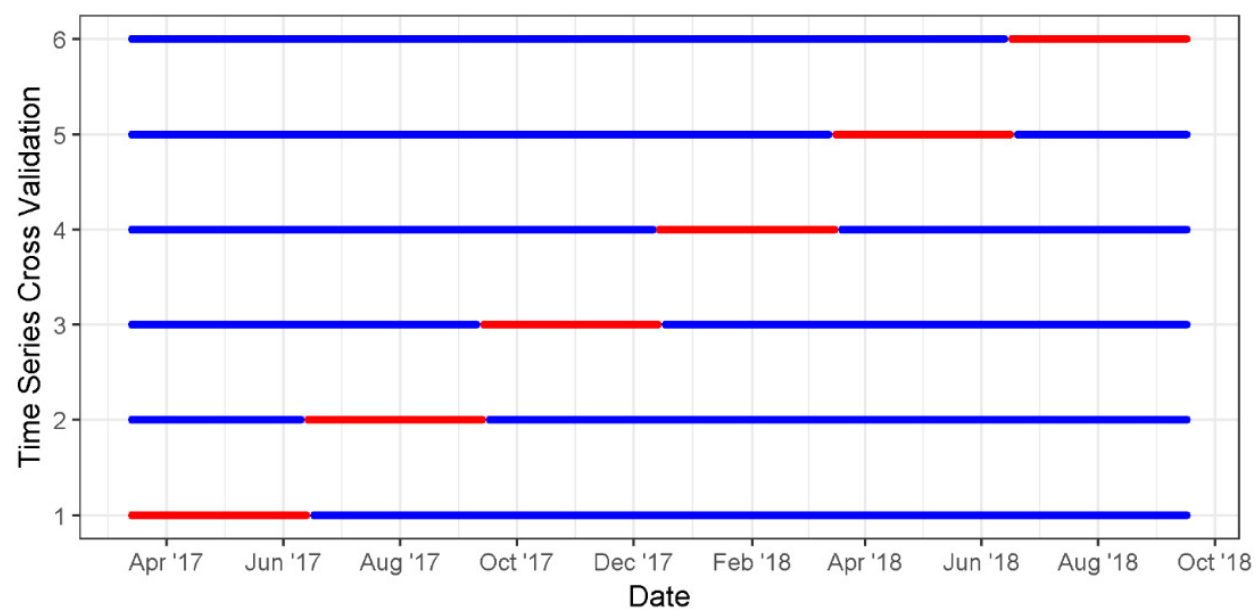

Figure S41: Cross-validation time series; blue lines represent training sets and red lines represent test sets. 
Figures S42 - S61 describe the bivariate regression plots and significant final standardized coefficients for the generalized least squares (GLS) and logistic (logit) regressions following the model framework outlined in the main text. The bivariate regression plots include all environmental covariates in the best subsets regression model equations for each micropollutant profile. The interaction terms between grow and the environmental covariates are specified as grow_X and non_grow_X. Each imputed dataset is plotted at 20\% opacity (non-imputed points are solid, while imputed points are semitransparent) and best fit lines (red) for each imputed dataset are shown. Logit regression plots show the density histograms for peak events (top, red) and nonpeak events (bottom, blue). The final standardized coefficient plots represent the best combination of environmental covariates based on corrected Akaike information criterion (AICc) and multimodel inference. The boxplots represent the coefficients determined for each cross validation sample set for each imputed dataset $(n=30)$, and the blue points represent the overall mean coefficient value (error bars represent the $95 \%$ confidence interval). Train and test set model diagnostics (GLS, adjusted $\mathrm{R}^{2}$; logit, accuracy) are provided for each micropollutant profile. 
Atrazine (ATR)
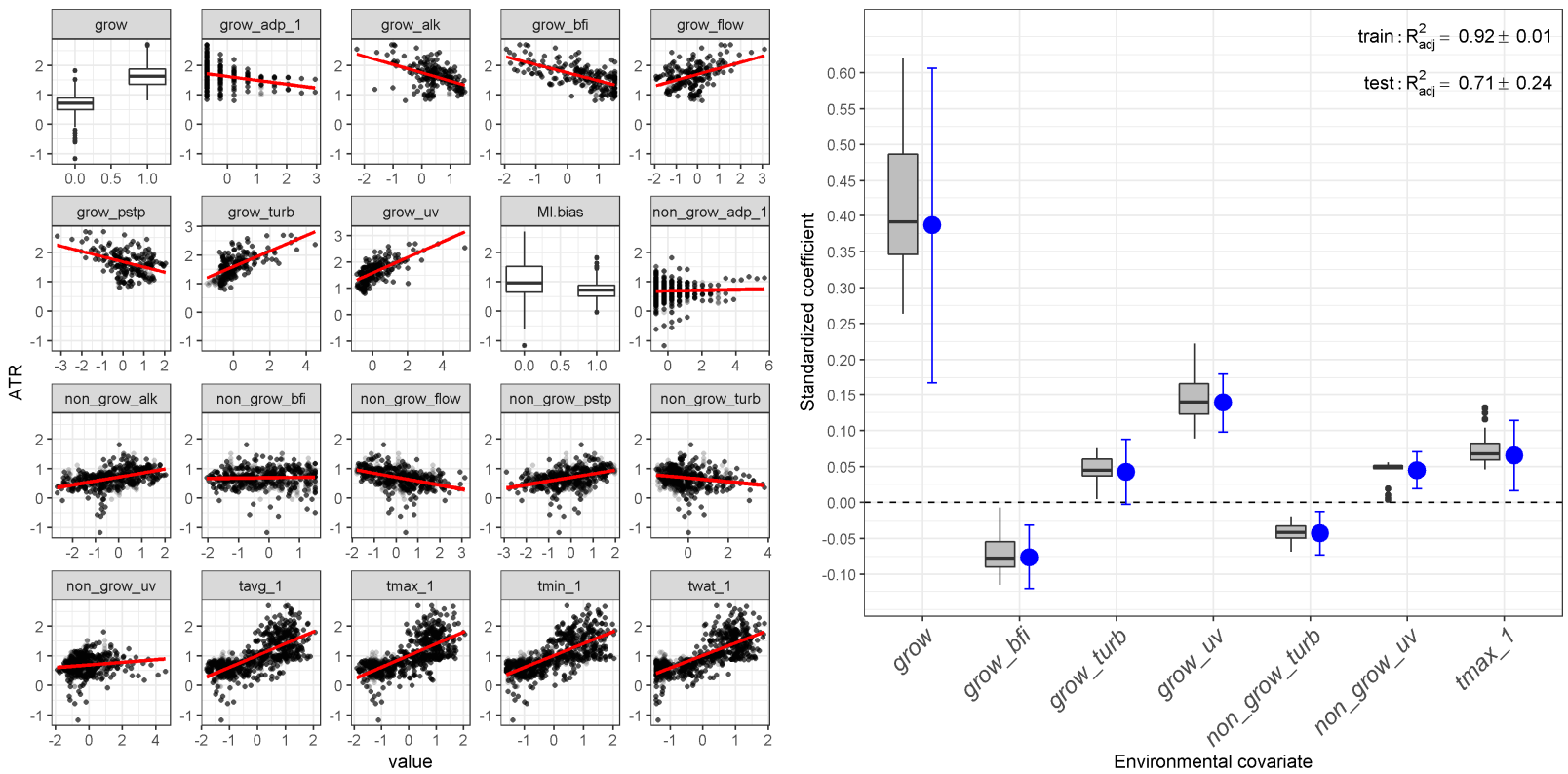

Figure S42: Bivariate GLS regression plots for ATR (left) and significant final standardized coefficients (right).
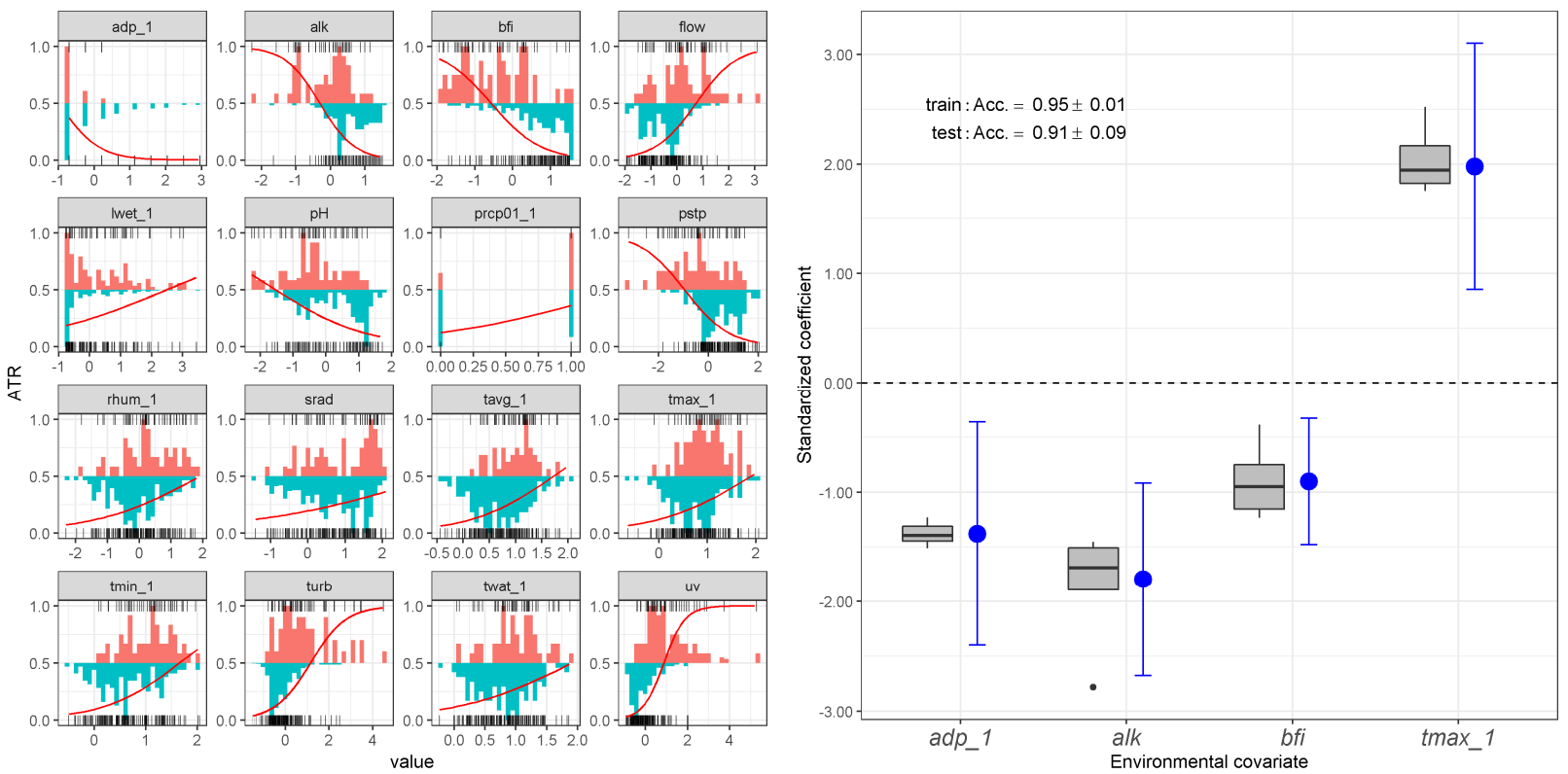

Figure S43: Bivariate logit regression plots for ATR (left) and significant final standardized coefficients (right) during the growing season. 
Atrazine-desethyl (ATR.d)
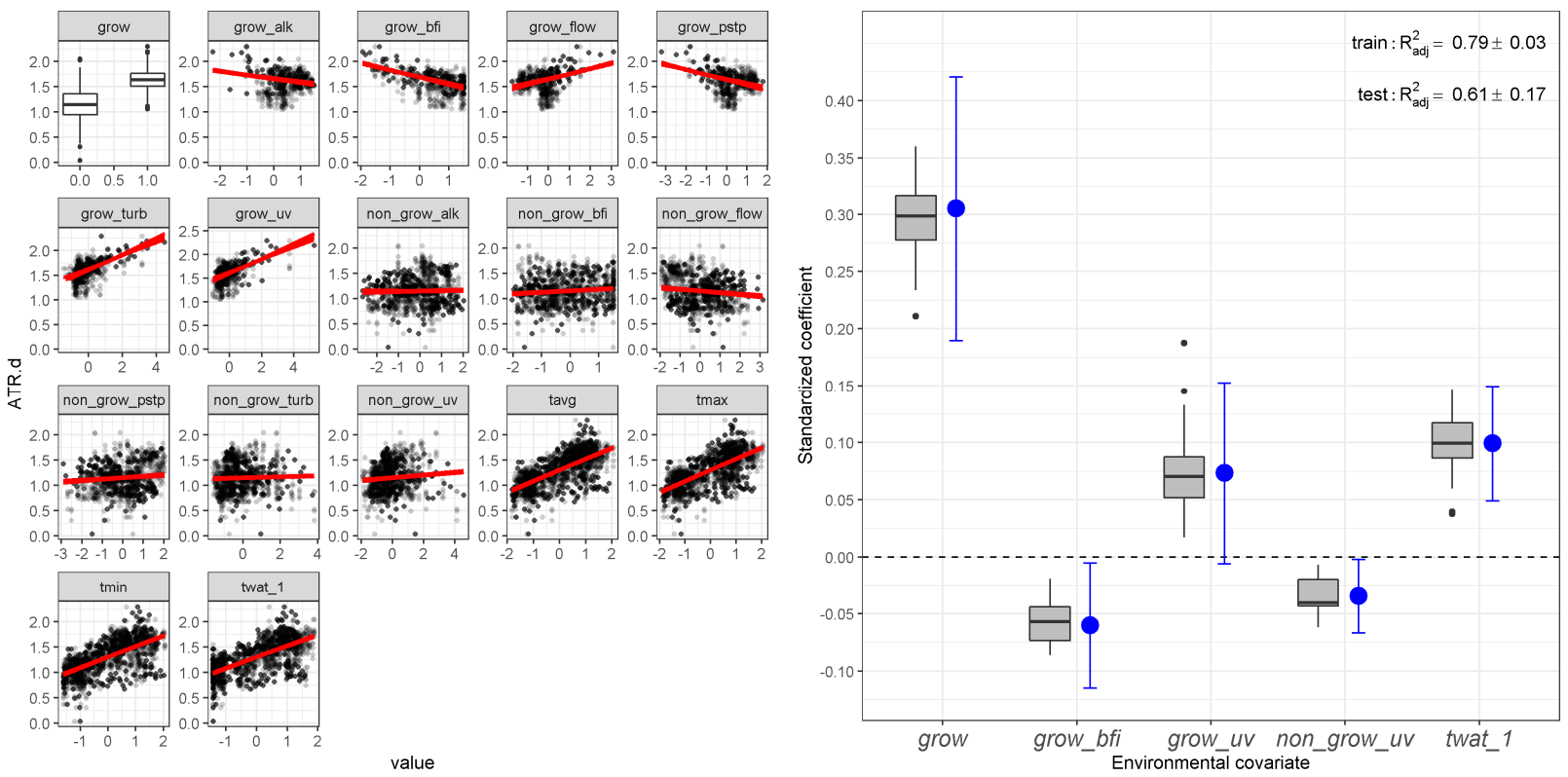

Figure S44: Bivariate GLS regression plots for ATR.d (left) and significant final standardized coefficients (right).
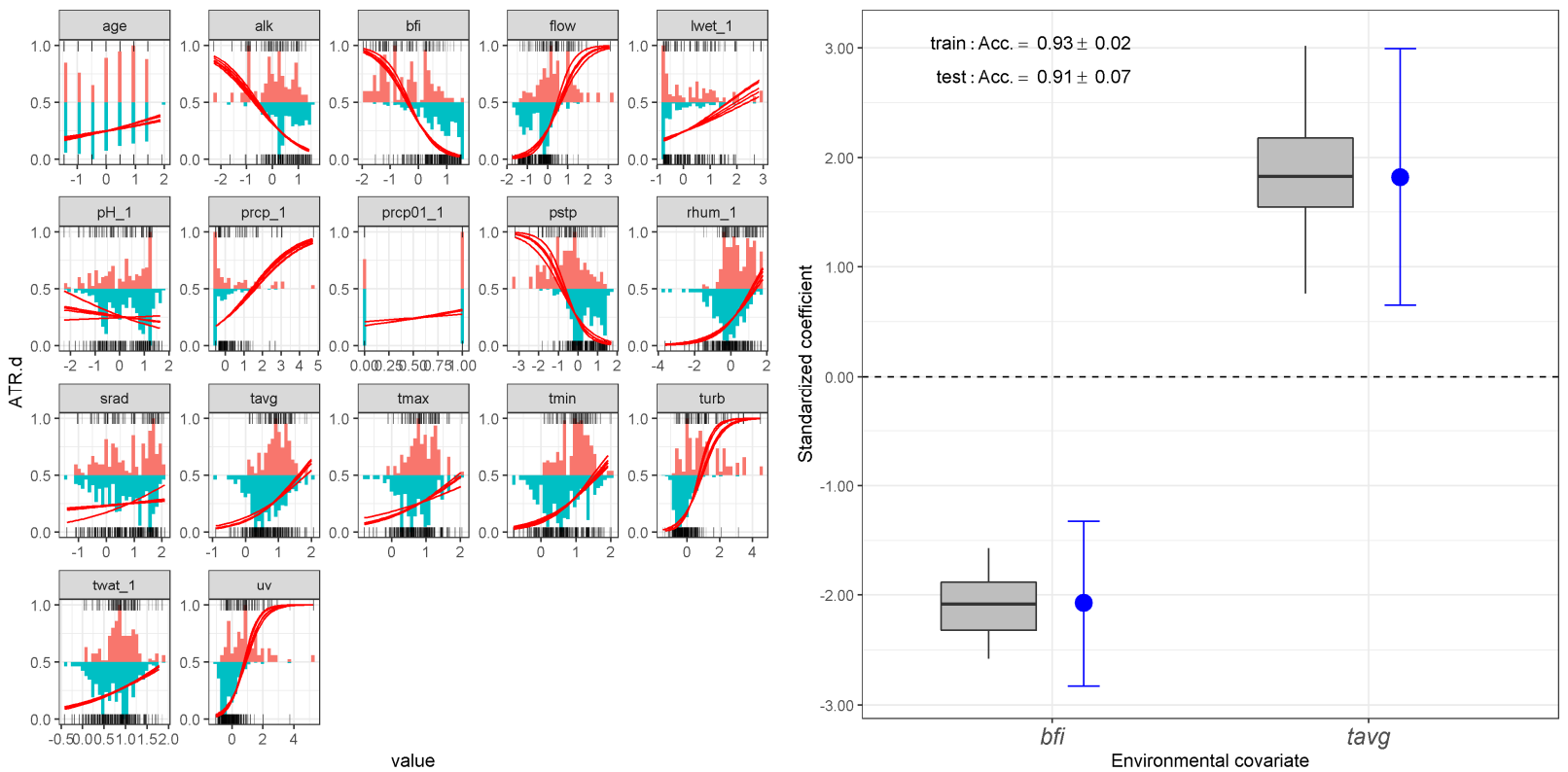

Figure S45: Bivariate logit regression plots for ATR.d (left) and significant final standardized coefficients (right) during the growing season. 


\section{Atrazine-2-hydroxy (ATR.h)}
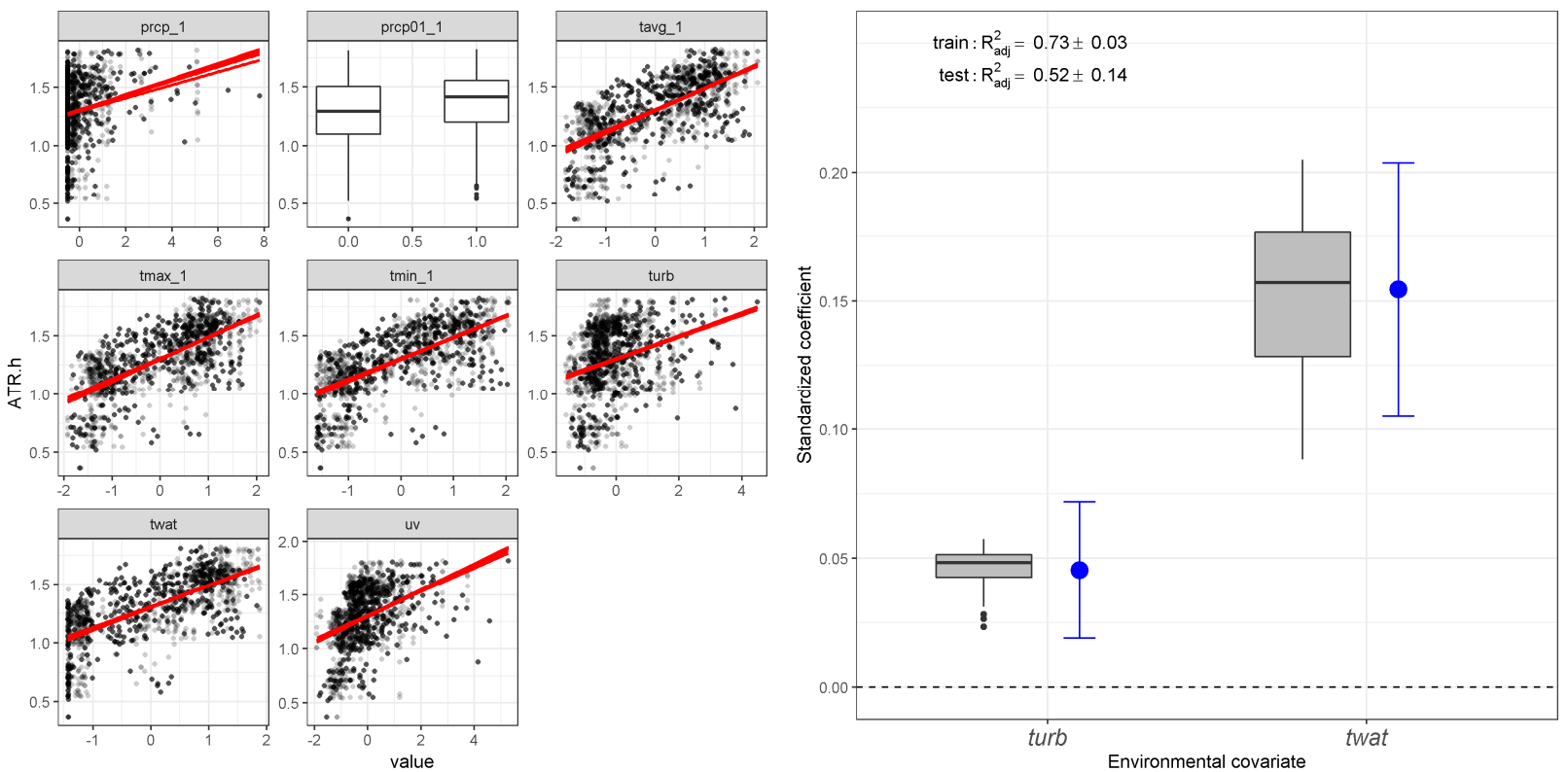

Figure S46: Bivariate GLS regression plots for ATR.h (left) and significant final standardized coefficients (right).
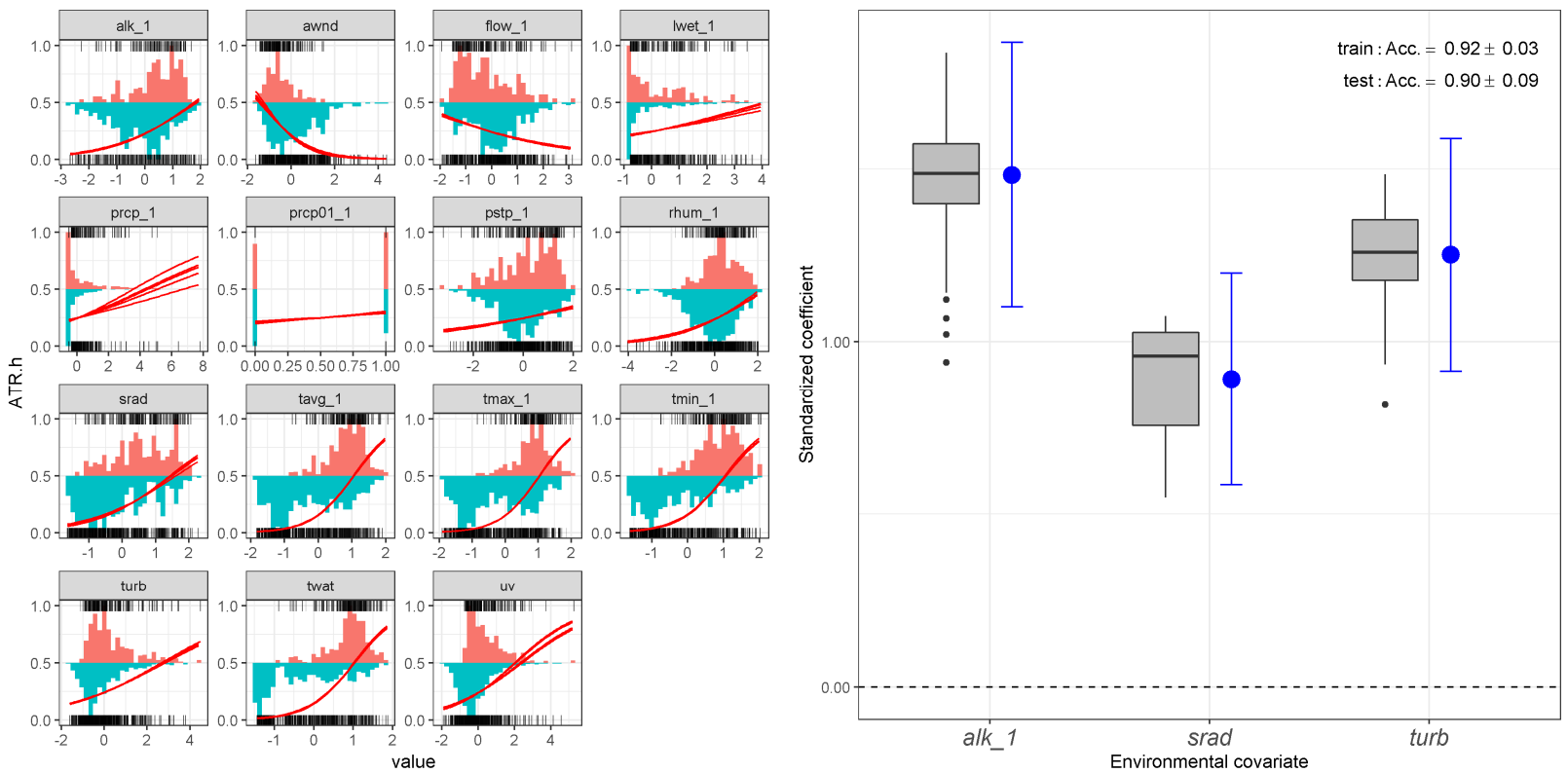

Figure S47: Bivariate logit regression plots for ATR.h (left) and significant final standardized coefficients (right). 


\section{Metolachlor (MET)}
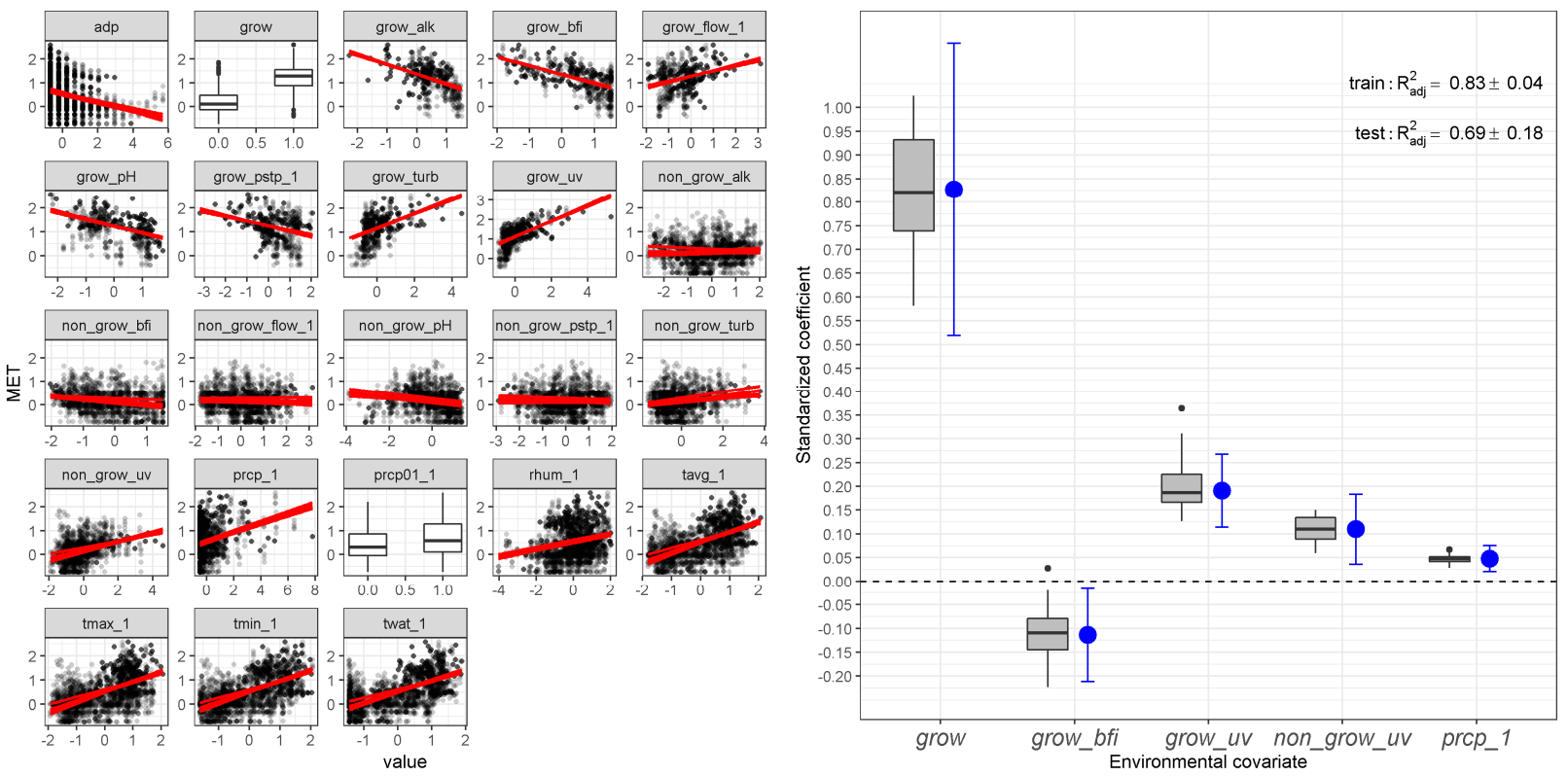

Figure S48: Bivariate GLS regression plots for MET (left) and significant final standardized coefficients (right).
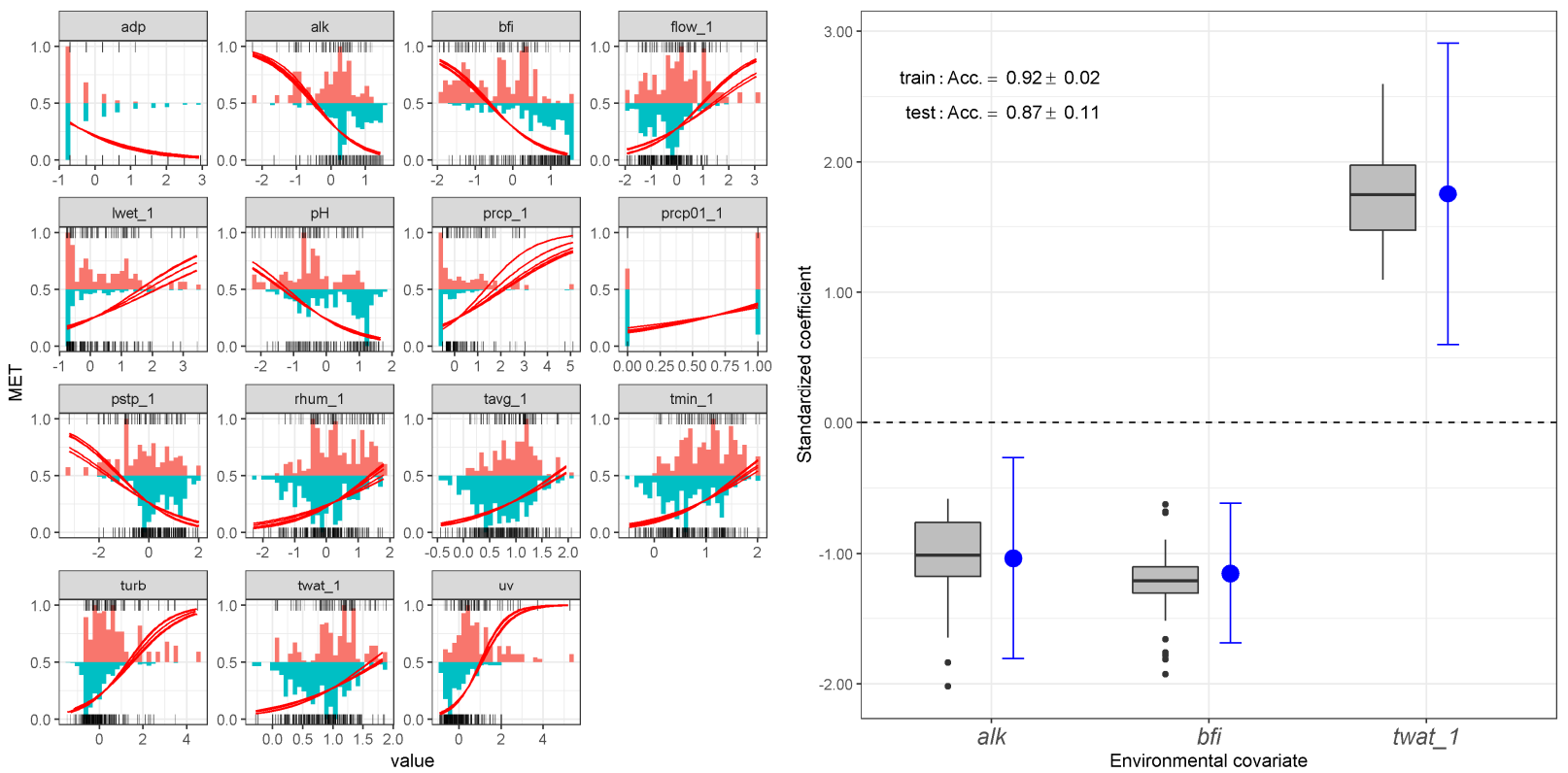

Figure S49: Bivariate logit regression plots for MET (left) and significant final standardized coefficients (right) during the growing season. 
Metolachlor-OXA (MET.o)
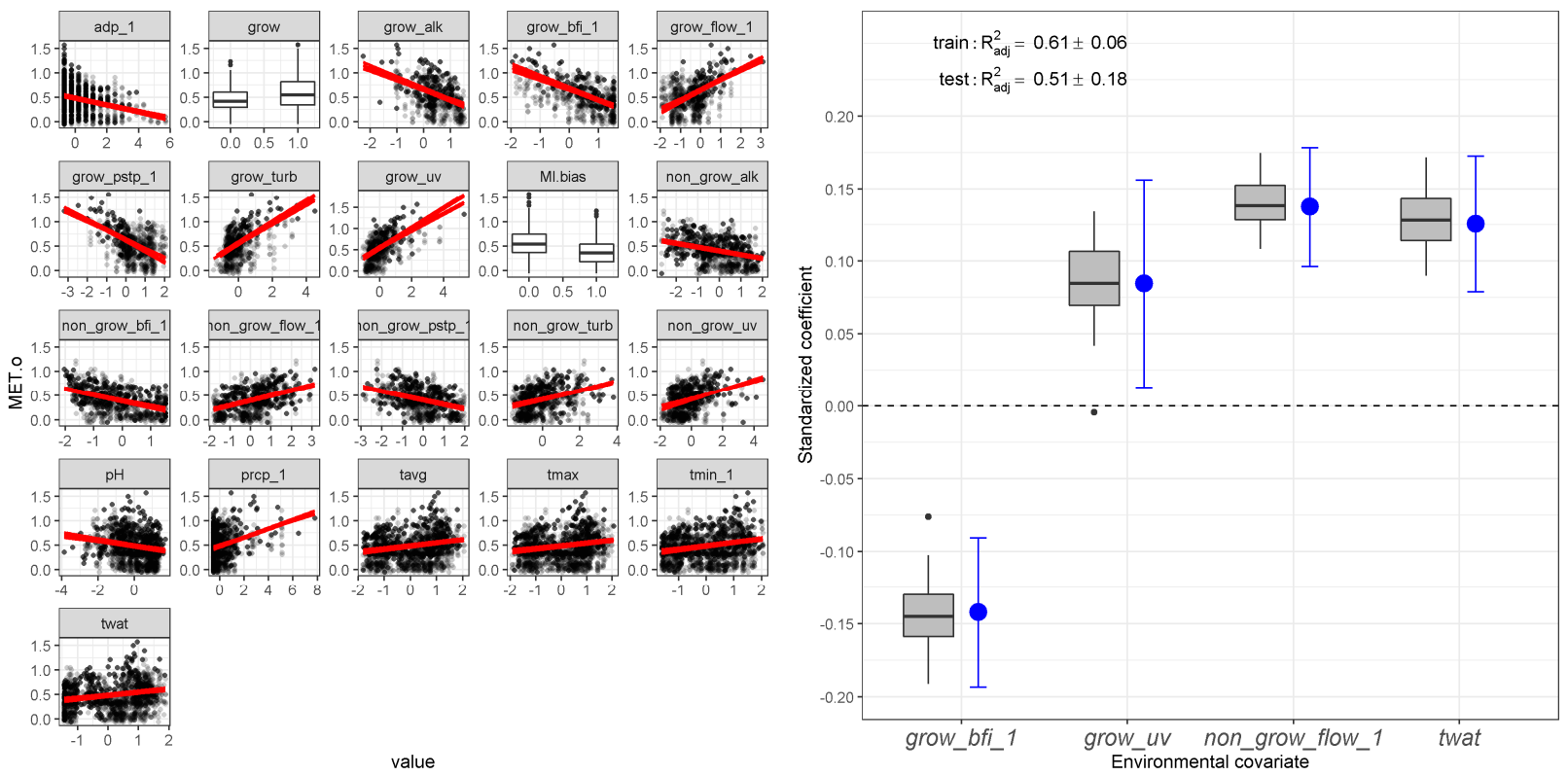

Figure S50: Bivariate GLS regression plots for MET.o (left) and significant final standardized coefficients (right).
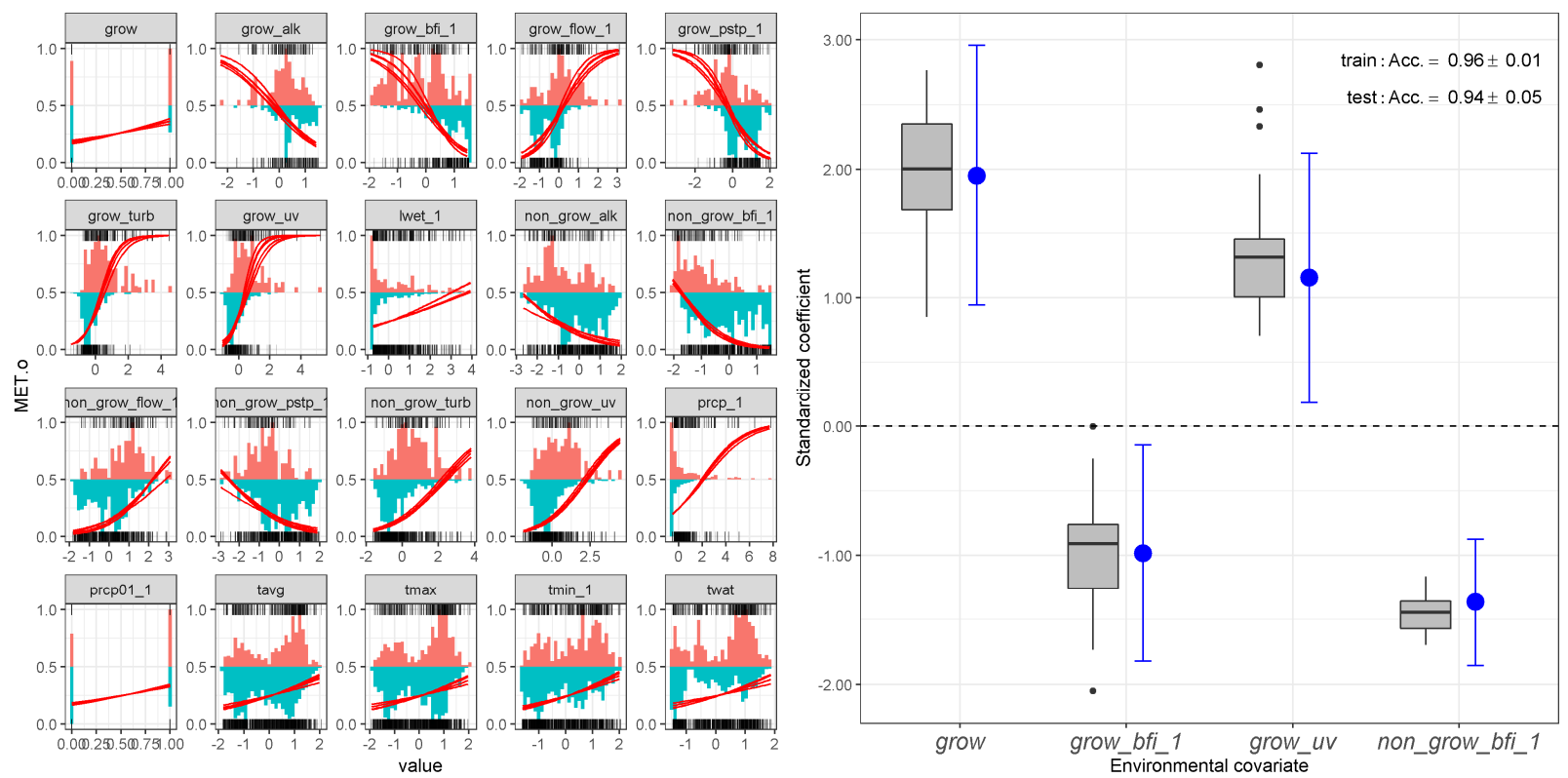

Figure S51: Bivariate logit regression plots for MET.o (left) and significant final standardized coefficients (right). 


\section{Desvenlafaxine (DES)}
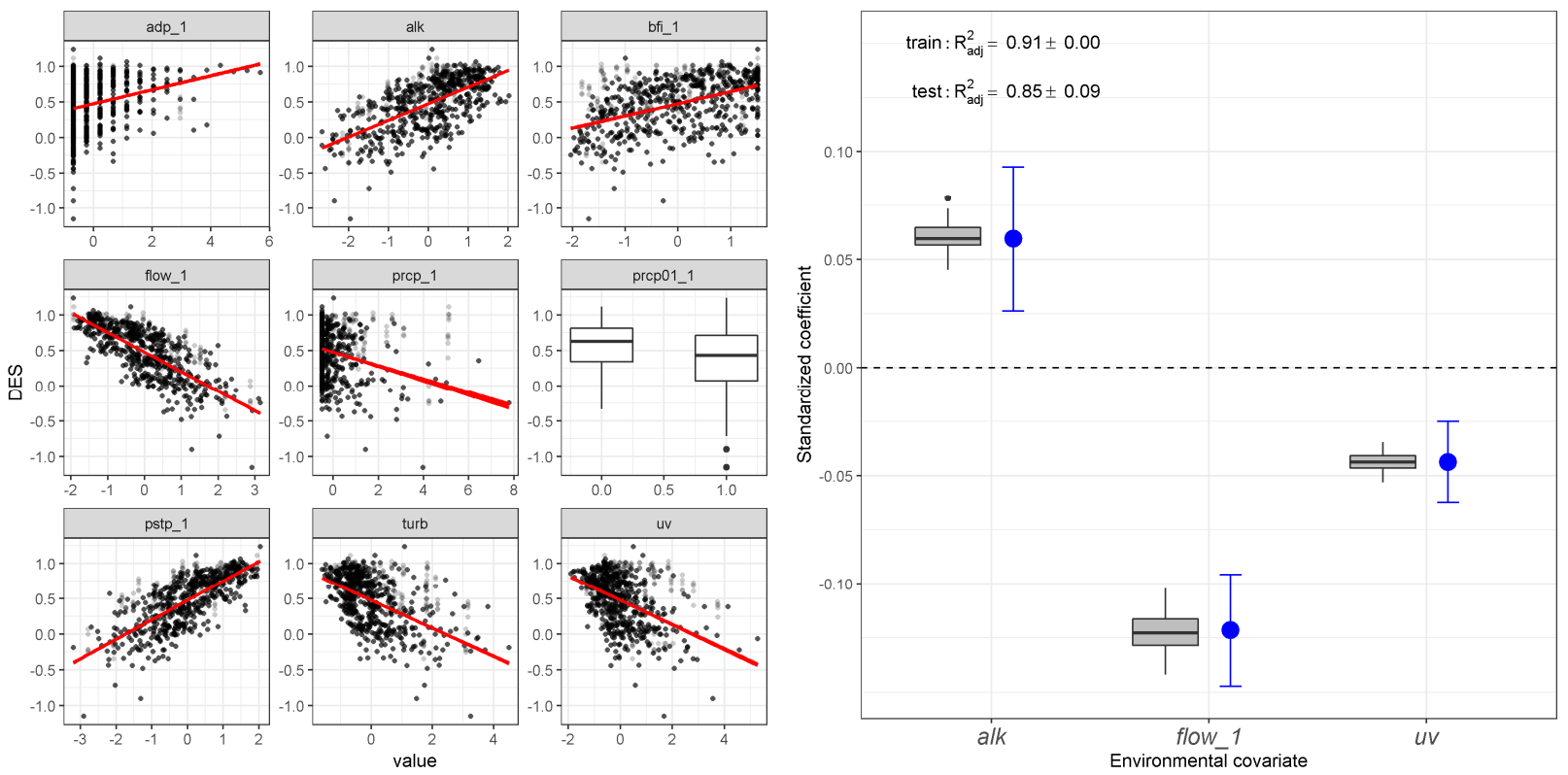

Figure S52: Bivariate GLS regression plots for DES (left) and significant final standardized coefficients (right).
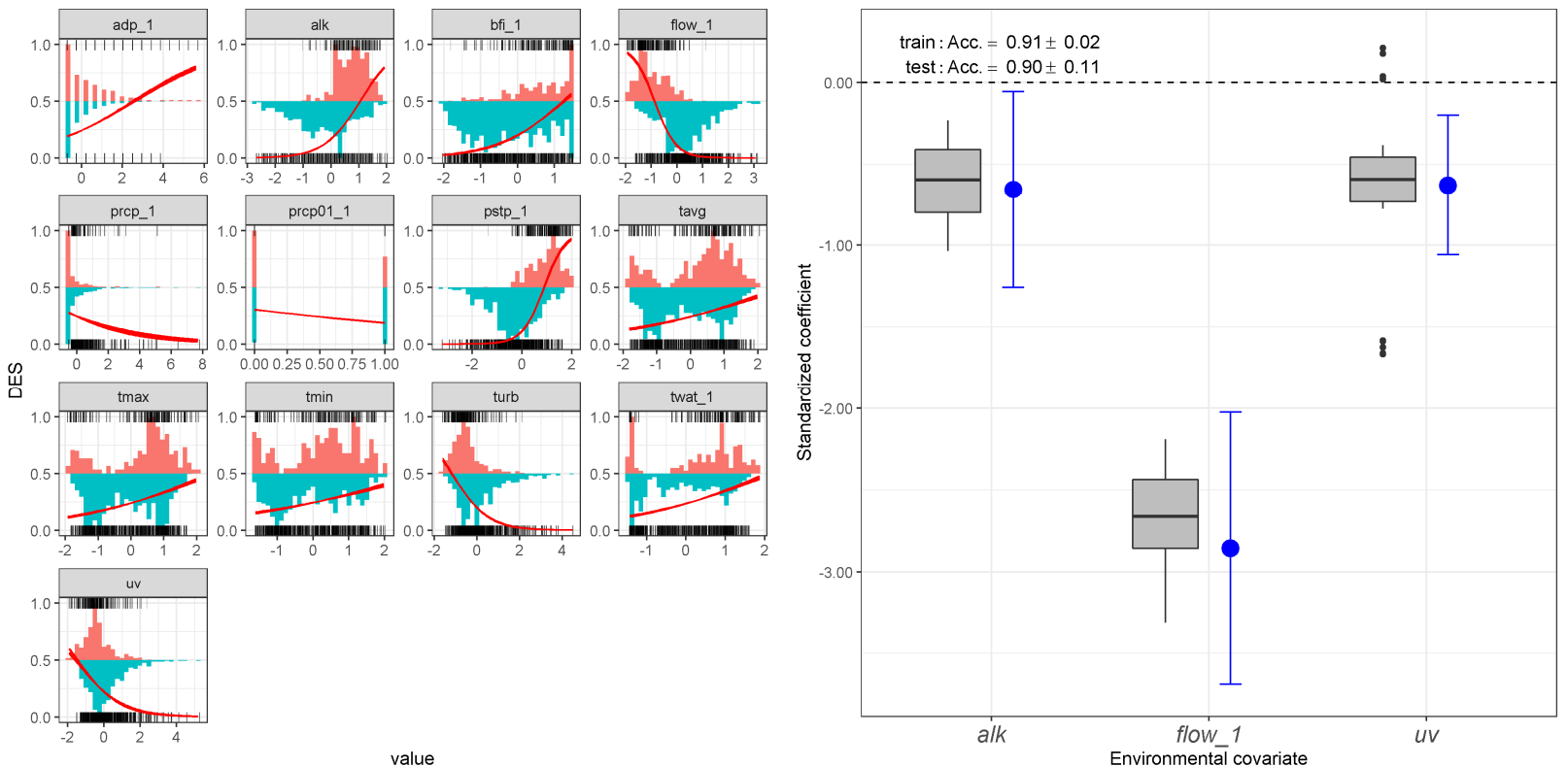

Figure S53: Bivariate logit regression plots for DES (left) and significant final standardized coefficients (right). 


\section{Rac-threo-dihydrobupropion (DHB)}
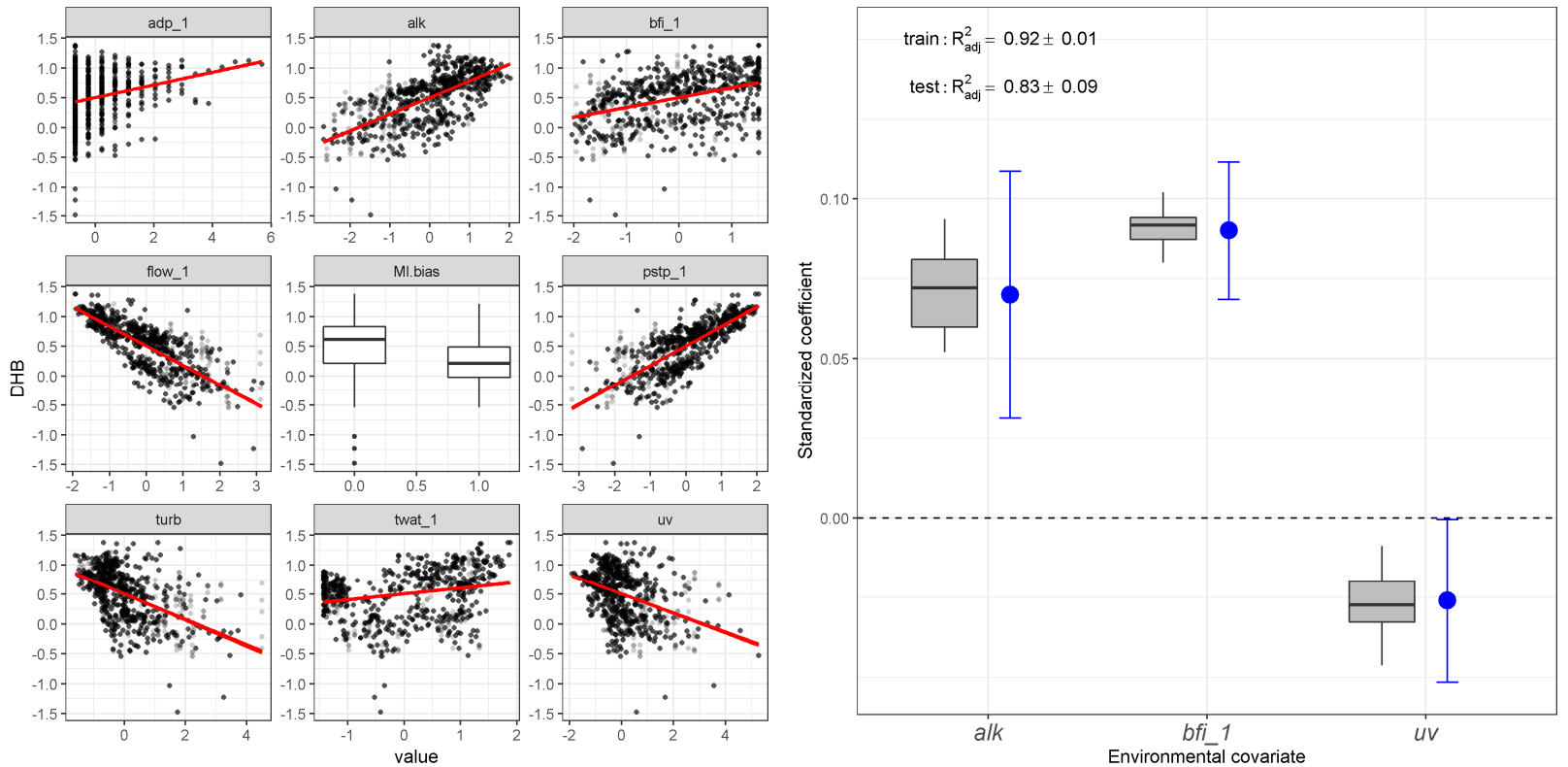

Figure S54: Bivariate GLS regression plots for DHB (left) and significant final standardized coefficients (right).
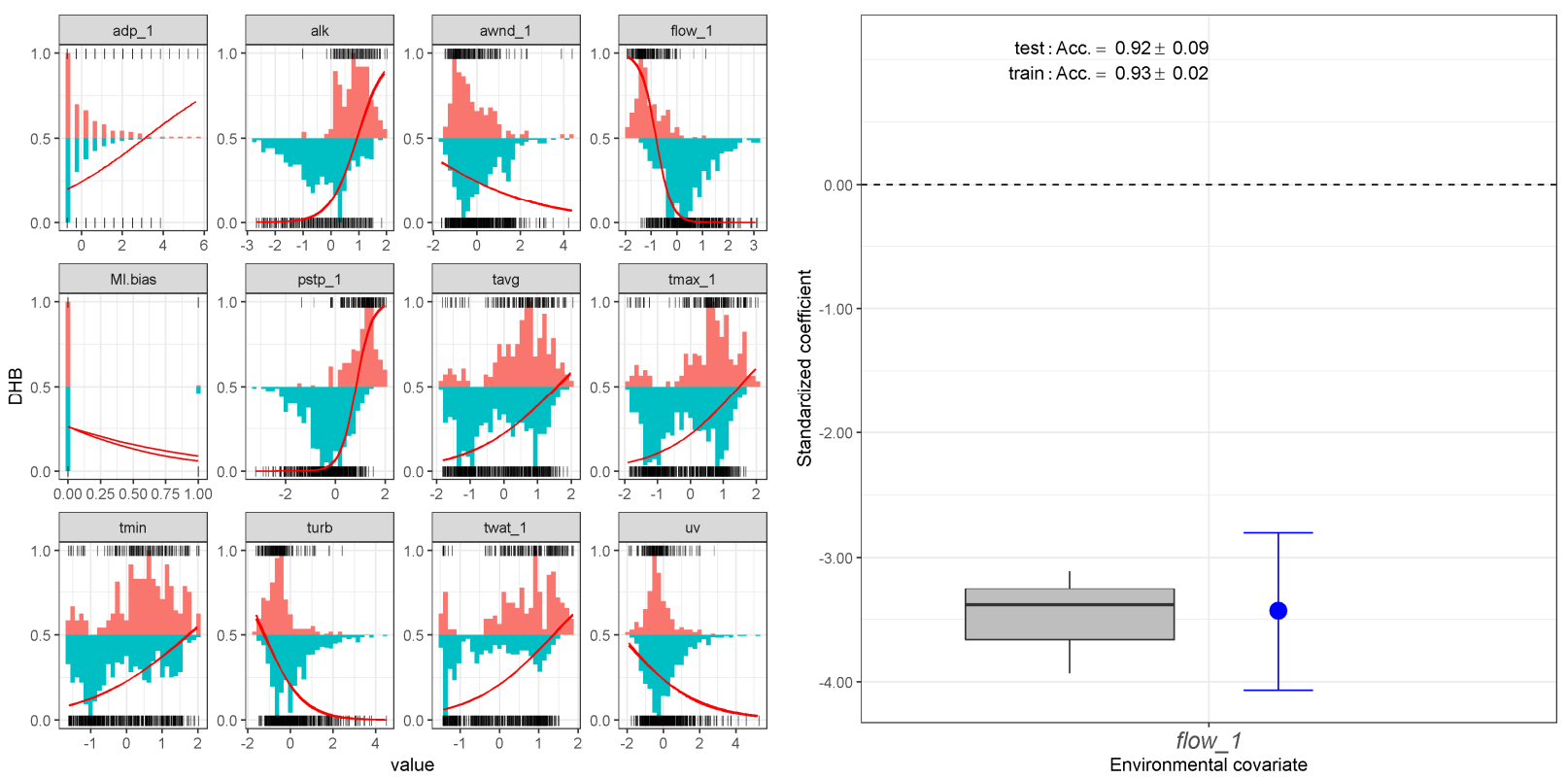

Figure S55: Bivariate logit regression plots for DHB (left) and significant final standardized coefficients (right). 


\section{Gabapentin-lactam (GAB.l)}
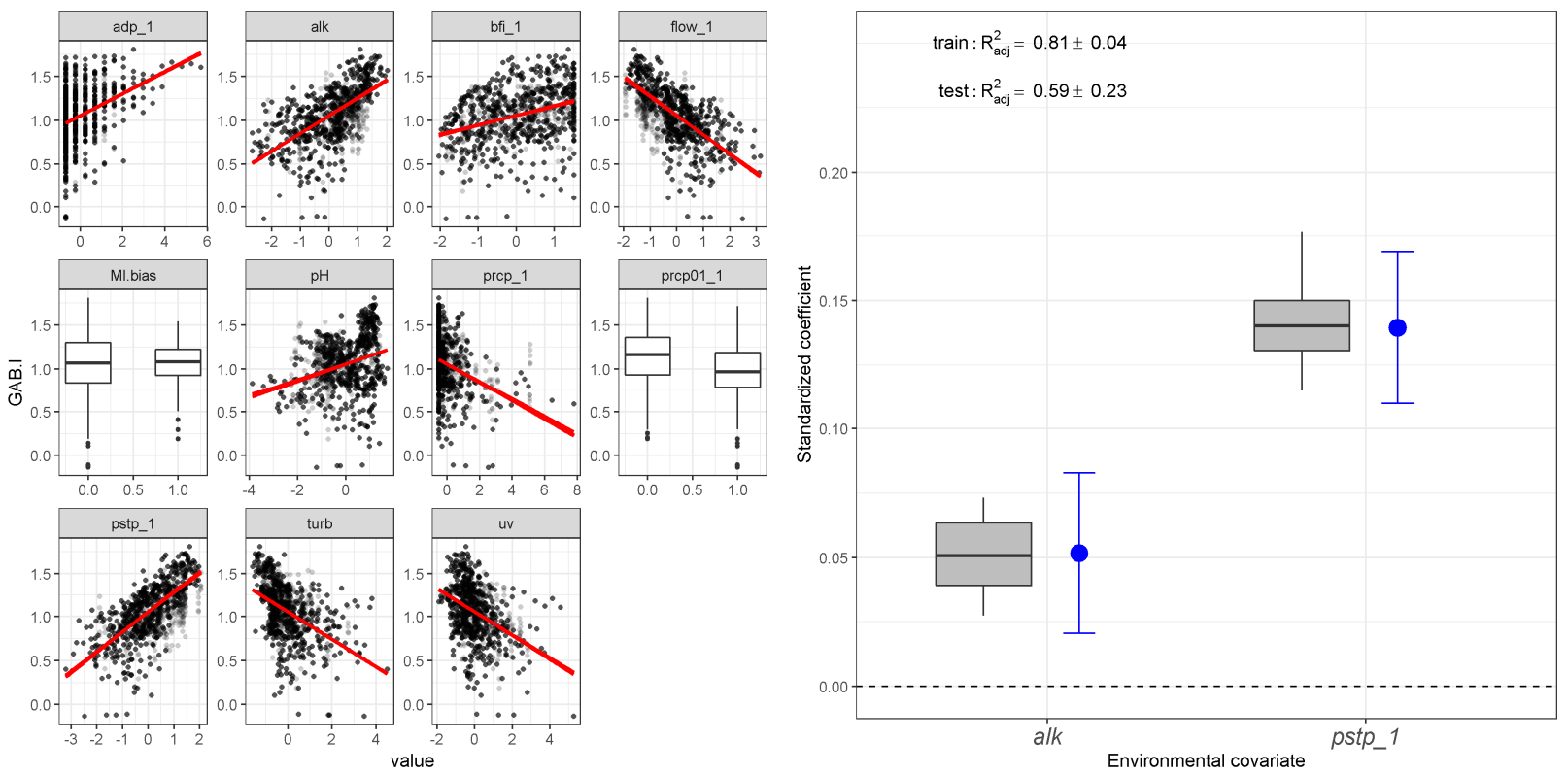

Figure S56: Bivariate GLS regression plots for GAB.l (left) and significant final standardized coefficients (right).
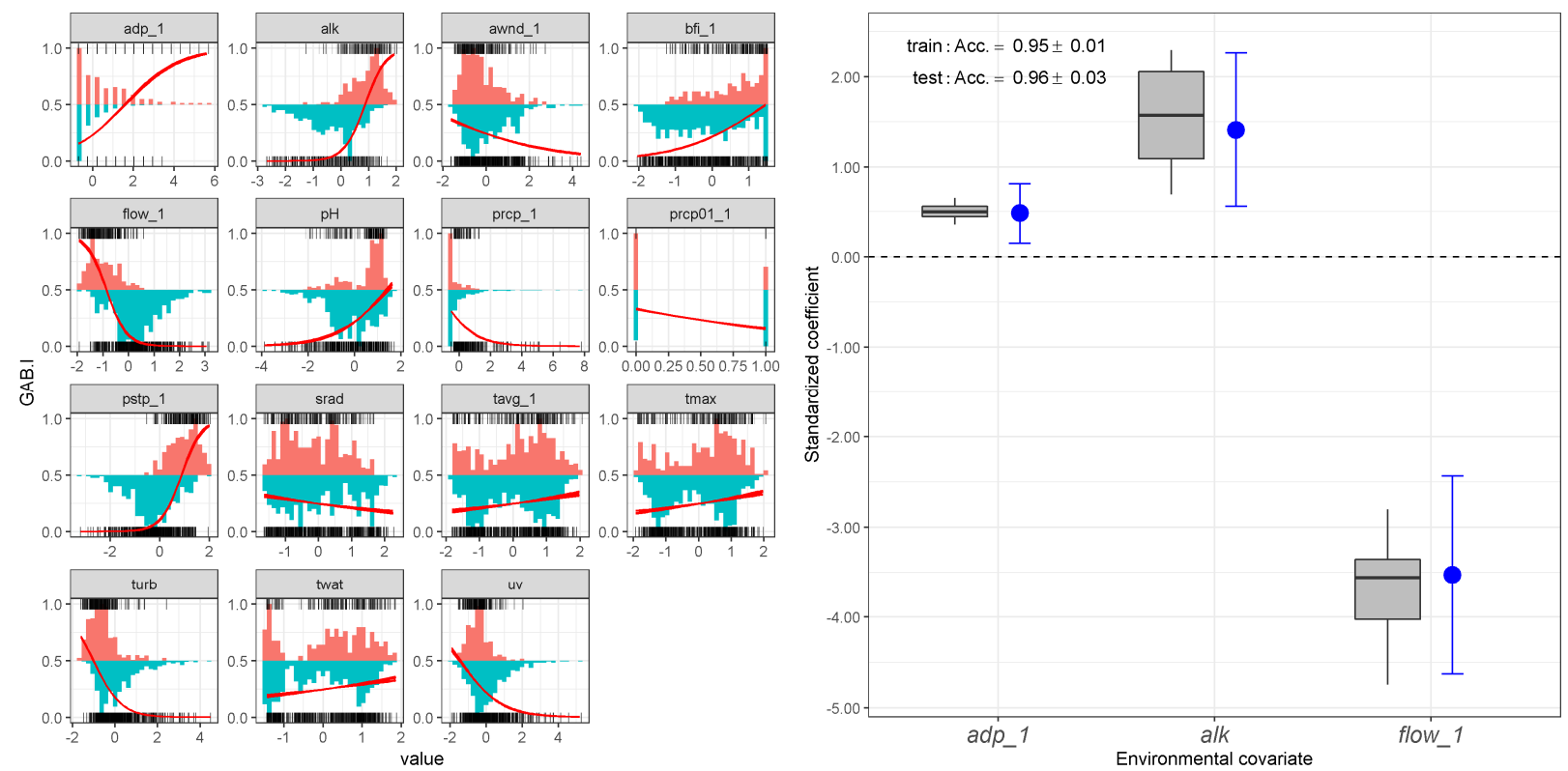

Figure S57: Bivariate logit regression plots for GAB.l (left) and significant final standardized coefficients (right). 
Cumulative normalized abundance (CUMA)
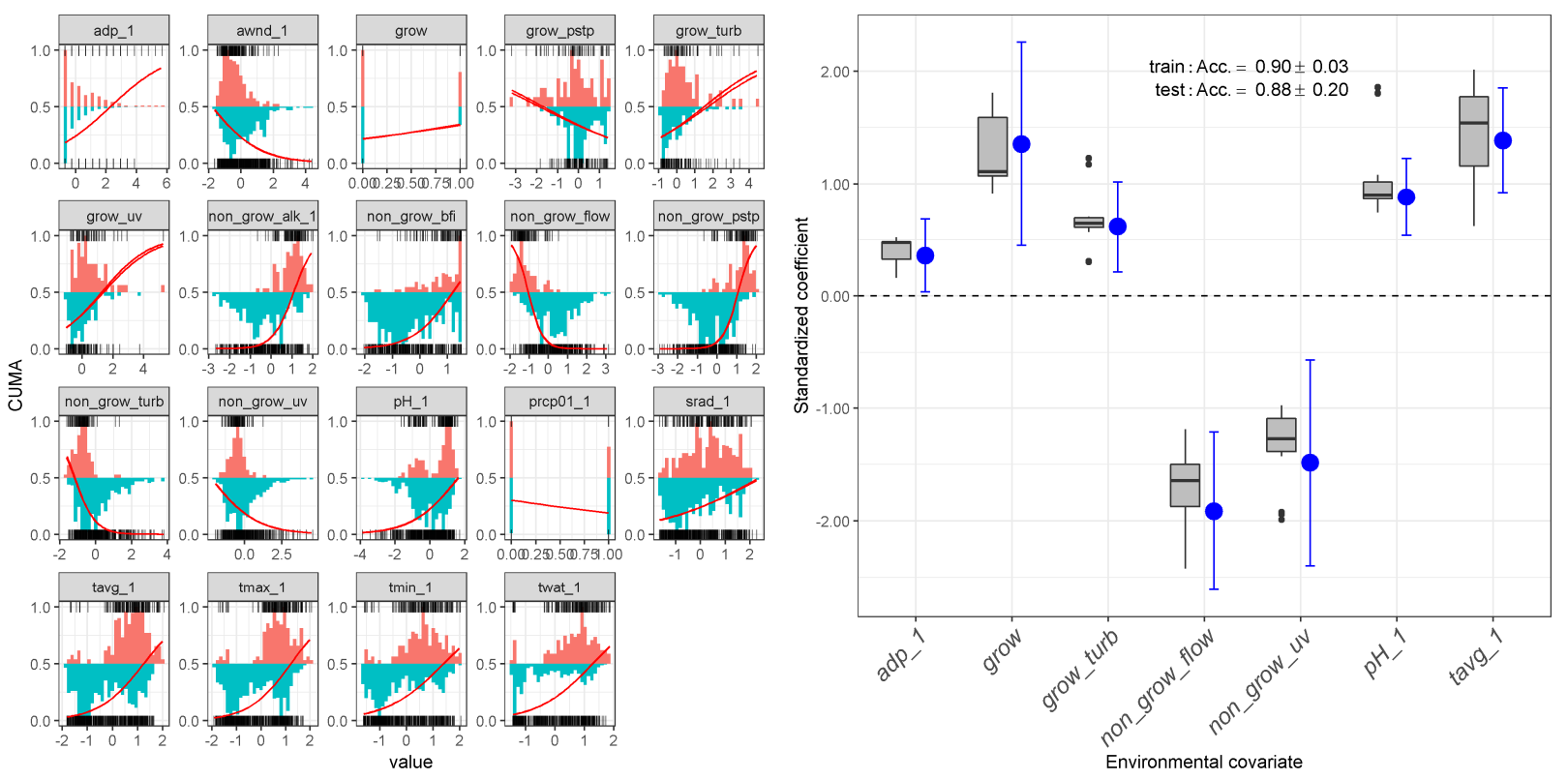

Figure S58: Bivariate logit regression plots for CUMA (left) and significant final standardized coefficients (right). 


\section{Cumulative estimated concentration (CUMC)}
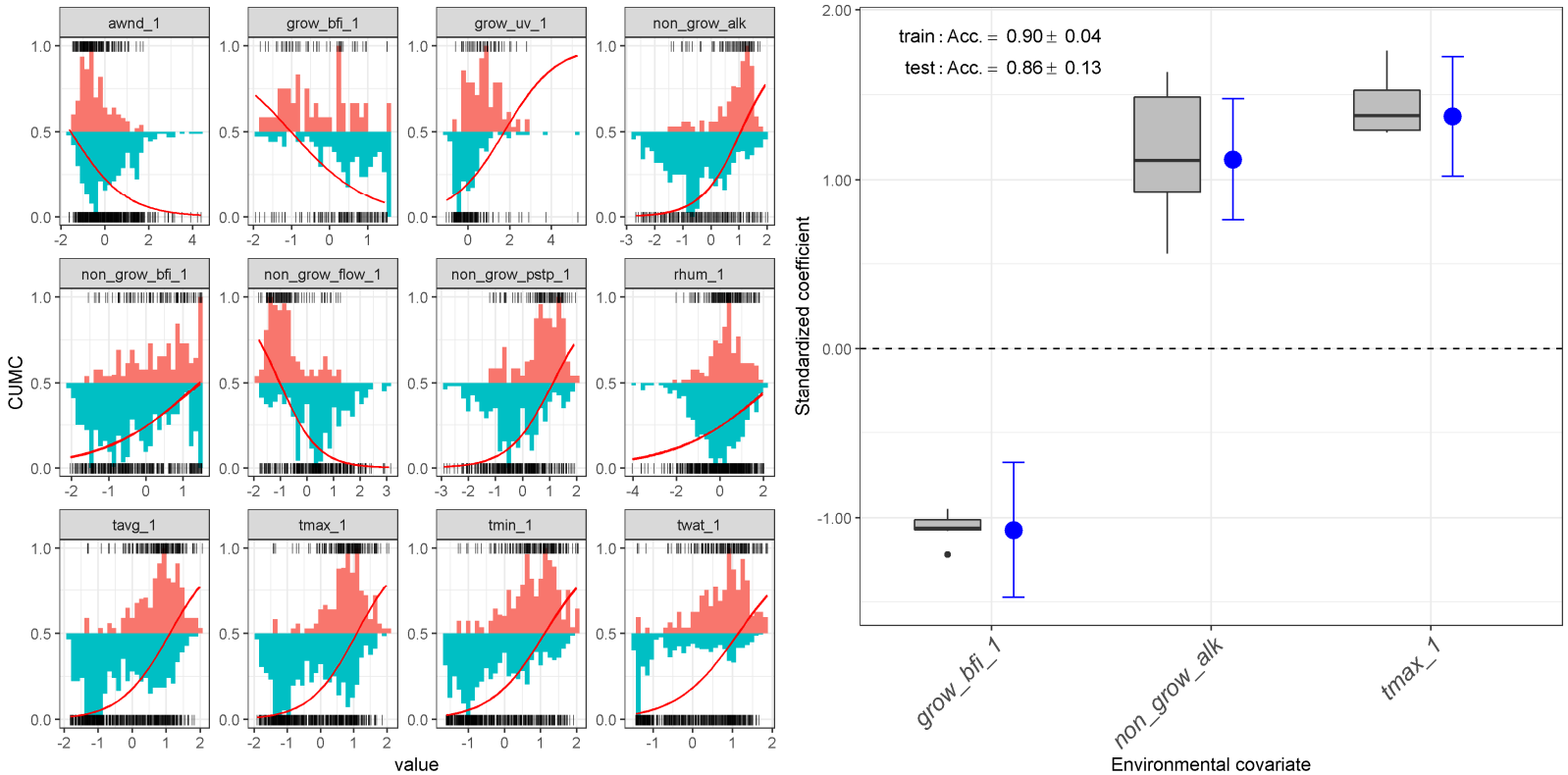

Figure S59: Bivariate logit regression plots for CUMC (left) and significant final standardized coefficients (right).
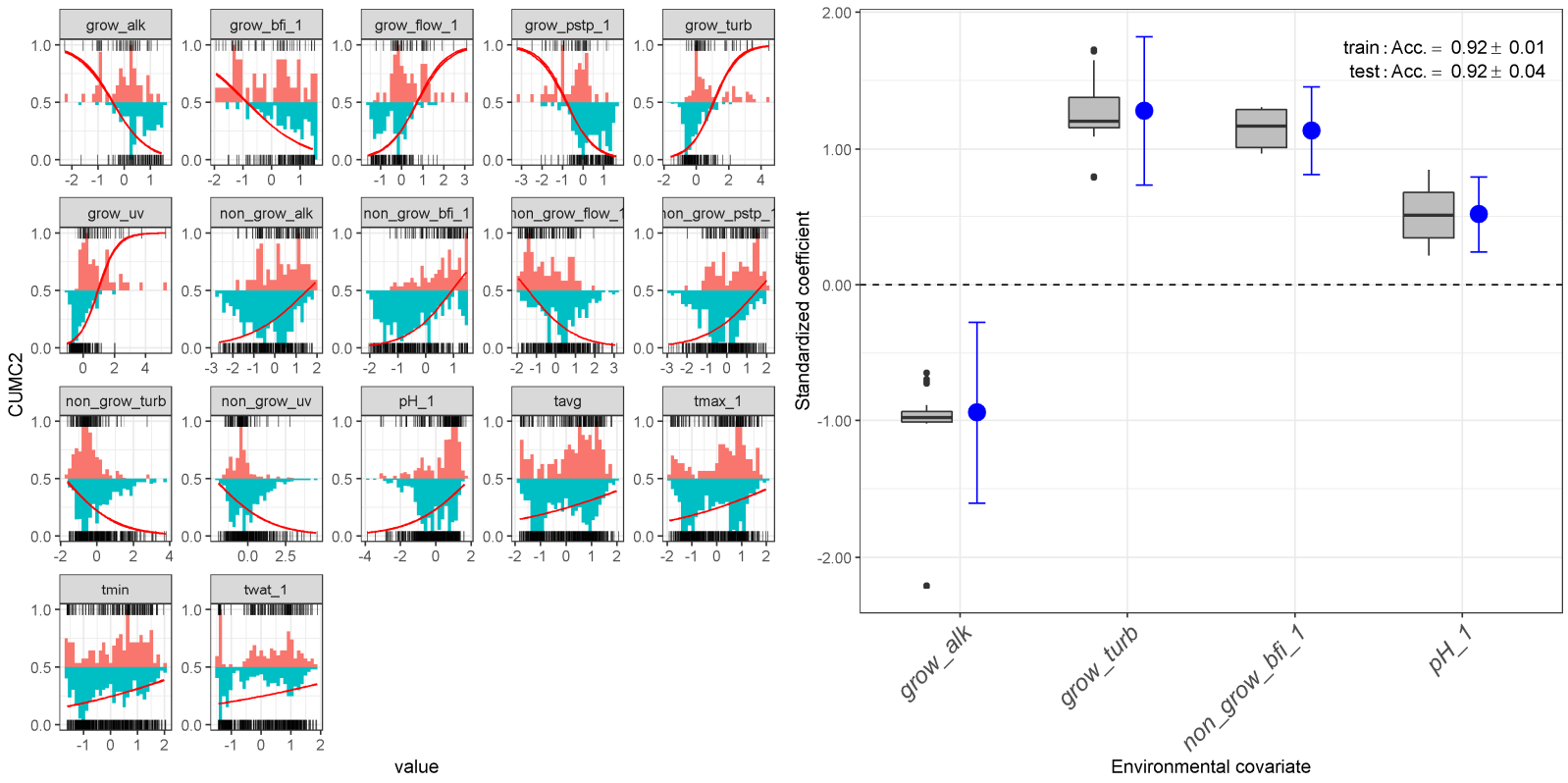

Figure S60: Bivariate logit regression plots for CUMC2 (left) and significant final standardized coefficients (right). 
Cumulative estimated risk (CUMT)
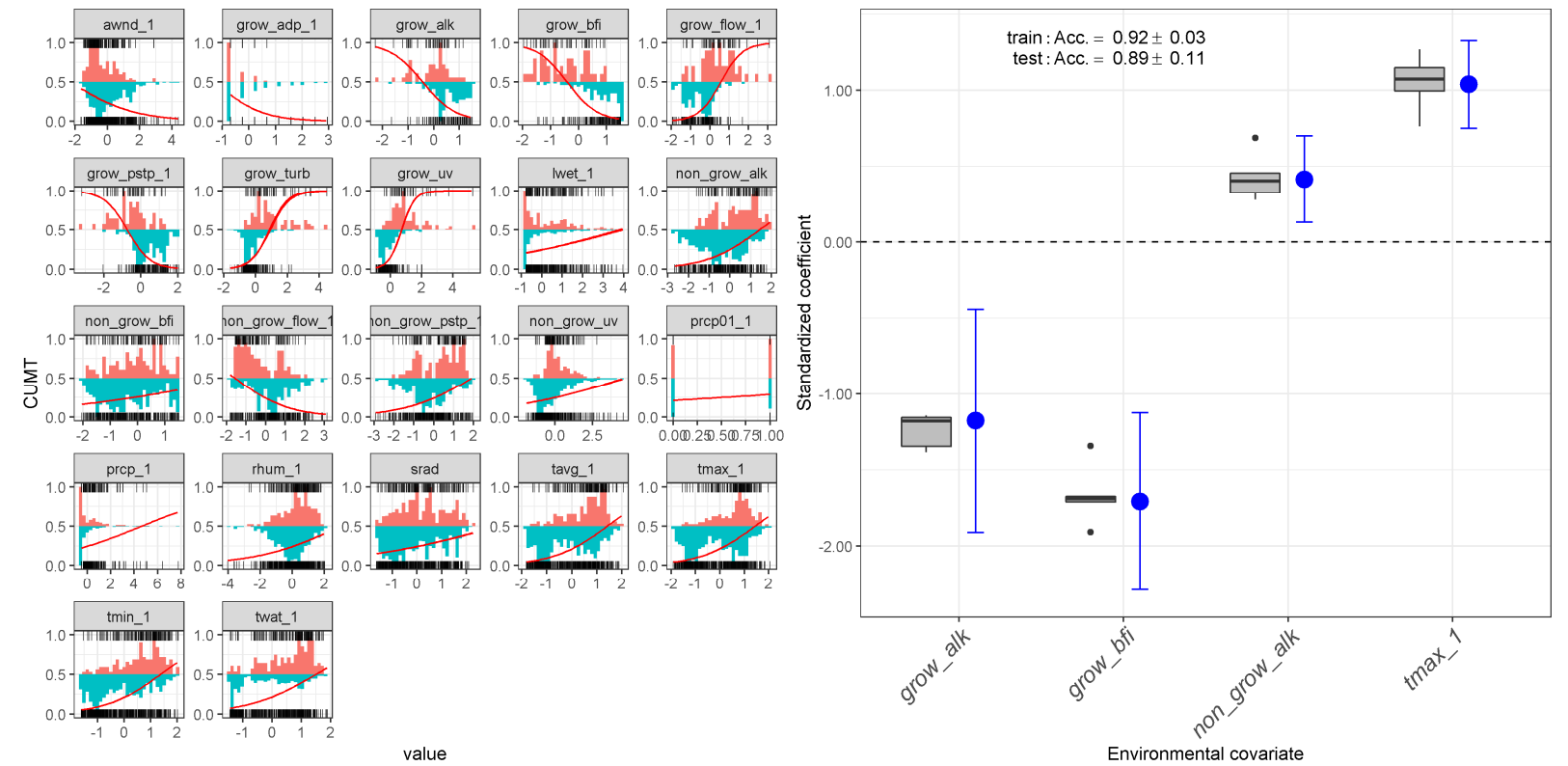

Figure S61: Bivariate logit regression plots for CUMT (left) and significant final standardized coefficients (right). 


\section{Final results}

Table S1: Regression results using the final averaged coefficients for micropollutant dynamics and peak events of representative micropollutant profiles. Significant covariates are listed for each micropollutant profile with the sign of the relationship (+/-). Final model diagnostics $\left(\mathrm{R}^{2}\right.$ and area under curve, AUC) represent each imputed dataset (average \pm s.d.).

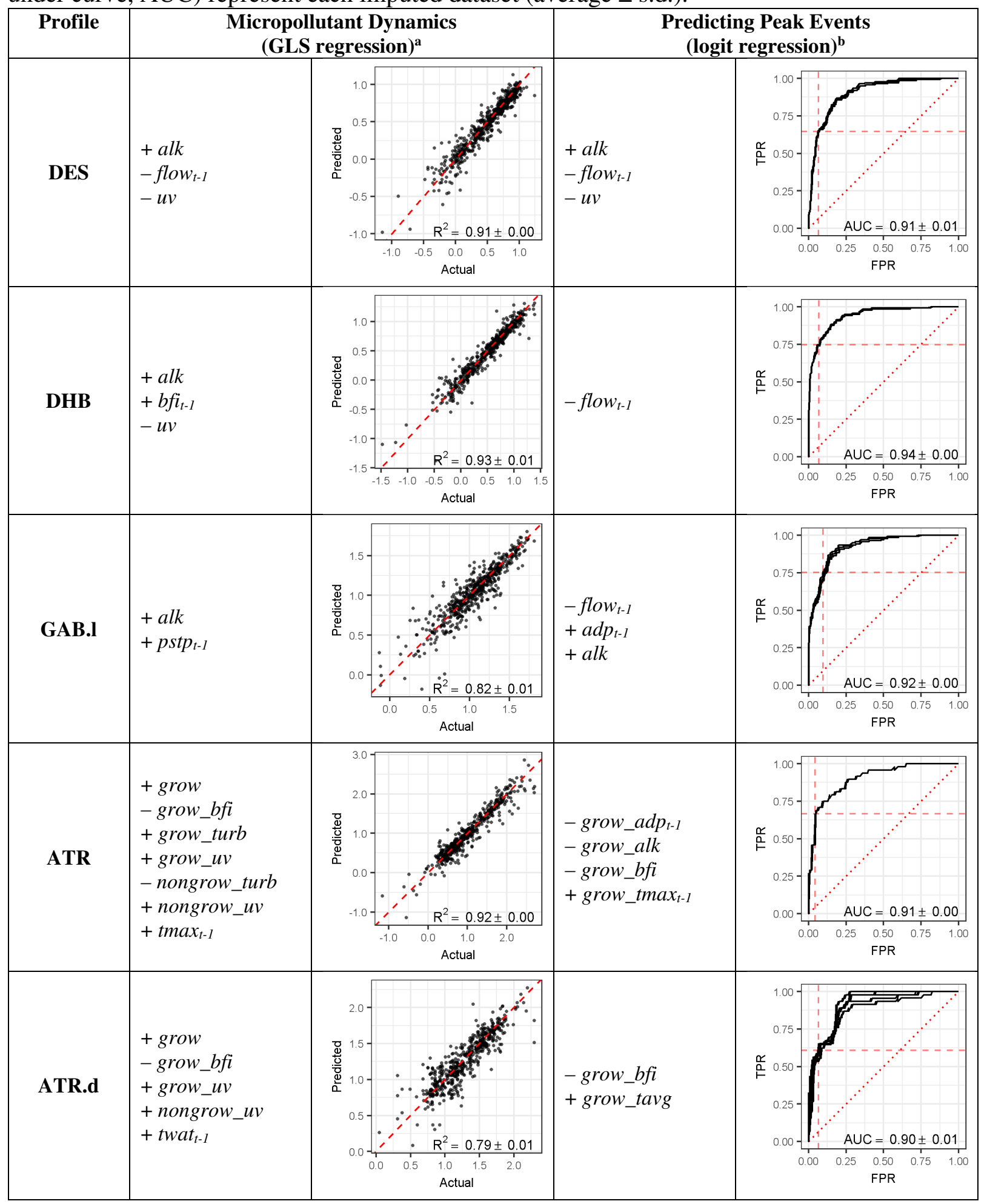




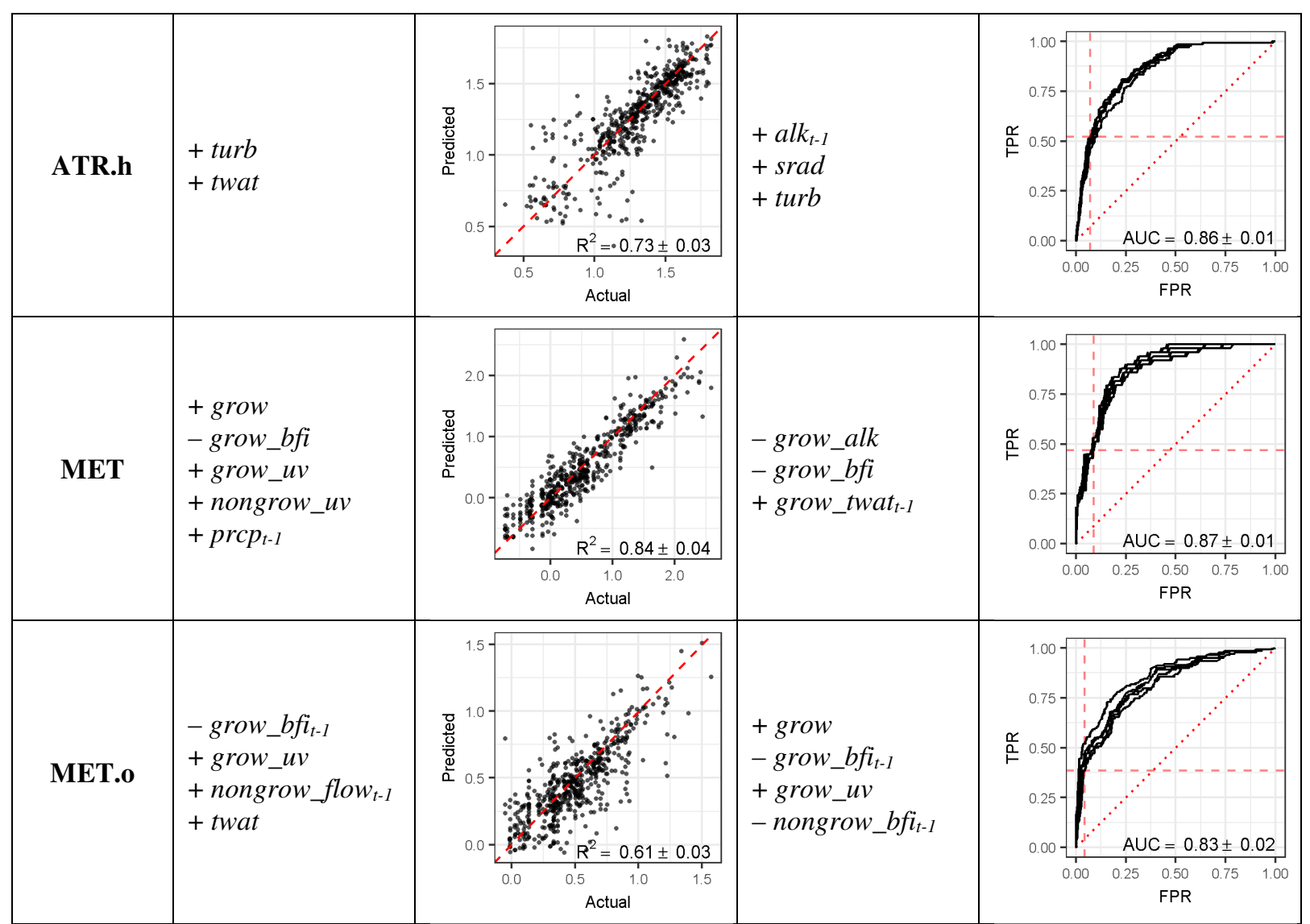

${ }^{a}$ GLS regression diagnostic plots represent the actual profile values vs. predicted values using final regression coefficients; dashed line $=1: 1$.

${ }^{\mathrm{b}}$ Logit regression diagnostic plots represent receiver operating characteristic (ROC) curves; $\mathrm{FPR}=$ false positive rate $(1-$ specificity); TPR $=$ true positive rate (sensitivity); dashed line $=$ cutoff at probability of 0.5 ; dotted line $=$ random guess, $1: 1$. 
Table S2: Regression results using the final averaged coefficients for peak events of cumulative profiles. Significant covariates are listed for each micropollutant profile with the sign of the relationship (+/-). Final model diagnostics (area under curve, AUC) represent each imputed dataset (average \pm s.d.).

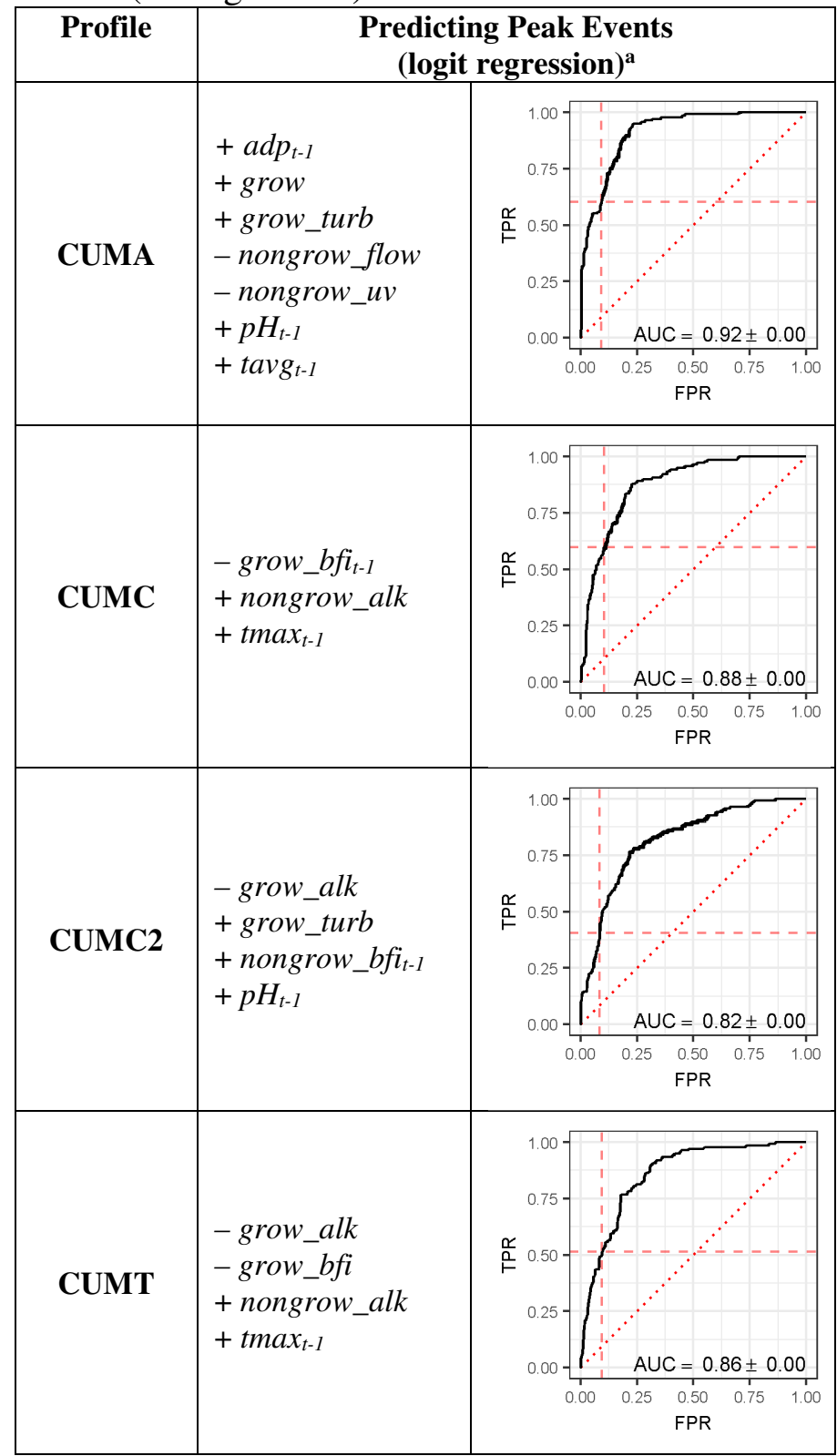

${ }^{a}$ Logit regression diagnostic plots represent receiver operating characteristic (ROC) curves; FPR $=$ false positive rate $(1-$ specificity); TPR $=$ true positive rate (sensitivity); dashed line $=$ cutoff at probability of 0.5 ; dotted line $=$ random guess, $1: 1$. 


\section{Recommendations for future micropollutant sampling strategies}

Table S3: Percent of environmental risk captured using uninformed and informed micropollutant sampling strategies. Informed strategies included the final peak event model and important bivariate models. The percent of environmental risk captured by each sampling strategy was determined by comparing the areas under the resulting profiles with the areas under the actual representation of risk profiles.

\begin{tabular}{|c|c|c|c|c|c|c|c|c|c|}
\hline \multicolumn{2}{|c|}{ Profile } & \multirow{3}{*}{$\begin{array}{c}\text { Uninformed }^{a} \\
53 \pm 10 \%\end{array}$} & \multicolumn{7}{|c|}{ Informed } \\
\hline & & & final & $b f i$ & flow & alk & turb & $u v$ & prcp \\
\hline \multirow{2}{*}{ ATR } & weekly & & $99 \%$ & $92 \%$ & $92 \%$ & $98 \%$ & $75 \%$ & $95 \%$ & $76 \%$ \\
\hline & monthly & $29 \pm 8 \%$ & $106 \%$ & $92 \%$ & $92 \%$ & $84 \%$ & $72 \%$ & $100 \%$ & $40 \%$ \\
\hline \multirow{2}{*}{ DES } & weekly & $82 \pm 3 \%$ & $87 \%$ & $88 \%$ & $84 \%$ & $86 \%$ & $87 \%$ & $86 \%$ & n.a. \\
\hline & monthly & $61 \pm 4 \%$ & $75 \%$ & $77 \%$ & $82 \%$ & $73 \%$ & $74 \%$ & $72 \%$ & n.a. \\
\hline \multirow{2}{*}{ CUMC } & weekly & $81 \pm 3 \%$ & $91 \%$ & $84 \%$ & $84 \%$ & $84 \%$ & $82 \%$ & $83 \%$ & $84 \%$ \\
\hline & monthly & $64 \pm 4 \%$ & $70 \%$ & $73 \%$ & $69 \%$ & $66 \%$ & $67 \%$ & $69 \%$ & $70 \%$ \\
\hline \multirow{2}{*}{ CUMC2 } & weekly & $69 \pm 4 \%$ & $83 \%$ & $81 \%$ & $83 \%$ & $86 \%$ & $77 \%$ & $81 \%$ & $73 \%$ \\
\hline & monthly & $45 \pm 4 \%$ & $65 \%$ & $67 \%$ & $69 \%$ & $65 \%$ & $63 \%$ & $77 \%$ & $48 \%$ \\
\hline \multirow{2}{*}{ CUMT } & weekly & $66 \pm 4 \%$ & $92 \%$ & $84 \%$ & $84 \%$ & $91 \%$ & $75 \%$ & $84 \%$ & $79 \%$ \\
\hline & monthly & $45 \pm 6 \%$ & $85 \%$ & $79 \%$ & $78 \%$ & $76 \%$ & $67 \%$ & $93 \%$ & $54 \%$ \\
\hline
\end{tabular}

${ }^{a}$ Values represent the mean \pm standard deviation of area ratios for each uninformed starting date $\left(\mathrm{n}_{\text {weekly }}=7 ; \mathrm{n}_{\text {monthly }}=30\right)$. 

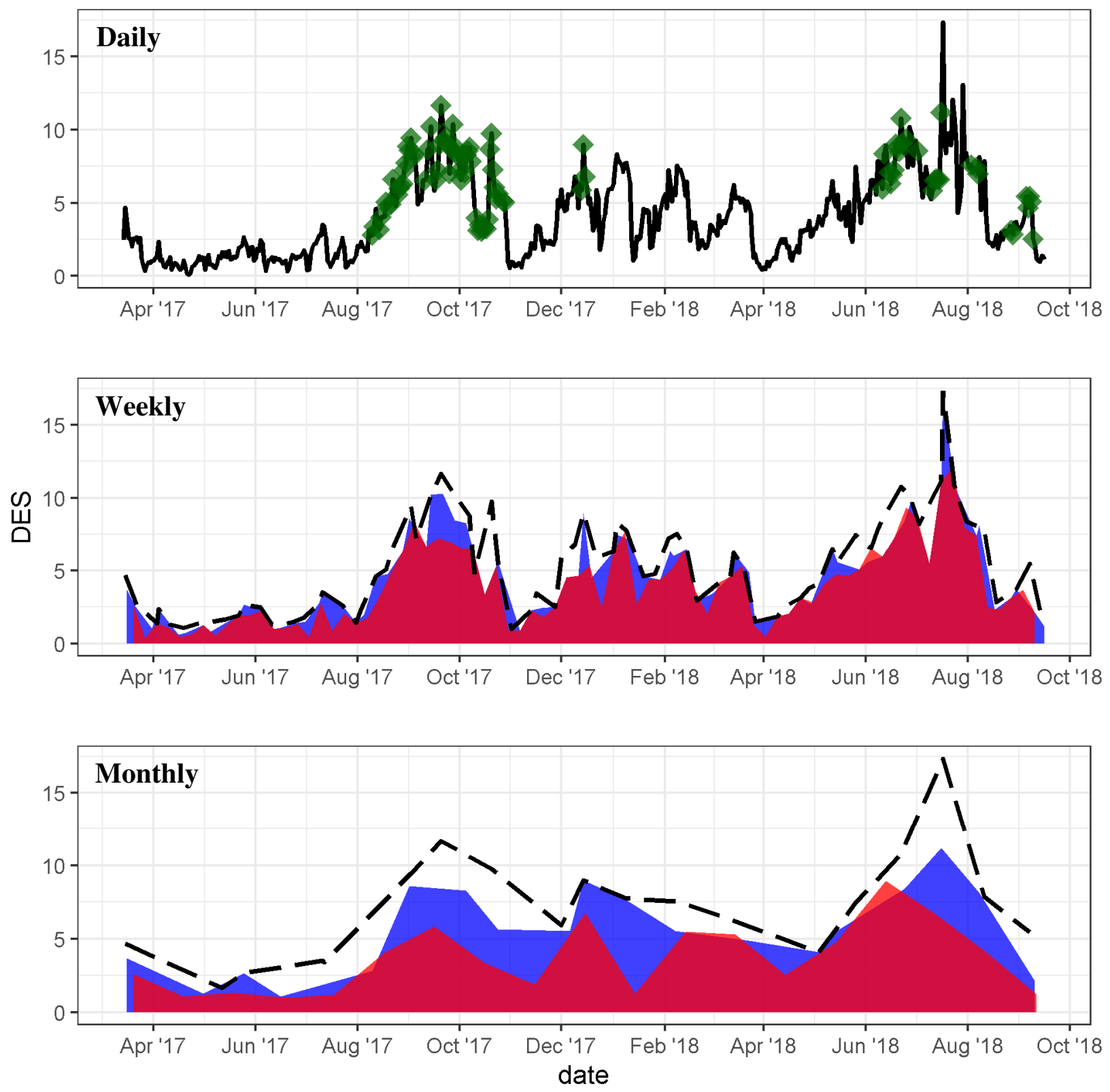

Figure S62: Informed vs uninformed sampling strategies for desvenlafaxine (DES) concentration in $n g \cdot \mathrm{L}^{-1}$; solid black line $=$ average of the imputed datasets; green points $=$ triggered sampling events $(\geq 2)$; dashed black line $=$ actual representation of risk; blue area $=$ informed sampling, red area $=$ uninformed sampling. 

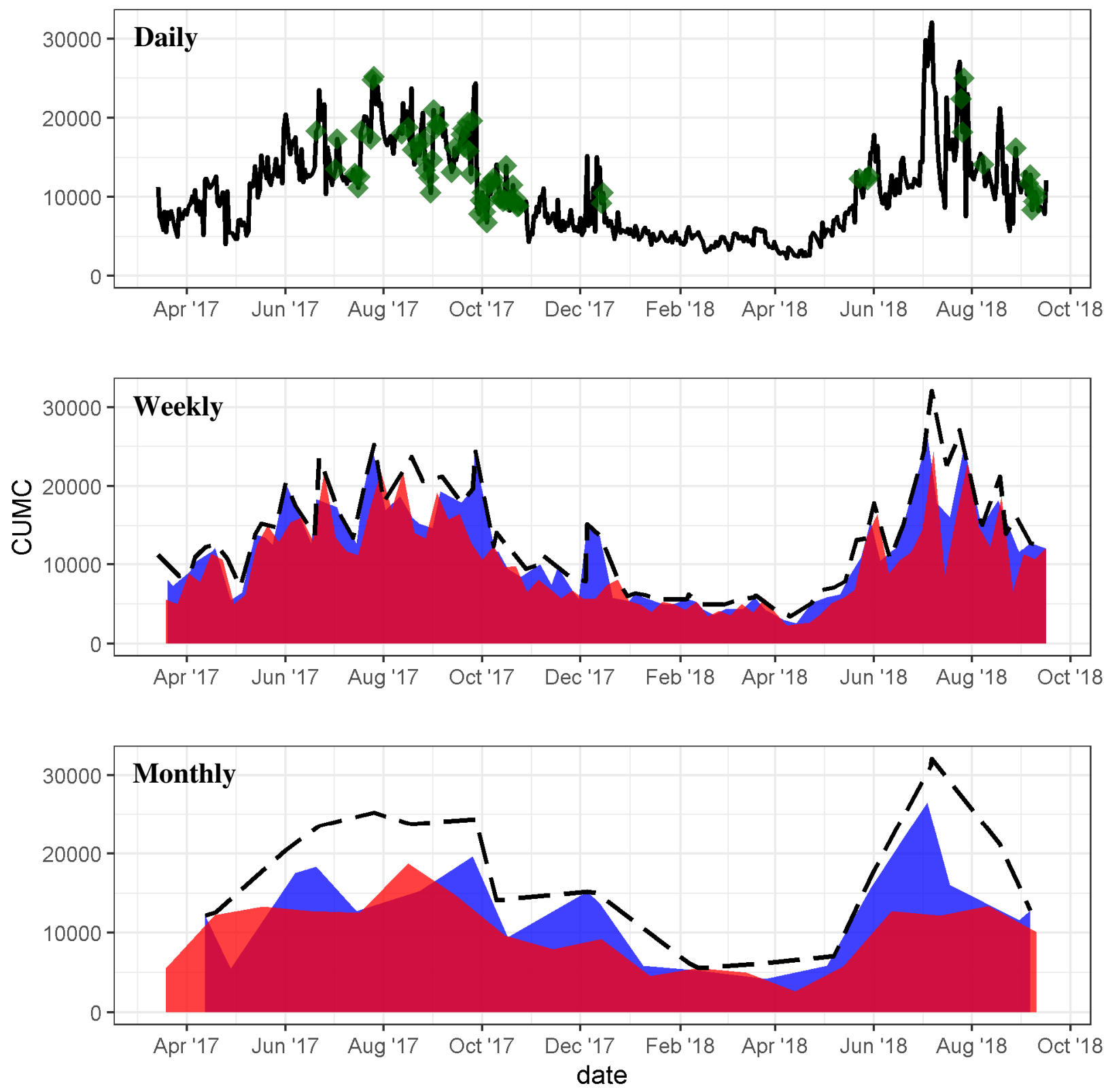

Figure S63: Informed vs uninformed sampling strategies for cumulative estimated concentration $(\mathrm{CUMC})$ in $\mathrm{ng} \cdot \mathrm{L}^{-1}$; solid black line = average of the imputed datasets; green points = triggered sampling events $(\geq 2)$; dashed black line $=$ actual representation of risk; blue area $=$ informed sampling, red area $=$ uninformed sampling. 

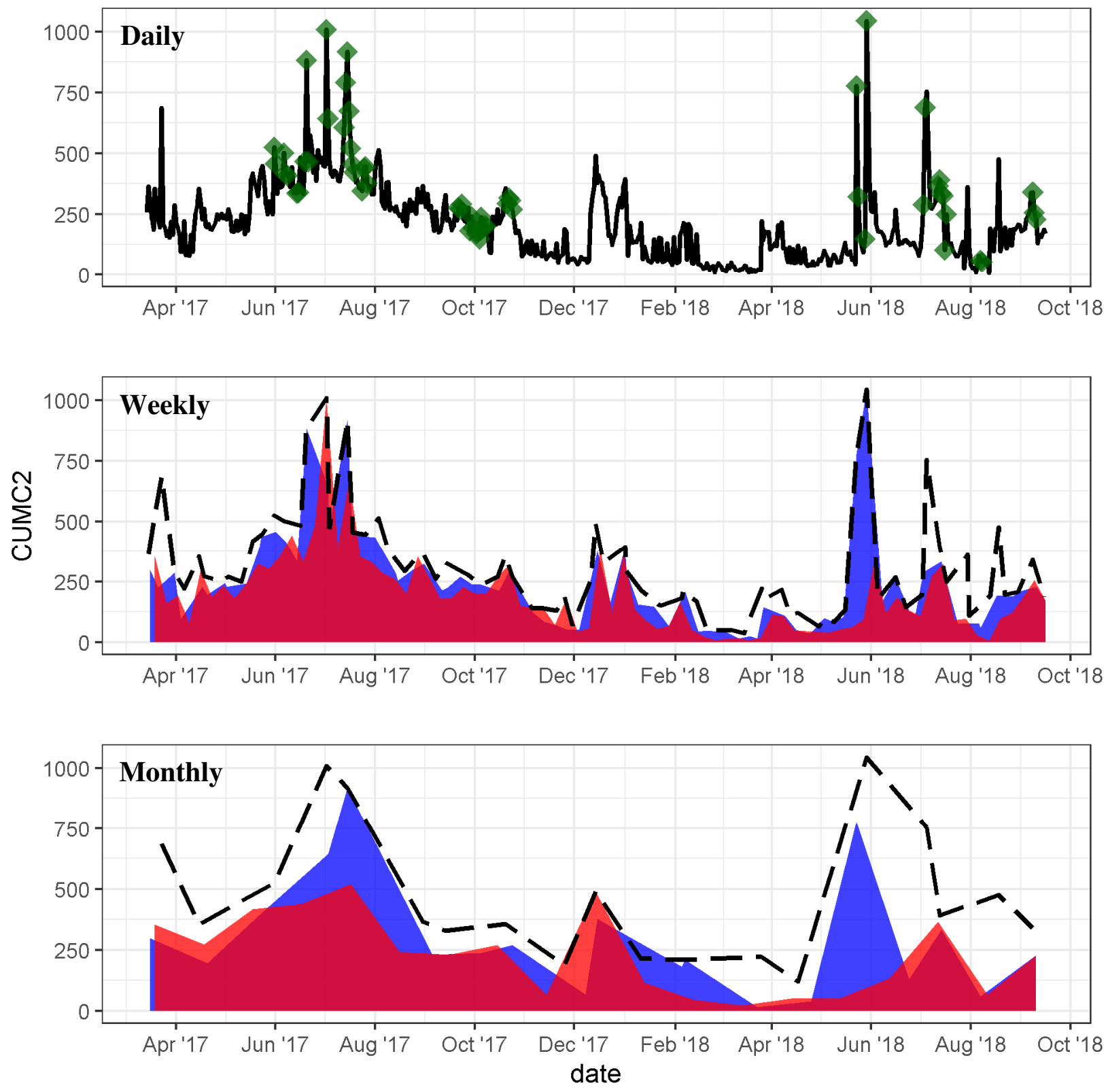

Figure S64: Informed vs uninformed sampling strategies for cumulative estimated concentration $\left(\right.$ CUMC2) in $n g \cdot \mathrm{L}^{-1}$; solid black line = average of the imputed datasets; green points = triggered sampling events $(\geq 2)$; dashed black line $=$ actual representation of risk; blue area $=$ informed sampling, red area $=$ uninformed sampling. 

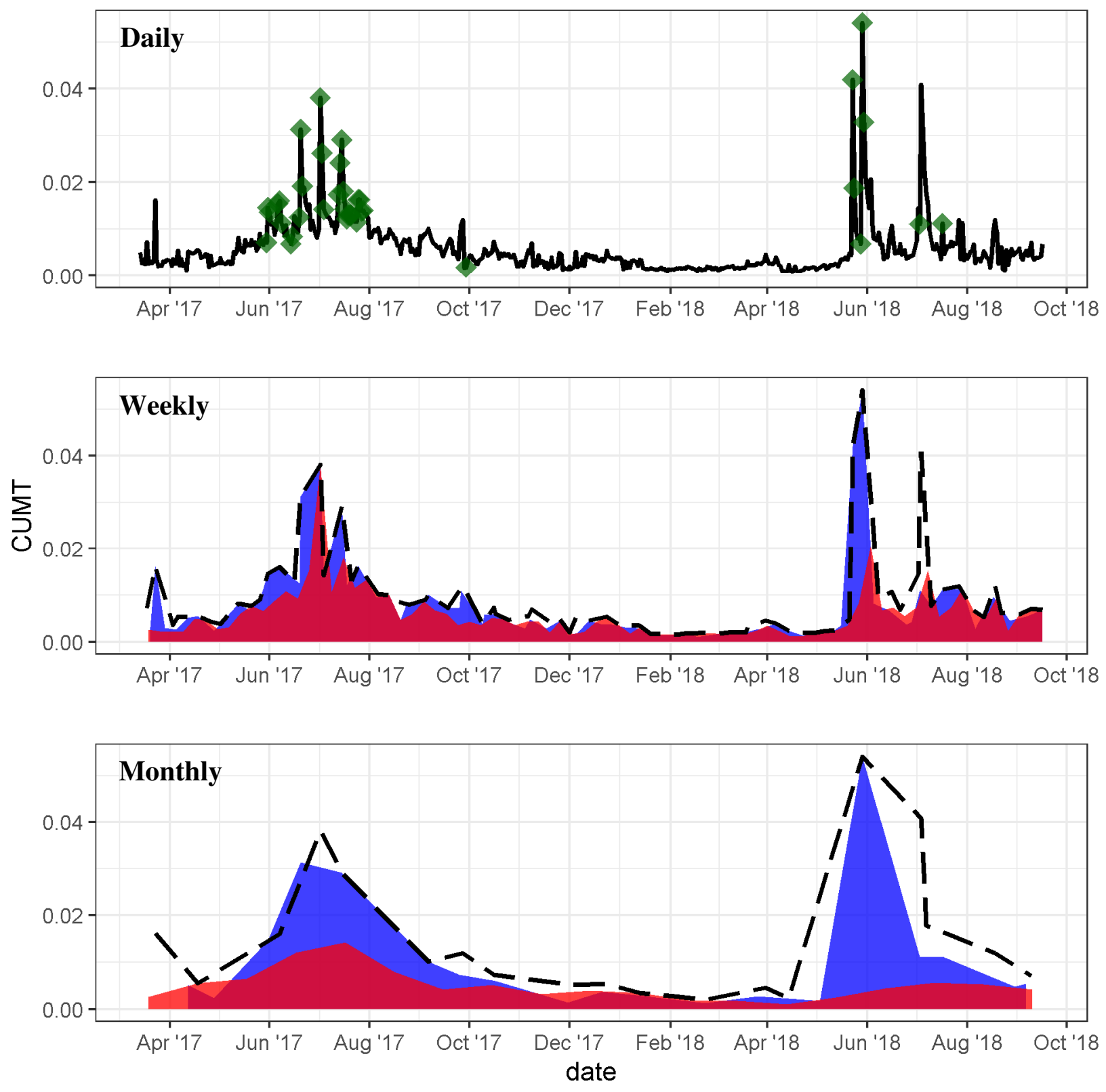

Figure S65: Informed vs uninformed sampling strategies for cumulative estimated risk (CUMT) expressed as exposure-activity ratio (EAR); solid black line = average of the imputed datasets; green points $=$ triggered sampling events $(\geq 2)$; dashed black line $=$ actual representation of risk; blue area $=$ informed sampling, red area $=$ uninformed sampling. 


\section{References}

(1) Menne, M. J.; Durre, I.; Korzeniewski, B.; McNeal, S.; Thomas, K.; Yin, X.; Anthony, S.;

Ray, R.; Vose, R. S.; E.Gleason, B.; et al. Global Historical Climatology Network - Daily (GHCN-Daily), Version 3. NOAA Natl. Clim. Data Cent. 2012.

(2) Chamberlain, S. rnoaa: "NOAA" Weather Data from R https://cran.rproject.org/package=rnoaa.

(3) NOAA - Northeast Regional Climate Center at Cornell University. Climate Data. 2018.

(4) Pebesma, E. J. Multivariable Geostatistics in S: The Gstat Package. Comput. Geosci. 2004, $30(7), 683-691$.

(5) Baddeley, A.; Rubak, E.; Turner, R. Spatial Point Patterns: Methodology and Applications with R; Chapman and Hall/CRC Press: London, 2015.

(6) U.S. Geological Survey. National Water Information System data www.waterdata.usgs.gov/nwis.

(7) Tallaksen, L. M.; Van Lanen, H. A. J. Hydrological Drought: Processes and Estimation Methods for Streamflow and Groundwater; 2004; Vol. 48. 